Institut für Kernphysik

Mesonic Contributions to the Spin and Flavor Structure of the Nucleon
J. Speth
A.W. Thomas 


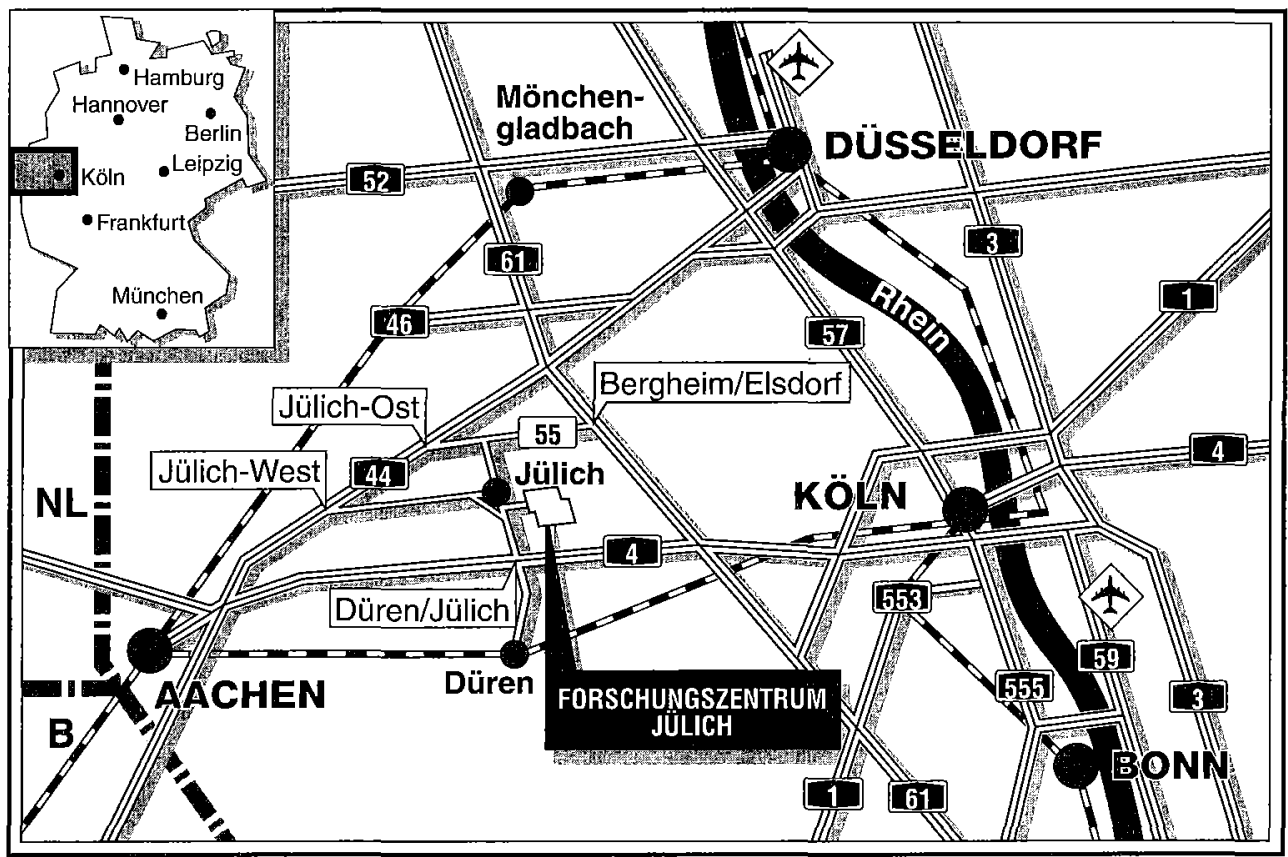

Berichte des Forschungszentrums Jülich ; 3283

ISSN 0944-2952

Institut für Kernphysik Jül-3283

$\mathrm{Zu}$ beziehen durch: Forschungszentrum Jülich $\mathrm{GmbH}$ - Zentralbibliothek D-52425 Jülich · Bundesrepublik Deutschland

Telefon: 02461/61-61 02 - Telefax: 02461/61-61 03 - Telex: 833556-70 kfa d 


\title{
Mesonic Contributions to the Spin and Flavor Structure of the Nucleon
}

\author{
J. Speth* A.W. Thomas ${ }^{* \star}$
}

* Institut für Kernphysik, Forschungszentrum Jülich GmbH, D-52425 Jülich, Germany, and Institut für Theoretische Kernphysik, Universität Bonn

** Department of Physics and Mathematical Physics, and institute for Theoretical Physics, The University of Adelaide, Adelaide S.A. 5005, Australia 


\section{Table of Contents}

1. Introduction 1

2. Elementary Ideas of Deep Inelastic Scattering 3

2.1 Scaling Violations $\quad 7$

2.2. Features of Nucleon Data 9

3. Sullivan Processes 11

3.1. The Convolution Model 12

3.2 Calculation of the Probability Amplitudes $\phi_{B M}$ in TOPT 16

3.3. Meson-Baryon Form Factors 19

$\begin{array}{ll}\text { 3.4. Spin-Averaged Splitting Functions } & 21\end{array}$

3.5. TOPT versus Covariant Perturbation Theory 22

3.6. Polarized Splitting Functions 24

4. Meson Cloud and the Non-Perturbative Sea 27

4.1. Meson-Baryon Form Factors derived from Semi-Inclusive 29 pp-Reactions

4.2. Sea Quark Distributions of the Nucleon 31

4.3. Gottfried Sum Rule and the $\bar{u}-\bar{d}$ Asymmetry 32

4.4. Drell-Yan Processes and $\bar{u}-\bar{d}$ Asymmetry 36

4.5. Polarized Semi-Inclusive Deep-Inelastic Scattering 42

4.6. Exclusive Electroproduction of Pions 43

4.7. Meson Cloud Effects on the Spin-dependent Properties 46 of the Nucleon

5. Mesons in the Proton as Target for Deep-Inelastic Scattering 54

6. Conclusion 58

7. Acknowledgments 59

Appendix A: Lagrangians 60

Appendix B: Vertex Functions $\quad 60$

$\begin{array}{ll}\text { References } & 64\end{array}$

$\begin{array}{ll}\text { Tables } & 69\end{array}$

$\begin{array}{ll}\text { Figure-Captions } & 72\end{array}$

$\begin{array}{ll}\text { Figures } & 75\end{array}$ 


\section{INTRODUCTION}

Since its initial investigation at SLAC in the late 60's, deep inelastic lepton scattering has provided a wonderful tool to explore perturbative QCD and to test many features of the Standard Model. The crucial theoretical tools needed to understand deep-inelastic scattering, namely the operator product expansion and the renormalization group (which are now used throughout particle physics), were developed in parallel with the data taking. Using these techniques one can often eliminate the need to understand the detailed structure of a target in order to make a rigorous test of QCD. Famous examples include the Adler, Gross Llewellyn Smith and Bjorken sum-rules.

With perturbative QCD now well tested and established there are three important frontiers in deep-inelastic scattering. Two of these, namely the behaviour at very small values of Bjorken $x$ (defined in Sect. 2 below) which is currently being explored at HERA and the higher-twist corrections (again see Sect. 2), have traditionally been the domain of high energy physicists. The third frontier, which lies at the boundary of nuclear and particle physics, is our major concern. This is the wealth of information that deep-inelastic scattering data contains on the nonperturbative structure of hadrons (Tho 84a).

The idea of using deep-inelastic scattering to probe non-perturbative structure first reached prominence in connection with the discovery of the nuclear EMC effect $(A u b+83)$. The fact that the structure function of a nucleus was not simply $A$ times the structure function of a free nucleon, even in the valence region, suggested that the quark structure of the nucleon itself may be changed inside nuclear matter. Then quark degrees of freedom would play a vital role in understanding nuclear structure. This is one issue which still needs further study and we refer the interested reader to some recent reviews (GST 95, Arn 94).

We shall focus on the non-perturbative structure of the free nucleon and particular its meson cloud. Although the role of the pion cloud of the nucleon in deep inelastic scattering began with Drell, Yan and Levy in 1970 (DLY 70) and Sullivan (Sul 72 ) in 1972, modern interest in it also sprang from the nuclear EMC effect. Realizing that virtual pion exchange plays a crucial role in nuclear binding, Llewellyn Smith (Lle 83) and Ericson and Thomas (ET 84) explored the consequences for 
nuclear structure functions of an enhancement of the number of virtual pions per nucleon in a nucleus - see also Berger et al. (BCW 84). This led naturally to a renewed interest in the role of the pion cloud in the free nucleon. In particular, it was shown by one of us (Tho 83) that one could put an upper limit on the hardness of the pion-nucleon form factor from the excess of non-strange over strange sea quarks seen in deep-inelastic scattering. It was also realized that the pion cloud necessarily led to an excess of $\bar{d}$ over $\bar{u}$ quarks in the proton.

Since 1983, and particularly since the New Muon Collaboration's confirmation of the excess of $\bar{d}$ over $\bar{u}$ in the proton (Ama+ 91 ), there has been a great deal of theoretical and experimental activity. Much has already been learnt about nucleon structure and an exciting program of experiments needs to be carried out in the next few years to teach us more. Our purpose is to review these developments for the community of physicists fascinated by the strong interaction, whether nuclear or particle physicists by background.

We begin with a brief review of the notation and ideas of deep-inelastic scattering and the general features of the data. Then we turn to the role of the longrange, pion cloud of the nucleon required by chiral symmetry as well as by the Heisenberg Uncertainty Principle! Having reviewed the traditional covariant approach we explain the importance of working in the infinite momentum frame. We explain why a pion cloud leads to important predictions such as an excess of antidown over anti-up quarks, and look at the quantitative predictions.

Having explained the theoretical ideas we turn to the various experiments which can be used to test or constrain them. This includes using exclusive $p p$ reactions to constrain meson-nucleon form factors, measurements of the Gottfried sum-rule, Drell-Yan experiments and polarized deep inelastic scattering. We also discuss semi-inclusive deep inelastic experiments. 


\section{ELEMENTARY IDEAS OF DEEP INELASTIC SCATTERING}

There are a number of fine reviews of the ideas of inclusive lepton scattering at high energy and momentum transfer - the deep inelastic regime (Clo 79, LP 82, Mut 87, Sac 83, Ynd 83, AT 80, Alt 82, Cra+ 83). Our intention here is simply to explain the usual notation and summarize the essential ideas without any attempt at a formal derivation, which would take far too long.

We consider the inclusive scattering of a high energy lepton with initial (final) energy $E\left(E^{\prime}\right)$ and scattering angle $\theta$ from a hadronic target of mass $M$ and initial four-momentum $p$. The spacelike four-momentum transferred to the target is denoted $q$. For an unpolarized target the laboratory differential cross-section for electromagnetic scattering is calculated by contracting the lepton tensor $L_{\mu \nu}$ with the hadronic tensor $W^{\mu \nu}$;

$$
W^{\mu v}=\widetilde{g}^{\mu \nu} W_{1}+\frac{\widetilde{p}^{\mu} \widetilde{p}^{v}}{M^{2}} W_{2},
$$

with

$$
\begin{aligned}
& \widetilde{g}^{\mu \nu}=-g^{\mu \nu}+\frac{q^{\mu} q^{\nu}}{q^{2}}, \\
& \widetilde{p}^{\mu}=p^{\mu}-\frac{p \cdot q}{q^{2}} q^{\mu}
\end{aligned}
$$

and the two arbitrary functions $W_{1}$ and $W_{2}$ contain all the information we can learn about the target from such experiments.

After contracting the tensors and integrating over phase space we find:

$$
\frac{d^{2} \sigma}{d E^{\prime} d \Omega}=\frac{4 \alpha^{2}\left(E^{\prime}\right)^{2}}{q^{4}}\left[\cos ^{2} \frac{\theta}{2} \frac{F_{2}}{v}+2 \sin ^{2} \frac{\theta}{2} \frac{F_{1}}{M}\right]
$$


All of the information concerning the structure of the target is now contained in the structure functions $F_{1}\left(=M W_{1}\right)$ and $F_{2}\left(=v W_{2}\right)$, with $v=E-E^{\prime}$ the photon energy in the laboratory frame) which can depend on at most two variables. One usually chooses those to be the Lorentz invariant quantities $Q^{2}\left(=-q^{2}>0\right)$ and Bjorken $x\left(=-q^{2} / 2 p \cdot q=Q^{2} / 2 M v\right)$. The deep-inelastic regime corresponds to the case where $Q^{2}$ and $v$ are both very large $\left(Q^{2}>2 G e V^{2}, v>1 \mathrm{GeV}\right)$ but $x$ lies in the range $(0,1)$. In the case of neutrino scattering from an unpolarized target we find a third structure function, $F_{3}$, associated with parity violation:

$\frac{d^{2} \sigma^{\nu(\bar{v})}}{d E^{\prime} d \Omega}=\frac{G_{F}^{2}\left(E^{\prime}\right)^{2}}{2 \pi^{2}}\left(\frac{M_{W}^{2}}{M_{W}^{2}+Q^{2}}\right)^{2}\left[\cos ^{2} \frac{\theta}{2} \frac{F_{2}^{\nu(\bar{v})}}{\nu}+2 \sin ^{2} \frac{\theta}{2} \frac{F_{1}^{\nu(\bar{v})}}{M} \pm \frac{E+E^{\prime}}{M \nu} \sin ^{2} \frac{\theta}{2} F_{3}^{\nu(\bar{v})}\right]$.

There has recently been tremendous interest in polarized deep inelastic scattering. In the case where a polarized electron (or muon) scatters from a polarized, spin-1/2 target there are two more structure functions which can be measured, $g_{1}$ and $g_{2}$. Denoting beam and target helicity with arrows top and bottom respectively we find:

$$
\frac{d^{2} \sigma}{d E^{\prime} d \Omega}(\underset{\rightarrow}{\rightarrow} \underset{\leftarrow}{\rightarrow})=\frac{4 \alpha^{2} E^{\prime}}{M \vee Q^{2} E}\left[\left(E+E^{\prime} \cos \theta\right) g_{1}-2 M x g_{2}\right]
$$

Clearly the second term on the right of equ. (2.6) will be negligible (in the deep inelastic region) if $g_{1}$ and $g_{2}$ are of the same order. To determine $g_{2}$ one needs to work with a longitudinally polarized beam and a transversely polarized target. However, even then the cross-section is of order $(1 / Q)$ times that given in equ (2.6):

$$
\frac{d^{2} \sigma}{d E^{\prime} d \Omega}(\vec{\uparrow}-\vec{\downarrow})=\frac{4 \alpha^{2}\left(E^{\prime}\right)^{2}}{M \nu Q^{2} E}\left[g_{1}+\frac{2 E g_{2}}{E+E^{\prime}}\right] \sin \theta
$$


(Here an arrow up indicates that the target is polarized in the direction at right angles to the incident beam, in the scattering plane but pointing opposite to the side where the final lepton exits.)

In the late 1960s tremendous excitement was generated by the discovery at SLAC that the structure functions were almost independent of $Q^{2}$ over a very wide range. That is, they were functions of the single variable - Bjorken $x$. It is very easy to see that this is what one would expect if the nucleon contained a collection of elementary constituents (initially called partons by Feynman but later identified with quarks) with low mass, which do not interact strongly during the deep inelastic collision. For simplicity it is usual to consider this problem in a so-called infinite momentum frame - e.g. One where the nucleon has momentum $P>M$ in the $z$ direction so that its 4-momentum is $p=(P ; 0,0, P)$. Under the assumptions of the parton model, only a parton with fraction $x$ of the momentum of the nucleon can absorb the exchanged photon (or W-boson). Deep inelastic structure functions are then determined by the number density of partons in the nucleon with momentum fraction $x$.

It is usual to define distributions $q^{\uparrow \downarrow}(x)$ which give the number density of quarks in the target with helicity parallel or anti-parallel to that of the target. For example, $u(x) x d x$ gives the fraction of momentum of $u$ quarks in the proton with momentum between $x P$ and $(x+d x) P$ in the infinite momentum frame (and with either helicity). By charge symmetry, $u$ also gives the distribution of $d$ quarks in the neutron, but we note in passing that there are reasons to doubt the accuracy of this for the valence $d$-quarks in the proton at the $5 \%$ level (Sat 92, RTL 94).

The structure functions mentioned earlier are directly related to these distribution functions. For an electromagnetic probe one finds:

$$
\begin{gathered}
F_{1}(x)=\frac{1}{4} \sum_{q} e_{q}^{2}\left(q^{\uparrow}(x)+q^{\downarrow}(x)\right) \\
F_{2}(x)=2 x F_{1}(x)
\end{gathered}
$$




$$
g_{1}(x)=\frac{1}{2} \sum_{q} e_{q}^{2}\left(q^{\uparrow}(x)-q^{\downarrow}(x)\right)
$$

with $e_{q}$ the charge, in units of $e$, of the quark of flavor $q$. Equ. (2.9) is the CallanGross relation and relies on the partons having spin 1/2 and no transverse momentum (in the infinite momentum frame). In general we have

$$
F_{2}=2 x F_{1} \frac{1+R}{1+2 M x / v}
$$

where $R$ is the ratio of cross-sections for absorbing a longitudinal to that for a transverse photon. Experimentally $R$ is small (Das+ 88) $(\leq 0.1)$ for all $x$, for $Q^{2} \geq 5 \mathrm{GeV}^{2}$.

For neutrino scattering from an isoscalar target one finds

$$
F_{2}^{(\bar{v})}=x(u+\bar{u}+d+\bar{d}+s+\bar{s}),
$$

which measures the total quark content of the proton. Even more important, by combining $v$ and $\bar{v}$ data one can measure the combination

$$
F_{3}^{(v, \bar{v})}=(u-\bar{u}+d-\bar{d})
$$

which isolates the excess of quarks over antiquarks - i.e. the valence quark distribution of the nucleon. Clearly we would expect the sum rule (due to Gross and Llewellyn Smith)

$$
\int_{0}^{1} d x F_{3}^{(v, \vec{v})}(x)=3
$$

to be obeyed. It will also be useful to define the n'th moment of a structure function like $x F_{3}, F_{2}$ or $x F_{1}$ as, e.g. 


$$
M_{3 n}=\int_{0}^{1} d x x^{n-2}\left[x F_{3}(x)\right]
$$

\subsection{Scaling violations}

It is clear from the analysis of the experimental data that even in the Bjorken region the structure functions have a weak $Q^{2}$-dependence, and therefore so do the distribution functions which we write as $q\left(x, Q^{2}\right)$. If one sticks to any one data set in order to (partially) avoid systematic errors, this variation of the structure functions (scaling violation) is essentially logarithmic. In order to understand it one must go beyond the naive parton model to QCD.

Suppose we assume that the wavefunction of the target has no highmomentum components (i.e. $p_{T}^{2}<<Q^{2}$ ). Then any $Q^{2}$-dependence can only come from the lepton-quark scattering process. Scaling results if the quark is treated as point-like and the trivial $Q^{2}$-dependence of the Mott cross-section is factored out. On the other hand, in an interaction field theory, the lepton-quark scattering amplitude will involve radiative corrections, some of which add coherently (e.g. wave function and vertex renormalisation) while others are incoherent (e.g. bremsstrahlung). It is well known that such radiative processes lead to corrections which vary logarithmically with the appropriate cut-off scale - in this case $Q^{2}$. This is formally described using the operator product expansion and the renormalization group.

On the basis of the operator product expansion and the renormalization group one can show that the moments of the structure functions can be written as the product of a coefficient function $C_{n}$ (which is independent of the target) and the matrix element of a local operator. In fact one finds that the $Q^{2}$ variation of the moments of the structure functions resides entirely in the coefficient functions which can be calculated by perturbative QCD.

To leading order one finds:

$$
M_{n}\left(Q^{2}\right)=M_{n}\left(Q_{0}^{2}\right)\left(\frac{\alpha\left(Q^{2}\right)}{\alpha\left(Q_{0}^{2}\right)}\right)^{d_{0}^{n}},
$$


and hence for fixed $Q_{0}^{2}$ it is easily shown that

$$
\ln M_{n}\left(Q^{2}\right)=\frac{d_{0}^{n}}{d_{0}^{m}} \ln M_{m}\left(Q^{2}\right)+\text { constant }
$$

so that a log-log plot of any two moments should be a straight line whose slope is predicted by QCD. (The power $d_{0}^{n}$ is known as the anomalous dimension.)

All of the above discussion of $Q^{2}$ evolution applies only to the non-singlet structure functions like $F_{3}$ which cannot involve gluons. The $Q^{2}$ evolution of singlet structure functions such as $F_{2}$ is more complicated because the quarks and gluon operators mix under QCD renormalization. While the corresponding analysis is not much more difficult (it involves a $2 \times 2$ matrix), it would divert us too much to explain it here. Instead we refer to the previously mentioned texts - e.g., there is a summary in Table 2 of the review by Altarelli (Alt 82).

Given an analytic continuation of a set of moments, $M_{n}\left(Q^{2}\right)$, there is a standard method for reconstructing the corresponding function - this is the inverse Mellin transform (IMT):

$$
x F_{3}\left(x, Q^{2}\right)=\frac{1}{2 \pi i} \int_{C-i \infty}^{C+i \infty} d n x^{1-n} M_{3 n}\left(Q^{2}\right),
$$

where $C$ is chosen so that the integral exists. If, as we explained above, the moments of the structure function can be written as a product of a coefficient function, $C_{n}\left(Q^{2}, \mu^{2}\right)$ with the target matrix element, $\left\langle N\left|O_{n}\left(\mu^{2}\right)\right| N\right\rangle$;

$$
M_{2 n}\left(Q^{2}\right)=C_{n}\left(Q^{2}, \mu^{2}\right)\left\langle N\left|O_{n}\left(\mu^{2}\right)\right| N\right\rangle
$$

the inverse Mellin transform, $x F_{3}$, is just a convolution of the IMT of $C_{n}$ (denoted by $C_{3}$ ) and $\left\langle N\left|O_{n}\right| N\right\rangle$ (denoted $F_{3}$ ), viz: 


$$
x F_{3}\left(x, Q^{2}\right)=\int_{x}^{1} \frac{d y}{y} C_{3}\left(x / y, Q^{\dot{2}}, \mu^{2}\right)\left(y F_{3}\left(y, \mu^{2}\right)\right) .
$$

This is an extremely important result. In particular, $C_{3}$ is totally independent of the structure of the target - a property known as factorization. Clearly if we can evaluate the structure function of the target at any renormalization scale $\mu^{2}$, equ. (2.20) allows us to calculate it at all higher values of $Q^{2}$. Higher order QCD corrections do not alter this result in principle, they just make $C_{3}$ harder to compute. For this reason $\mu^{2}$ cannot be too low.

\subsection{Features of Nucleon Data}

Current experiments at HERA are pushing back the kinematic boundaries within which the structure functions are known. For example, one can now reach values of $x$ as low as $2-3 \times 10^{-5}$ at $Q^{2} \sim 2 \mathrm{GeV}^{2}$, whereas fixed target experiments can only get to $x$ of order $8 \times 10^{-4}$ at very low $Q^{2}\left(0.3 \mathrm{GeV}^{2}\right)$. Furthermore, the perturbative evolution of the structure functions can be followed to $Q^{2}$ as high as $10^{3}$ $-10^{5} \mathrm{GeV}^{2}$ at large $x$. For a summary of the present status of the HERA experiments we refer to the proceedings of the recent HERA Workshop on proton, photon and Pomeron structure (HER 96) especially the review by Badelek et al. (Bad+96).

Since our main concern is not with the small $x$ behaviour, it is sufficient to view the data on a linear scale. Fig. 2.1 summarizes an enormous amount of experimental work carried out over the last 20 years with muon and neutrino beams and illustrates some very important features.

First, we note that the anti-quark distribution, which is one half of the sea of virtual - pairs that one sees when looking deep into any hadron, is concentrated in the region below $x=0.3$. In fact, $x \bar{q}(x)$ is typically parameterized as $(1-x)^{\gamma}$ (with $\gamma=7-9)$. Note that the sea quarks, $q_{s}$ with $q_{s} \equiv q-\bar{q}$ - are usually defined to have the same shape as $\bar{q}$. On the other hand, there are suggestions that $s(x)$ may not have the same shape as $\bar{s}(x)$ (ST 87, JT 95, HSS 96) and this will be important to test. 
Later we shall explicitly review the evidence from NMC (Ama+ 91) and NA51 (Bal+ 94) that $\bar{d}>\bar{u}$ which leads to a violation of the Gottfried sum-rule. Finally we note that neutrino induced deep-inelastic scattering to di-muon final states can be used to check the relative strange versus non-strange sea. It seems that strangeness is suppressed for $Q^{2} \sim 5-10 \mathrm{GeV}^{2}$, with (Baz+95)

$$
\frac{2 \bar{s}}{\bar{u}+\bar{d}}=0.45 \pm 0.05
$$

As the second feature of Fig. 1 we note that the valence quarks (recall equ. (13)) dominate for $x$ beyond 0.3. This is confirmed by the closeness of $x F_{3}$ and $F_{2}^{w N}$ for $x \geq 0.3$, after allowing for the electromagnetic charges of the quarks $\left(\frac{5}{18}=\frac{1}{2}\left(\frac{4}{9}+\frac{1}{9}\right)\right)$. In the early days of deep inelastic scattering this provided vital confirmation that the partons of Feynman were, in fact, quarks.

The same counting rules (Drell-Yan-West) which led one to expect the sea quark distributions to behave like $(1-x)^{7}$ as $x \rightarrow 1$ suggest that the valence distributions should behave as, $q_{v}(x)=x^{\alpha}(1-x)^{\beta}$ with $\alpha=-0.5$ (from Regge theory) and $\beta=3$. Of course, QCD evolution implies that $\alpha$ and $\beta$ will also depend slowly on $Q^{2}$ and for recent parameterizations we refer to Badalek et al. (Bad+96) and Martin, Stirling and Roberts MSR 94). The one feature worth noting about the fits discussed there is that they all assume $d_{v} / u_{v} \sim(1-x)$ and hence vanishes as $x \rightarrow 1$. Recent re-analysis of the binding and Fermi motion corrections in the deuteron suggest that this may not be correct (MT 96) and that, in agreement with perturbative QCD (Bro+ 95), $d / u$ may decrease like $(1-x)$ for $x$ below 0.7 but tend to a constant as $x \rightarrow 1$. This needs further testing but one should keep this uncertainty in mind in situations where the actual $d / u$ ratio is important). 


\section{SULLIVAN PROCESSES}

The virtual meson-cloud of the nucleon plays an important role in the understanding of the nucleon-nucleon interaction (MHE 87) and the pion-cloud in particular has always been considered as crucial in understanding the nucleon's long-range structure. For example it was recognized even in the 1950's that the process $n \rightarrow p \pi^{-}$naturally explains the negative tail of the neutron's charge distribution. Furthermore, from PCAC, and the successes of chiral quark models BR 79 , Tho $84 \mathrm{~b}$, TMT 82, AT 83) we know that the nucleon has a pion cloud.

In 1972, Sullivan (Sul 72) showed that in deep-inelastic lepton scattering from a nucleon, the process in which the virtual photon strikes a pion from the meson cloud, and smashes the pion into debris, scales in the Bjorken limit. The reason for this is that, in contrast to processes such as exclusive pion-production, which are suppressed by form factors of the order $1 / Q^{2}$, here the inelastic structure function of the pion itself is probed. The pion contribution to the structure function of the nucleon was investigated later (Tho 83) in connection with SU(3)-symmetry breaking in the quark sea content of the proton. Here it was also pointed out that the pion cloud could be responsible for generating an asymmetry between the $\bar{u}$ and $\bar{d}$ quark content, through the preferred proton dissociation into a neutron and $\pi^{+}$. Furthermore, DIS data on the momentum fraction carried by antiquarks were used to obtain an upper limit on this non-perturbative pionic component and therefore on the number of virtual pions in the nucleon (Kum 91, FMS 89).

These early analyses came to the conclusion that the pionic component is small, which indicates that the pion-nucleon form factor has to be rather 'soft'. This finding was in strong contradiction with the 'hard' form factors which were used in meson-exchange potentials (MHE 87) at that time. As we will see in the following there is not much left from this controversy. Hwang et al. (HSB 91) pointed out that at moderate $Q^{2}$ the total quark sea, including strange quarks, could be connected with the meson cloud. Zoller (Zol 92) showed that the meson-baryon form factors which enter in the analysis of deep-inelastic processes are probed under similar kinematical conditions in semi-inclusive high energy proton-proton reactions. Holtmann et al. (HSS 96) extended the corresponding analysis to include not only pseudoscalar but also vector mesons. The form factors deduced from such 
combined analyses turned out to be a little harder than those in the initial investigations of the sea of the nucleons associated with pions only. On the other side it has been shown (Dei+ 91, TH 90, JHS 94) that more sophisticated mesonexchange potentials can also reproduce the experimental nucleon-nucleon phase shifts with pion-nucleon form factors which are somewhat softer than those which were traditionally used in OBE potentials and are, in fact, close to those derived from DIS.

The concept of the pionic cloud may be extended by taking into account first of all the full pseudoscalar nonet and also the vector meson nonet. These mesons are not only crucial in meson-exchange potentials but it has been known for a long time that they give important contributions to low energy nucleon properties, such as the electromagnetic nucleon form factors or magnetic moments.

The one caution we should add, to which we return in sec. 6 , is that it is not established to what extent one can add incoherently the contributions from mesons of increasing mass. In principle there is the possibility of interference between terms where one reaches the same final state, $X$, from both $\gamma^{*} M B \rightarrow X$ and $\gamma^{*} M^{\prime} B^{\prime} \rightarrow X$. Indeed, as we shall see in sec. 3.6 and 4.7 , for spin-dependent structure functions we require some interference between the final states reached from $\gamma^{*} \pi N^{\uparrow}$ and $\gamma^{*} \pi \Delta^{\uparrow}$. We regard the resolution of this question as being essentially empirical, while noting that apart from the $\rho$-meson the heavier mesons play only a minor role.

\subsection{The Convolution Model}

The convolution model relies on the assumption that the nucleon wave function can be written as the following sum of Fock states

$$
|N\rangle=\sqrt{Z}|N\rangle_{\text {bare }}+\sum_{M B} \int d y d^{2} \vec{k}_{\perp} \phi_{B M}\left(y, k_{\perp}^{2}\right)\left|B\left(y, \vec{k}_{\perp}\right) ; M\left(1-y,-\vec{k}_{\perp}\right)\right\rangle
$$

where $\phi_{\mathrm{BM}}\left(y, k_{\perp}^{2}\right)$ is the probability amplitude to find a physical nucleon in a state consisting of a virtual meson $M$ and a virtual baryon $B$ with longitudinal momentum fractions $y$ and $1-y$ and transverse momenta $\vec{k}_{\perp}$ and $-\vec{k}_{1}$, respectively. $Z$ is the 
standard wave function renormalization factor and can be interpreted as the probability of finding the bare nucleon in the physical nucleon. In the following, we are concerned with first order corrections to the bare nucleon only. Preliminary calculations (Zol 92) suggest that higher order corrections are rather small provided that the form-factors used are not too hard. The infinite momentum frame (IMF) is particularly useful in the study of DIS phenomena. In the infinite momentum frame (i.e., $|\vec{p}| \rightarrow \infty$ with $\vec{p}$ the nucleon momentum c.f. sec. 2) the constituents of the nucleon can be assumed to be free during the DIS reaction time (Fey 72).

The basic hypothesis of the convolution approach is that there are no interactions among the particles in a multi-particle Fock state during the interaction with the hard photon in deep inelastic scattering. This enables one to relate the contribution of a certain Fock state, $B M$, to the nucleon structure function $F_{2}$, to the structure functions of either the struck meson $M$ or the struck baryon $B$ (see Fig. $3.1 \mathrm{a}, \mathrm{b})$ using

$$
\begin{aligned}
& \delta_{M} F_{2}^{N}(x)=\int_{x}^{1} d y f_{M B / N}(y) F_{2}^{M}\left(\frac{x}{y}\right), \\
& \delta_{B} F_{2}^{N}(x)=\int_{x}^{1} d y f_{B M / N}(y) F_{2}^{B}\left(\frac{x}{y}\right),
\end{aligned}
$$

or written in terms of quark distributions:

$$
\delta q_{N}(x)=\int_{x}^{1} f_{M B / N}(y) q_{m}\left(\frac{x}{y}\right) \frac{d y}{y}+\int_{x}^{1} f_{B M / N}(y) q_{B}\left(\frac{x}{y}\right) \frac{d y}{y}
$$

The main ingredients in these formulas are the splitting functions $f_{M B / N}(y)$ and $f_{B M / N}(y)$ which are related to the probability amplitudes $\phi_{\mathrm{BM}}$ in the IMF via

$$
f_{B M / N}(y)=\int_{0}^{\infty} d k_{\perp}^{2}\left|\phi_{B M}\left(y, k_{\perp}^{2}\right)\right|^{2}
$$




$$
f_{M B / N}(y)=\int_{0}^{\infty} d k_{\perp}^{2}\left|\phi_{B M}\left(1-y, k_{\perp}^{2}\right)\right|^{2}
$$

Because the description of the nucleon as a sum of $M B$ Fock states is independent of the DIS process, the relation

$$
f_{M B / N}(y)=f_{B M / N}(1-y)
$$

must hold. It simply expresses the fact that if a meson with longitudinal momentum fraction $y$ is struck by the photon, the remaining part of the nucleon is a baryon with the remaining longitudinal momentum fraction, $1-y$. Moreover this relation automatically ensures global charge conservation

$$
\langle n\rangle_{M B}=\langle n\rangle_{B M}
$$

with $\langle n\rangle_{M B}=\int_{0}^{1} f_{M B / N}(y) d y$ and $\langle n\rangle_{B M}=\int_{0}^{1} f_{B M I N}(y) d y$, and momentum conservation

$$
\langle y\rangle_{M B}+\langle y\rangle_{B M}=\langle n\rangle_{M B},
$$

where $\langle y\rangle_{M B}=\int_{0}^{1} d y y f_{M B}(y)$ and $\langle y\rangle_{B M}=\int_{0}^{1} d y y f_{B M}(y)$ are the average momentum fractions carried by meson $M$ and the virtual baryon $B$, respectively. $\langle n\rangle$ and $\langle y\rangle$ are called the first and second moments of the splitting functions.

It should be noted that, because of the probabilistic interpretation of $\left|\phi_{M B}\left(y, k_{\perp}^{2}\right)\right|^{2}$, the wave function renormalization constant $Z$ can be expressed as

$$
Z=\left(1+\sum_{M B}\langle n\rangle_{M B}\right)^{-1}
$$

The quark distribution functions $q_{N}(x)$ of a nucleon within the Fock state expansion are given as: 


$$
q_{N}(x)=q_{N}^{\text {bare }}(x)+\delta q_{N}(x)
$$

where $q_{N}^{\text {bare }}(x)$ is the quark distribution of the "bare" nucleon (see section 4.2).

In the following, we shall explicitly evaluate the splitting functions $f_{M B}$ and $f_{B M}$, and examine the conditions under which equ. (3.7) is fulfilled. The calculation can be performed in two different ways: (a) within the covariant perturbation theory developed by Feynman, Schwinger and Dyson and (b) in time ordered perturbation theory (TOPT) in the infinite momentum frame, as developed by Weinberg and Drell, Levy and Yan (Wei 66, DLY 70). In the first case relativistic covariance is explicitly fulfilled, but particles are not always on their mass shell. This is a severe drawback in DIS because one is forced to make assumptions about (or to model) (MST 94) the off-mass shell behavior of the hadron structure functions and, in addition, the basic relation in equ. (3.7) is not fulfilled without further assumptions. In TOPT manifest relativistic covariance is lost. However Weinberg (Wei 66) and later Drell, Levy and Yan (DLY 70) have shown that by applying TOPT in the infinite-momentum frame (IMF) one can ensure Lorentz invariance with substantial calculational simplifications which make this method especially suited for DIS - as first pointed out by Bjorken. (Bjo 69).

In his pioneering work Sullivan (Sul 72) used covariant perturbation theory and so did everyone else until the early nineties, when new interest started in these processes, in connection with the violation of the Gottfried sum-rule. Sullivan only considered the process shown in Fig. 3.1 a, where the photon hits the pion. In order to conserve momentum and charge, one must also include the contribution shown in Fig. $3.1 \mathrm{~b}$ where the photon hits the recoiling baryon after the meson is emitted. Previous treatments of this process in the covariant framework were made by several authors (HM 90, KL 91, Kum 91, FMS 89, HSB 91, SS 93, MTS 91). The results, however, were only in qualitative agreement with each other. The main reason was that different procedures were used to fulfill the conservation laws. Moreover, the whole approach was questioned because of the unknown off-massshell behaviour of the hadron (MST 94). For all these reasons, in the more recent work (Zol 92, MT 93, HSS 96) the formalism of TOPT was used in the infinite momentum frame, where these drawbacks do not exist. A review of the covariant formulation and its various problems can be found in ref. (TM 93). In the following, 
we will concentrate on the calculation of various splitting functions within TOPT in the IMF.

\subsection{Calculation of the Probability Amplitudes $\phi_{\mathrm{BM}}$ in TOPT}

The main virtues of this formulation are that off-mass-shell ambiguities in the structure functions of virtual particles can be avoided, and that the meson and baryon splitting functions satisfy equ. (3.7) exactly.

In TOPT the analogue of Fig. 3.1 a involves two diagrams, as shown in Fig. 3.2, where the meson moves forward and backward in time, respectively. The advantage of the infinite momentum frame (IMF), where the target nucleon is moving fast in the $z$ direction (with longitudinal momentum $|\vec{p}|=p_{z} \rightarrow \infty$ ), is that only diagrams involving forward moving mesons have to be considered. Diagrams with backward moving particles give no contributions in the limit $p_{z} \rightarrow \infty$.

The essential ingredients in the convolution model are the probability amplitudes $\phi_{B M}\left(y, k_{\perp}^{2}\right)$. In TOPT in the IMF one is able to write down explicitly the intermediate Fock states

$$
\phi_{B M}\left(\vec{p}, \vec{k}, \vec{k}^{\prime}=\vec{p}-\vec{k}\right)=N_{N} \frac{N_{B}}{(2 \pi)^{\frac{3}{2}}} \frac{N_{M}}{(2 \pi)^{\frac{3}{2}}} \frac{V(\vec{p}, \vec{k}, \vec{p}-\vec{k})}{E_{N}-E_{M}-E_{B}} .
$$

This formula gives the probability amplitude of finding a nucleon with momentum $\vec{p}$ in a Fock state where the baryon $B$ has momentum $\vec{k}$ and the meson $M$ 'momentum $\vec{k}^{\prime}=\vec{p}-\vec{k}$. The factors $(2 \pi)^{\frac{3}{2}}$ come in because we are working in momentum representation. The factors $N_{N}\left(N_{B}\right)$ are the usual fermion wave function normalization factors, $N_{N}=\sqrt{m_{N} / E_{N}}, \quad N_{B}=\sqrt{m_{B} / E_{B}} ; N_{M}$ is a bosonic normalization factor, $N_{M}=1 / \sqrt{2 E_{M}}$. The important feature of TOPT is, in contrast to the covariant perturbation theory, that the intermediate particles are on their mass-shell, i.e. their energies are given by 


$$
E_{N}=\sqrt{m_{N}^{2}+\vec{p}^{2}}, E_{B}=\sqrt{m_{B}^{2}+\vec{k}^{2}}, E_{M}=\sqrt{m_{M}^{2}+\vec{k}^{\prime 2}}
$$

Therefore, one can calculate the vertex function $V$ in equ. (3.12) because one can use on-mass shell wave functions. $V$ depends on a particular model, i.e. on the form of the Lagrangian used. In general $V$ can be written as

$$
V\left(\vec{p}, \vec{k}, \vec{k}^{\prime}\right)=\bar{u}_{N}(\vec{p})_{\alpha} \nu^{\alpha \beta \gamma} \chi_{\beta}\left(\vec{k}^{\prime}\right) \psi_{\gamma}(\vec{k})
$$

where summing and averaging over all possible spin-states is implicitly assumed. $\alpha$, $\beta$ and $\gamma$ are bi-spinor and/or vector indices dependent on the representation used for particles of a given type. $\chi$ and $\psi$ are the wave functions (field operators) of the intermediate meson and baryon, respectively.

$\therefore \quad$ It has been shown (DLY 70) for the $\pi N$ case, that in the infinite momentum limit contributions of Fock states with anti-particles vanish and only contributions with (forward moving) particles survive if appropriate form factors are introduced. This statement is also true for the other Fock states with which we deal. In the IMF the momenta of the particles involved can be parametrized in terms of $y$ and $\vec{k}_{\perp}$ :

$$
\vec{k}=y \vec{p}+\vec{k}_{1}, \quad \vec{k}_{\perp} \cdot \vec{p}=0, \quad \vec{k}^{\prime}=(1-y) \vec{p}-\vec{k}_{\perp} .
$$

Thus, in the limit $p=|\vec{p}| \rightarrow \infty$ :

$$
E_{N}=p+\frac{m_{N}^{2}}{2 p}, E_{B}=|y| p+\frac{m_{B}^{2}+k_{\perp}^{2}}{2|y| p}, E_{M}=|1-y| p+\frac{m_{M}^{2}+k_{\perp}^{2}}{2|1-y| p}
$$

and the energy denominator becomes: 


$$
E_{N}-E_{B}-E_{M}= \begin{cases}2 y p+O\left(\frac{1}{p}\right) & \text { for } y<0 \\ 2(1-y) p+O\left(\frac{1}{p}\right) & \text { for } y>1 \\ \frac{1}{2 p}\left(m_{N}^{2}-M_{B M}^{2}\left(y, k_{\perp}^{2}\right)\right)+O\left(\frac{1}{p^{2}}\right) & \text { for } y \in[0,1]\end{cases}
$$

where $M_{B M}^{2}\left(y, k_{\perp}^{2}\right)$ is the invariant mass squared of the intermediate $B M$ Fock state

$$
M_{B M}^{2}\left(y, k_{\perp}^{2}\right)=\frac{m_{B}^{2}+k_{\perp}^{2}}{y}+\frac{m_{M}^{2}+k_{\perp}^{2}}{1-y}
$$

Only states with $y \in[0,1]$ survive in the IMF-limit. In this limit the probability amplitudes can be expressed as

$$
\phi_{B M}\left(y, k_{\perp}^{2}\right)=\frac{\sqrt{m_{N} m_{B}} V_{M M F}\left(y, k_{\perp}^{2}\right)}{2 \pi \sqrt{y(1-y)}\left(m_{N}^{2}-M_{B M}^{2}\left(y, k_{\perp}^{2}\right)\right)},
$$

with $V_{I M F}$ being the vertex-function in the IMF-limit. In the formula above an extra factor $(\pi p)^{-1 / 2}$ has been taken out. It would cancel when going to probability densities by an appropriate factor of the Jacobian of the transformation (3.15).

Formula (3.19) can be used to calculate $\phi_{B M}\left(y, k_{\perp}^{2}\right)$ for all cases of interest see refs. (Zol 92, TM 93, HSS 93, HSS 96). Using TOPT this way has one disadvantage in comparison with the typical calculation involving traces: one has to calculate $V_{I M F}$ for every spin configuration and calculate the spin average afterwards. On the other hand this turns out to be most useful for polarized DIS, as we will see in section 4.5 . 


\subsection{Meson-Baryon Form Factors}

The symmetry given by equ. (3.7) is automatically fulfilled for vertices involving point-like particles. Because of the extended structure of the hadrons involved one has to introduce phenomenological vertex form factors, which parametrize the unknown microscopic effects. It is interesting to mention that Drell et al. (DLY 70) also had to introduce cut-offs in the transverse-momentum $k_{\perp}^{2}$, when they derived the parton model for deep inelastic electron scattering within the same formalism. For these reasons, the vertex function, $V\left(y, k_{\perp}^{2}\right)$, should be replaced by $V^{\prime}\left(y, k_{\perp}^{2}\right)=G\left(y, k_{\perp}^{2}\right) V\left(y, k_{\perp}^{2}\right)$. Equation (3.7) now imposes a severe restriction on these form factors:

$$
G_{B M}\left(y, k_{\perp}^{2}\right)=G_{M B}\left(1-y, k_{\perp}^{2}\right)
$$

The form factors often used in meson exchange models and convolution models are functions of $t$ only, the four-momentum squared of the meson, and do not satisfy equ. (3.20). In terms of the IMF variables $y$ and $k_{\perp}^{2}, t$ is given by (for comparison we also write down $u$, the four-momentum squared of the intermediate baryon)

$$
\begin{aligned}
& t=\frac{k_{\perp}^{2}}{y}-(1-y)\left(\frac{m_{B}^{2}}{y}-m_{N}^{2}\right) \\
& u=\frac{k_{\perp}^{2}}{1-y}-y\left(\frac{m_{M}^{2}}{1-y}-m_{N}^{2}\right)
\end{aligned}
$$

Thus, form factors depending on $t$ only, like the dipole form factor

$$
G(t)=\left(\frac{\Lambda^{2}-m m_{M}^{2}}{\Lambda^{2}-t}\right)^{2}
$$


are ill-defined for the convolution models. They do not conserve basic quantities like charge and momentum. One simple method to obtain form factors with the right symmetry is to multiply a $t$-dependent form factor by a $u$-dependent one with the same functional form, with $m_{M}$ replaced by $m_{B}$

$$
G_{s y m}(t, u)=G\left(t, m_{M}\right) G\left(u, m_{B}\right)
$$

The importance of using such symmetric form factors was noticed only recently (Zol 92, HSS 93). Another possible approach, namely to fix the cut-off parameters to assure number sum rules (global charge conservation) (see (SMM 92, DT 92)), is somewhat arbitrary, and does not guarantee momentum conservation, and gives rise to very different functional behavior of $f_{N \pi}(y)$ and $f_{\pi N}(1-y)$.

In recent publications dipole parametrizations (TM 93)

$$
G_{N B M}\left(y, k_{\perp}^{2}\right)=\left(\frac{\Lambda^{2}+m_{N}^{2}}{\Lambda^{2}+M_{B M}^{2}\left(y, k_{\perp}^{2}\right)}\right)^{2}
$$

and exponential forms (Zol 92, HSS 93, HSS 96) have been used

$$
G_{N B M}\left(y, k_{\perp}^{2}\right)=\exp \left(\frac{m_{N}^{2}-M_{B M}^{2}\left(y, k_{\perp}^{2}\right)}{2 \Lambda^{2}}\right)
$$

with the invariant mass, $M_{B M}^{2}$, defined in equ. (3.18). Using equs. $(3.21,3.22)$ one can show, that the exponential form given in equ. (3.24b) is equivalent to the symmetrized form

$$
G_{N B M}(t, u)=\exp \left(\frac{t-m_{M}^{2}}{2 \Lambda^{2}}\right) \exp \left(\frac{u-m_{B}^{2}}{2 \Lambda^{2}}\right)
$$




\subsection{Spin-Averaged Splitting Functions}

Equations (3.5) and (3.19) allow one to write the spin-averaged splitting functions as:

$$
f_{B M / N}\left(y, k_{\perp}^{2}\right)=\frac{1}{4 \pi^{2}} \frac{m_{N} m_{B}}{y(1-y)} \frac{\left|V_{M M F}\right|^{2}}{\left[m_{N}^{2}-M_{B M}^{2}\left(y, k_{\perp}^{2}\right)\right]^{2}}
$$

where the vertex functions define the model. The vertex functions for the Lagrangians listed in Appendix $\mathrm{A}$ are given in Appendix B. For the $N \pi$ and $\Delta \pi$ Fock states we find:

$$
f_{N \pi / N}(y)=\frac{g^{2}}{16 \pi^{2}} \frac{1}{y^{2}(1-y)} \int_{0}^{\infty} d k_{\perp}^{2}\left|G_{N \pi}\left(y, k_{\perp}^{2}\right)\right|^{2} \frac{m_{N}^{2}(1-y)^{2}+k_{\perp}^{2}}{\left[m_{N}^{2}-M_{N \pi}^{2}\left(y, k_{\perp}^{2}\right)\right]^{2}}
$$

$f_{\Delta \pi / N}(y)=\frac{f^{2}}{96 \pi^{2} m_{\Delta}^{2}} \frac{1}{y^{4}(1-y)} \int_{0}^{\infty} d k_{\perp}^{2}\left|G_{\Delta \pi}\left(y, k_{\perp}^{2}\right)\right|^{2} \frac{\left[\left(m_{\Delta}+y m_{N}\right)^{2}+k_{\perp}^{2}\right]^{2}\left[\left(m_{\Delta}-y m_{N}\right)^{2}+k_{\perp}^{2}\right]}{\left[m_{N}^{2}-M_{\Delta \pi}^{2}\left(y, k_{\perp}^{2}\right)\right]^{2}}$

and finally for the vector mesons:

$$
\begin{aligned}
& f_{N V / N}(y)=\frac{3}{16 \pi^{2}} \int_{0}^{\infty} d k_{\perp}^{2} \frac{\left|G_{N V}^{2}\left(y, k_{\perp}^{2}\right)\right|}{\left[m_{N}^{2}-M_{N V}^{2}\left(y, k_{\perp}^{2}\right)\right]^{2}}\{ \\
& g_{V N N}^{2}\left[\frac{\left[k_{\perp}^{2}+(1-y)^{2} m_{N}^{2}-y m_{V}^{2}\right]^{2}+2 m_{V}^{2}\left[\left(1+y^{2}\right) k_{\perp}^{2}+(1-y)^{4} m_{N}^{2}\right]}{y^{2}(1-y)^{3} m_{V}^{2}}\right] \\
& +f_{V N N}^{2} \frac{1}{y^{4}(1-y)^{3} m_{V}^{2}}\left[\left[k_{\perp}^{2}+(1-y)^{2} m_{N}^{2}-y m_{V}^{2}\right]^{2}\left[(1-y)^{4} m_{N}^{2}+(1+y)^{2} k_{\perp}^{2}\right]\right. \\
& \left.+16_{y}^{2} m_{\nu}^{2}\left[k_{\perp}^{4}+3 m_{N}^{2}(1-y)^{2} k_{\perp}^{2}+2(1-y)^{4} m_{N}^{4}\right]\right] \\
& \left.+g_{V N N} f_{V N N} m_{N}\left[\frac{\left[k_{\perp}^{2}+(1-y)^{2} m_{N}^{2}-y m_{V}^{2}\right]^{2}-8 y m_{V}^{2}\left[k_{\perp}^{2}+(1-y)^{2} m_{N}^{2}\right]}{y^{3}(1-y) m_{V}^{2}}\right]\right\}
\end{aligned}
$$


As examples of the various splitting functions we show $f_{N \pi}, f_{\Delta \pi}$ and $f_{N p}$ in Fig. 3.3 ( Hol 95). TOPT and a cut-off deduced from high-energy scattering data have been used with parameters given in section $4: 1$. The $\rho N$-splitting function peaks at larger $y$ compared to the $\pi N$ because the heavier $\rho$-meson carries more momentum. On the other hand the $\pi \Delta$-splitting function peaks at somewhat lower $y$ compared with the $\pi N$ case - because the $\Delta$ is slightly heavier than the nucleon.

The results obtained by means of TOPT in the IMF are exactly the same as those derived with covariant perturbation theory. However, in TOPT it is natural to use form factors which depend on $k_{\perp}^{2}$ and therefore implicitly on $t$ and $u$, whereas in covariant perturbation theory the natural choice are form factors which depend on $t$ only. This is incorrect, as mentioned before. After transforming variables $\left(y, k_{\perp}^{2}\right) \rightarrow(u, t)$ (equs. 3.21, 3.22) one obtains the standard results for $N \pi$ (Sul 72, Kum 91, DT 92, MTS 91) and $\Delta \pi$ (Kum 91, MTS 91) contributions. This should be the case, because TOPT is just a different way to calculate diagrams. There is, however, one point which requires a separate discussion in the next section.

\subsection{TOPT versus Covariant Perturbation Theory}

If the vertex functions used contain a derivative of the meson field, care must be taken or one will find differences between TOPT and covariant perturbation theory. To illustrate this point let us consider the example of the pseudovector $N \pi N$ vertex, given by

$$
L_{p \nu}=\bar{u} \gamma_{5} \gamma^{\mu} \partial_{\mu} \pi u
$$

where for simplicity the coupling constant and isospin structure have been suppressed. The standard covariant techniques lead to the splitting function of the meson 


$$
\begin{gathered}
f_{M B / N}^{p \nu}(y)=\frac{1}{16 \pi^{2}} \frac{1}{(1-y)^{2} y} \int_{0}^{\infty} d k_{\perp}^{2} \mid G\left(1-y, k_{\perp}^{2}\right)^{2} \\
\frac{\left(m_{B}+m_{N}\right)^{2}\left[\left(m_{N}(1-y)-m_{B}\right)^{2}+k_{\perp}^{2}\right]}{\left[m_{N}^{2}-M_{B M}^{2}\left(1-y, k_{\perp}^{2}\right)\right]^{2}} .
\end{gathered}
$$

The result for the baryon is:

$$
\begin{aligned}
f_{B M / N}^{p v}(y) & =\frac{1}{16 \pi^{2}} \frac{1}{(1-y)^{2} y} \int_{0}^{\infty} d k_{\perp}^{2}\left|G\left(y, k_{\perp}^{2}\right)\right|^{2} \\
\therefore & \left(m_{N}^{2} m_{B}^{2}(1-y)^{2}+k_{\perp}^{2}\left(m_{B}+m_{N}\right)^{2}-2 m_{N} m_{B}\left(y m_{M}^{2}+k_{\perp}^{2}\right)+\frac{1}{(1-y)^{2}}\left(y m_{M}+k_{\perp}^{2}\right)^{2}\right) \\
& \quad \frac{1}{\left[m_{N}^{2}-M_{B M}^{2}\left(y, k_{\perp}^{2}\right)\right]^{2}} .
\end{aligned}
$$

Here as mentioned before the two results are not related by equ. (3.7), which leads to a violation of charge- and momentum conservation. The reason for this puzzle is that by using a derivative coupling, an additional off-shell dependence is introduced into the vertex function, which cannot be suppressed in the IMF-limit. A way out is to use TOPT (Zol 92, HSS 93). Here, however, the problem arises how to choose the meson energy in the vertex. In principle, there are two possible prescriptions:

A) One uses in the vertex the meson four-momentum $q^{\mu}: \bar{u}_{N} \gamma_{s} \gamma_{\mu}(-i) k^{\prime \mu} u_{B}$ i.e. the meson energy in the vertex is $E_{M}$ (equ. 3.16). With this form of the vertex one reproduces the baryon splitting function given by equ. (3.30). The meson splitting function is related to this result by equ. (3.7).

B) Instead of $k^{\prime \mu}$ one uses the difference of the baryon four-momenta $p^{\mu}-k^{\mu}: \bar{u}_{N}(p) \gamma_{s} \gamma_{\mu}(-i)(p-k)^{\mu} u_{B}(k)$, i.e. the meson energy in the vertex is $E_{N}-E_{B}$. With this prescription one gets the meson splitting function given by equ. (3.29). Again the corresponding result for the baryon splitting function fulfills equ. (3.7). 
Thus TOPT, in contrast to a covariant calculation, is consistent with the convolution approach. The remaining point to clarify is which one of the two prescriptions one should use. The natural choice is prescription $B$, because in this prescription the splitting functions for the pseudovector case are identical to those of the pseudoscalar case, if the coupling constants are identified properly. Moreover, in this prescription the structure of the vertex is due to the baryonic current only.

In Fig. 3.4 a comparison is made between the splitting functions $f_{\pi N}(y)$, calculated in covariant and time-ordered perturbation theory, respectively. While the dependence on the functional form of the formfactor within TOPT is weak, the results obtained with the two different formalisms deviate appreciably. Even more severe is the violation of the basic symmetry, $f_{n N}(y)=f_{N \pi}(1-y)$, in the covariant formalism. Whereas it is automatically fulfilled in TOPT, the two functions, shown in Fig. 3.5, do not have this symmetry at all - the most obvious difference being that $f_{N \pi}(1-y)$ is finite at $y=1$.

One should bear this result in mind if one calculates semi-exclusive reactions like deep-inelastic electroproduction of neutrons, from which one aims to measure the pion structure function at small $x$. As 3-momenta are not conserved in the splitting function, if calculated in the covariant formalism, the neutron- and pionmomentum are not connected with each other, as discussed above.

\subsection{Polarized Splitting Functions}

The same approach is convenient for the extension to deep inelastic scattering of polarized particles (Zol 93, HSS 96). Let us introduce the probability amplitudes $\phi_{B M}^{\lambda \lambda^{\prime}}\left(y, k_{\perp}^{2}\right)$, which describe a nucleon with positive helicity $(+1 / 2)$, being in a Fock state $B M$ with baryon helicity $\lambda$ and meson helicity $\lambda^{\prime}$. To calculate $\phi_{B M}^{2 \lambda}\left(y, k_{\perp}^{2}\right)$, equ. (3.19) can be used with the spin-averaged vertex function $V_{I M F}$ replaced by an appropriate helicity dependent one $V_{M M F}^{\lambda \lambda}$ (see Appendix B). By analogy with the spin-averaged splitting function (see equs. (3.5) and (3.6) we introduce the polarized splitting functions $f_{B M / N}^{\lambda}(y)$ and $f_{M B / N}^{\lambda^{\prime}}(y)$, which we define as 


$$
\begin{gathered}
f_{B M I N}^{\lambda}(y)=\sum_{\lambda^{\prime}} \int_{0}^{\infty} d k_{\perp}^{2}\left|\phi_{B M}^{\lambda \lambda^{\prime}}\left(y, k_{\perp}^{2}\right)\right|^{2}, \\
f_{M B / N}^{\lambda^{\prime}}(y)=\sum_{\lambda^{\prime}} \int_{0}^{\infty} d k_{\perp}^{2}\left|\phi_{B M}^{\lambda \lambda^{\prime}}\left(1-y, k_{\perp}^{2}\right)\right|^{2} .
\end{gathered}
$$

For simplicity, let us specialize our discussion to the $N \pi$ case and use the more suggestive notation $f^{\uparrow}(y)=f_{N \pi / N}^{+1 / 2}(y)$ and $f^{\downarrow}(y)=f_{N \pi / N}^{-1 / 2}(y)$. Applying the idea of the convolution model to the polarized quark distributions $q^{\uparrow}(x), q^{\downarrow}(x)$ (the quark distribution of quarks with flavour $q$ and spin parallel or antiparallel to the nucleon spin) leads to the following relations:

$$
\begin{aligned}
& \delta q_{N}^{\uparrow}(x)=\int_{x}^{1} f^{\uparrow}(y) q_{B}^{\uparrow}\left(\frac{x}{y}\right) \frac{d y}{y}+\int_{x}^{1} f^{\downarrow}(y) q_{B}^{\downarrow}\left(\frac{x}{y}\right) \frac{d y}{y}, \\
& \delta q_{N}^{\downarrow}(x)=\int_{x}^{1} f^{\uparrow}(y) q_{B}^{\downarrow}\left(\frac{x}{y}\right) \frac{d y}{y}+\int_{x}^{1} f^{\downarrow}(y) q_{B}^{\uparrow}\left(\frac{x}{y}\right) \frac{d y}{y} .
\end{aligned}
$$

Taking the sum and the difference gives:

$$
\begin{gathered}
\delta q_{N}(x)=\int_{x}^{1} f(y) q_{B}\left(\frac{x}{y}\right) \frac{d y}{y}, \\
\delta \Delta q_{N}(x)=\int_{x}^{1} \Delta f(y) \Delta q_{B}\left(\frac{x}{y}\right) \frac{d y}{y},
\end{gathered}
$$

with $\Delta q(x)=q^{\uparrow}(x)-q^{\downarrow}(x)$. Here $f(y)$, the splitting function for the unpolarized (spin averaged) case, is given by the sum of $f^{\uparrow}(y)$ and $f^{\downarrow}(y) ; \Delta f(y)$, the splitting function for the polarized case, by the difference. It is important to note, that the splitting functions $f^{\uparrow}(y)$ and $f^{\downarrow}(y)$ are independent of each other (Zol 93), i.e. there are no simple relations, like Clebsch-Gordan coefficients between them. 
As an example, we show in Figure 3.6 the corresponding splitting functions for the $N \pi$ Fock component. As seen from the figure the non-spin-flip (dashed line) and the spin-flip (dotted line) contributions are different. The spin-flip contribution dominates at large momentum fraction of the nucleon (Zol 93). The spin-flip contribution is totally due to the presence of perpendicular momentum in the pionnucleon wave function. This explains the sensitivity of the spin-flip splitting function to the cut-off parameter of the vertex form factor. It also leads to a similar sensitivity of the polarized splitting function $\Delta f=f^{\uparrow}-f^{\downarrow}$ (dash-dotted line). Here, if integrated over $y$, the spin-flip and non-spin-flip contributions almost cancel each other. There is no deep reason for this cancellation; it is rather the consequence of the cut-off parameter choice. In the present calculation, the cut-off parameter $(\Lambda=1.08 \mathrm{GeV})$ has been adjusted to the neutron production data [see section 4.1]. This cancellation has consequences for the spin content of the nucleon as we will discuss in section 4.7 .

Similar formulas can be derived analogously for other Fock states. For particles with higher spin additional assumptions have to be made. For example, in the $\Delta \pi$ case one has to distinguish between the $q^{\uparrow}(x)$ distributions in a $\Delta$ with helicity $1 / 2$ and the similar distribution (we call it $q^{n}(x)$ ) in a $\Delta$ with helicity $3 / 2$. Assuming the relation $q^{\Uparrow}(x)=3 q^{\uparrow}(x)$ for the bare $\Delta$, which can be easily checked using SU(6) symmetry, it is possible to obtain an equation similar to equ. (3.26).

In the most general case, assuming that the polarized quark distribution of different helicity states are the same (except helicity factors) one gets:

$$
\begin{aligned}
\delta \Delta q_{N}(x) & =\int_{x}^{1} \Delta f_{B M / N}(y) \Delta q_{B}\left(\frac{x}{y}\right) \frac{d y}{y}+\int_{x}^{1} \Delta f_{M B / N}(y) \Delta q_{M}\left(\frac{x}{y}\right) \frac{d y}{y} \\
& +\int_{x}^{1} \Delta f_{\left(B^{\prime} B\right) M / N}(y) \Delta q_{B B^{\prime}}\left(\frac{x}{y}\right) \frac{d y}{y}+\int_{x}^{1} \Delta f_{B\left(M M^{\prime}\right)}(y) \Delta q_{M M^{\prime}}\left(\frac{x}{y}\right) \frac{d y}{y},
\end{aligned}
$$

with

$$
\Delta f_{X Y / N}(y)=\sum_{\lambda} 2 \lambda f_{X Y / N}^{\lambda}(y)
$$


where the sum runs over all possible helicities of particle $X$.

From our experience with the calculation of axial coupling constants (c.f. sec. 4.7), it is vital to include terms in which (e.g.) the polarized-photon- $N$ interaction leads to the same final states as the polarized-photon- $\Delta$ interaction (ST 88, STL 90, Zol 93, HSS 96). Indeed, this is precisely the reason that perturbation theory works so well when one carries through the renormalization program in the cloudy bag model (Tho 84b, TMT 82) These cross terms for baryons and mesons are, respectively, the third and fourth terms on the rhs of equ. (3.37).

The formalism outlined here has been applied to the semi-leptonic decay and the "missing" spin of the nucleon.

\section{MESON CLOUD AND THE NON-PERTURBATIVE SEA}

There exist numerous attempts to link quark models with DIS. The main idea behind such approaches is that the QCD evolution implies a decreasing number of sea-quarks (i.e. virtual $q \bar{q}$-pairs) as the momentum scale, $Q^{2}$, decreases. Since most quark models involve three valence quarks (sometimes a small $q \bar{q}$-admixture is also considered), one would expect the model to relate to DIS at some low momentum scale, $Q^{2}=Q_{0}^{2}($ LeY $+75, \mathrm{PP} 76)$. There has been considerable success in reproducing the experimental data (STL 90, SST 91, ST 94, SHT 95). However, two important points have to be borne in mind: (I) The evolution equation is first or second order in the running coupling constant and one must therefore worry about applying it below $1(\mathrm{GeV} / \mathrm{c})^{2}$. We note, however, that the agreement between calculations using leading order and next to leading order evolution is very close. (II) In addition, many of the early calculations did not respect chiral symmetry. That is, no quark model can be consistent with the known symmetries of QCD unless pions are included. Since, as we have seen, pions contain a valence $q \bar{q}$ pair, this pion cloud necessarily constitutes a sea of $q \bar{q}$ pairs in the nucleon.

Thomas (Tho 83), and some years later Frankfurt et al. (FMS 89), used the fact that this pion contribution breaks the SU(3)-flavour symmetry of the sea distribution to put a limit on the hardness of the $\pi N N$ form factor which controls the 
pion emission - see equ. (3.26). In Thomas' case the limit is such that a chiral bag radius cannot be too small, say $\mathrm{R}=0.87 \pm 0.10 \mathrm{fm}$. In ref. (FWS 89) the $\pi N \Delta$ coupling was also taken into account. In that case the bag radius had to be larger than $1 \mathrm{fm}$. Frankfurt et al. also concluded that meson-exchange models for the $N N$-interaction are "not well justified on the microscopic level". In both calculations only the SU(3) breaking part of the proton sea, i.e. $x\left(\frac{\bar{u}(x)+\bar{d}(x)}{2}-\bar{s}(x)\right)$ was attributed to the $\bar{q} q-$ contribution of the pion. Hwang, Speth and Brown (HSB 91) took a different point of view: they suggested that at moderate momentum transfer a significant fraction of the proton $\bar{q} q$ sea could be due to the nonperturbative meson contribution. (They also included $K$ and $K^{*}$ mesons.) In that case, the amount of $\bar{u}, \bar{d}$-antiquarks which are due to mesons can be somewhat larger, pushing the allowed cut-off towards $1 \mathrm{GeV}$. In a recent publication Koepf, Frankfurt and Strikman (KFS 96) came to similar conclusions. (Note that they used covariant perturbation theory which suffers from the problems discussed in chapter 3 ). In the past few years, the $\pi N N$ form factor used in meson-exchange potentials has been better understood (Jan+ 93) and meson-exchange potentials with much softer $\pi N N$ form factors have been constructed (HHT 94, Jan 95). Therefore one may conclude that there is no longer any inconsistency disagreement between meson-exchange models and DIS.

The Jülich group (HSS 96, Hol 95) has placed considerable importance on using, in equs. (3.26 - 3.27a), meson-baryon vertices which are derived from other experimental data - like semi-exclusive neutron production in high-energy protonproton collisions. They calculated the corresponding meson contributions to the proton and neutron sea-quark distributions using the experimentally known pion structure function. Moreover, it has been shown that this process can also be reversed; if the $\pi N N$ vertex is known, one can obtain from semi-inclusive deep inelastic scattering processes, the pion structure function down to very small $x$, which will allow one to extract the sea-quark structure function of the pion ( $\mathrm{Hol}+94)$. In the following, we will discuss this point of view in more detail. 


\subsection{Meson-Baryon Form Factors derived from Semi-Inclusive $p p$-Reactions}

The functional form of the form factors used in deep-inelastic scattering is the same as the form used in meson-exchange $N N$ potentials: (a) monopole- and dipole form as used in the Bonn-potential (MHE 87), (b) Gaussian form as used by the Nijmegen-group (NRS 78). The following analyses by Holtmann et al. (HSS 96) have been performed with a Gaussian form factor, as given in equ. (3.24b), where $\Lambda$ is the inverse of a radius. In some models, like the cloudy bag, the radius is connected with the confining region of the valence quarks (Tho $84 \mathrm{~b}$ ). In other models this connection with the confining region is not so obvious.

In order to fix the cut-off parameters, $\Lambda$, of the form factors, the authors of ref. (HSS 96) used high-energy particle production data. They included in their model the pseudoscalar and vector meson octet. A similar analysis, including the pseudoscalar mesons only, was first performed by Zoller (Zol 92). The neutron and Lambda production data in the reactions $p p \rightarrow n X$ and $p p \rightarrow \Lambda X$ seems to be best tailored for extracting the cut-off parameters for the $N \pi$ and $N \rho$ as well as for the $\Lambda K$ and $\Lambda K^{*}$ Fock space components. If one restricts the analysis to data with relatively low exchanged four-momenta it is reasonable to assume that the neutron and the Lambda are produced by a simple one-boson-exchange mechanism (OBE) as shown in Fig. 4.1. In complete analogy to modern meson-exchange potentials one also assumes non-Reggeized mesons. Therefore, the region $y \rightarrow 0$ and $(1-y) \rightarrow 0$ should not be considered. Fortunately, neither of these regions is important for evaluating the splitting functions. In the OBE approximation the invariant cross section for $p p \rightarrow B X$ production has the form:

$$
\begin{aligned}
& E \frac{d^{3} \sigma(p p \rightarrow B X)}{d^{3} p}=\frac{y}{\pi} \frac{d^{2} \sigma}{d y d k_{\perp}^{2}} \\
& =\left|\phi_{B M}\left(y, k_{\perp}\right)\right|^{2} \cdot \sigma_{t o l}^{M p}(s(1-y)) .
\end{aligned}
$$

The probability amplitudes, $\phi_{B M}$, are defined in equ. (3.19), where $y$ is the longitudinal momentum fraction of the baryon with respect to the momentum of the incoming proton and the corresponding transverse momentum. Moreover $\sigma_{\text {tot }}^{M p}$ is the 
total meson-proton scattering cross-section. For $\pi p$ and $K p$ the total cross sections, $\sigma_{\text {tot }}^{\pi p}$ and $\sigma_{t o t}^{K p}$, are known experimentally; the corresponding vector meson cross sections are assumed to be equal to the pseudo-scalar ones. It is important to bear in mind that the hadronic and photonuclear reactions are kinematically identical.

The results of such an analysis are shown in Fig. 4.2 (for details see Refs. (HSS 96, Hol 95). As a criterion for the fit, it was assumed that the theoretical result must not exceed the experimental data. This is well fulfilled in the case of the neutron and not so well in the case of the $\Lambda$, where the data are not so good. For low $k_{\perp}^{2} \pi$-and $K$-exchange, respectively, are the dominant contributions, whereas the vector mesons do not play a role. For higher $k_{\perp}^{2} \rho$ - and $K^{*}$-exchange become the dominant mechanism. It turns out that one can choose the same cut-off parameter, $\Lambda_{M p}=1.08 \mathrm{GeV}$, for pseudo-scalar and vector mesons. If one considers this parameter as universal for the meson-baryon-octet, then heavier mesons are strongly supressed and do not play a role. A corresponding analysis has also been performed for $\Delta^{++}$production. Here the cut-off parameter has to be chosen somewhat small, $\Lambda_{M \Delta}=0.98 \pm 0.05 \mathrm{GeV}$. However this value is much less reliable than the previous one because the data are not very convincing. For example, the double differential cross sections vary by up to a factor of 2 , depending on the assumed background. The $y$-integrated spectra are more precise, with errors of about $30 \%$. The authors of ref. (HSS 96) also found that their results do not depend sensitively on the analytic form of the form factors (exponential, monopole or dipole) as long as they fulfill the basic symmetry given in equ. (3.7). If one uses conventional form factors which only depend on $t$, a similar analysis to that shown in Fig. 4.2 fails already for $k_{\perp}^{2} \geq 0.3(\mathrm{GeV} / \mathrm{c})^{2}$.

Table 4.1 gives the probabilites [in \%] of finding the various Fock states in the nucleon. Note that the total probability of finding pion- and rho- configurations is nearly $40 \%$. This is in agreement with the results of the Adelaide group (TM 93). In fact it is amazing how close these probabilities are to those found in the cloudy bag model more than a decade ago (Tho $84 \mathrm{~b}$ ). On the other hand, configurations with strange baryons and mesons are very small in the present model. Here, the strange sea of the nucleon is mainly due to the strange sea in the mesons, as we will discuss 
later. Thus the contribution of the strange sea to the spin of the nucleon is negligibly small in this model.

\subsection{Sea-Quark Distributions of the Nucleon}

In order to calculate the quark distribution function within the Fock state expansion, see section 3.1, one needs as input the quark distribution functions of the mesons and the bare baryons. For the quark distribution in the pion, a recent parameterization by Sutton et al. (Sut+ 92) has been used. By applying SU(3) symmetry, the distributions of the other mesons were (approximately) determined. Unfortunately, the sea quark distribution of the pion is practically unknown, and one must therefore make assumptions about its magnitude which simultaneously influence the assumptions about the sea of the bare nucleon. In order to determine the valence distribution of the bare nucleon self-consistently it was parameterized in such a way that the theoretical valence distributions calculated from equ. (3.12) agreed with the phenomenological parameterizations. Two different scenarios have been considered: I (Fit I) no sea in the bare nucleon, instead $40 \%$ of the momentum of the meson is carried by sea-quarks; II (Fit II) $20 \%$ of the meson momentum is carried by the sea, but then one needs a small sea in the bare nucleon. An experimental method to test this has been proposed by Londergan et al. (Lon+ 94). This sea is assumed to be symmetric in $\bar{u}$ and $\bar{d}$, whereas the strange sea is supressed by $50 \%$. Both fits agree within the errors with the data. The results of ref. (Hol 95) are shown in Figs. 4.3 and 4.4. In the latter, the changes, especially in the $u$-valence distribution, due to the coupling to the mesons are clearly seen. One also recognizes the asymmetry in the $\bar{u}$ and $\bar{d}$ distributions which is exclusively due to the Fock space expansion. The model has also been compared with the CCFR-data (Fou+ 90). As shown in Table 4.2 the agreement between theory and experiment is satisfying.

Recently Steffens et al. (SHT 95) used quark model wave functions to calculate the bare valence quark distribution function. As shown in Fig. 4.5 the agreement with the phenomenological distribution is also quite good. 


\subsection{Gottfried Sum Rule and the $\bar{u}-\bar{d}$ Asymmetry}

The Gottfried sum rule is perhaps the most famous consequence of $\mathrm{SU}(2)$ flavour symmetry of the sea. Because it measures the $x$-integrated difference between the proton and neutron structure functions, it is sensitive only to the nonsinglet $\mathrm{SU}(2)$ content of the nucleon. Let us first define the quantity

$$
S_{G}(x, 1)=\int_{x}^{1} \frac{d x^{\prime}}{x^{\prime}}\left(F_{2}^{\mu p}\left(x^{\prime}\right)-F_{2}^{\mu n}\left(x^{\prime}\right)\right)
$$

Relating the proton and neutron structure functions to the quark distributions in the proton (i.e. using charge symmetry), we have

$$
\begin{aligned}
S_{G}(x, 1) & =\frac{1}{3} \int_{x}^{1} d x^{\prime}\left(u\left(x^{\prime}\right)+\bar{u}\left(x^{\prime}\right)-d\left(x^{\prime}\right)-\bar{d}\left(x^{\prime}\right)\right) \\
& =\frac{1}{3} \int_{x}^{1} d x^{\prime}\left(u_{v}\left(x^{\prime}\right)-d_{v}\left(x^{\prime}\right)\right)-\frac{2}{3} \int_{x}^{1} d x^{\prime}\left(\bar{d}\left(x^{\prime}\right)-\bar{u}\left(x^{\prime}\right)\right),
\end{aligned}
$$

where the valence quark distributions are defined by $q_{v} \equiv q-\bar{q}$. Since the number of valence quarks in a hadron does not change, we obtain the Gotffried sum rule

$$
S_{G} \equiv S_{G}(0,1)=\frac{1}{3} \quad[Q P M]
$$

provided we make the additional assumption $\int_{0}^{1} d x \bar{d}=\int_{0}^{1} d x \bar{u}$, as would be expected in the simple quark-parton model (QPM).

The early experimental data for $S_{G}(x, 1)(B \circ d+73)$ did, in fact, suggest a value lower than $1 / 3$, but with errors large enough to be consistent with it. However, armed with the theoretical expectation of SU(2) flavour symmetry, most authors believed that $S_{G}$ would tend to $1 / 3$ as the accuracy of the data improved. To the surprise of many, the recent, accurate determination of $S_{G}$ by the NMC appears to support the idea that $\bar{u} \neq \bar{d}$ (Ama+91). Neglecting nuclear effects, the NMC found 


$$
S_{G}\left(x_{\min }, 1\right)=0.229 \pm 0.0157
$$

where $x_{\min }=0.004$. The present value which includes an extrapolation to $x=0$, is $S_{G}=0.235 \pm 0.026$ (Arn+94), but this may be lowered a further $4-10 \%$ by shadowing (Zol 92, MT 93a). The most natural explanation for the smaller than expected value of $S_{G}$ is that $\bar{d}(x) \neq \bar{u}(x)$. The value quoted above would imply that $\int_{0}^{1} d x(\bar{d}(x)-\bar{u}(x))=0.15 \pm 0.06$.

The meson-cloud model offers a simple, and at first sight convincing, explanation. Indeed, as we noted earlier the model had already been used to predict $\bar{d}>\bar{u}$ (Tho 83). As discussed above, one can relate the proton and neutron structure function difference to the quark distribution function of the proton only. As we have seen, the physical proton has a relatively large $\pi^{+}$-neutron component in the Fock space, but a much smaller $\pi^{-}-\Delta^{++}$component (the effect of which is further reduced by the $\Delta^{0}-\pi^{+}$contribution). Therefore the Sullivan process immediately gives a surplus of $\bar{d}$-antiquarks. The realistic meson cloud model explains the observed effect nearly quantitatively. This explanation indicates that the derivation of the GSR is due to deep inelastic scattering from the (isovector) meson cloud of the nucleon.

This, however, is difficult to understand if we go back to the original formula equ. (4.2). Here the cloud of $\pi^{+}$and $\pi^{-}$mesons enters symmetrically, so one expects that their effect should drop out. As we will see in the following, a calculation in terms of the meson cloud does give the correct result as far as the (integrated) Gottfried sum rule is concerned. If, however, one is interested in the $x$-distribution (i.e. the functional dependence of the rhs of equ. (4.2)) one has to start with equ. (4.2). The underlying physics is slightly more subtle than that discussed above. To explain it, we first consider a simplified model involving only bare nucleons and pions:

$$
|N\rangle_{p h y}=Z^{1 / 2}|N\rangle_{\text {bare }}+\sum_{i} \alpha_{i}\left|\pi^{(i)}\right\rangle\left|N_{i}\right\rangle_{b a r e}
$$


Within this model we obtain the following contributions for $F_{2}^{\mu p}$ and $F_{2}^{\mu n}$ from the processes shown in Fig. 3.1

$$
\begin{aligned}
& F_{2}^{\mu p}(x)=Z \widetilde{F}_{2}^{p}(x)+P_{p \pi \%} \widetilde{F}_{2}^{p}+P_{p \pi \%} F_{2}^{\pi^{0}}+P_{n \pi \pi^{+} / p} \widetilde{F}_{2}^{n}+P_{n \pi / p} F_{2}^{\pi^{+}}, \\
& F_{2}^{\mu n}(x)=Z \widetilde{F}_{2}^{n}(x)+P_{n \pi \% / n} \widetilde{F}_{2}^{n}+P_{n \pi \% / n} F_{2}^{\pi^{0}}+P_{p \pi / n} \widetilde{F}_{2}^{p}+P_{p \pi / n} F_{2}^{\pi^{-}} .
\end{aligned}
$$

$Z$ is the probability to find a 'bare' nucleon, $\widetilde{F}_{2}^{p}$ and $\widetilde{F}_{2}^{n}$ are the structure functions of the 'bare' proton and neutron, respectively, and $\widetilde{F}_{2}^{\pi}$ is the pion structure function. We also used the following shorthand notations:

$$
\begin{aligned}
& P_{N \pi / N} \widetilde{F}_{2}^{N} \equiv \int_{0}^{1-x} d y f_{N \pi}(y) F_{2}^{N}\left(\frac{x}{1-y}\right) \\
& P_{N \pi / N} F_{2}^{\pi} \equiv \int_{x}^{1} d y f_{\pi N}(y) F_{2}^{\pi}\left(\frac{x}{y}\right)
\end{aligned}
$$

Using charge-symmetry we obtain for the difference of the proton and neutron structure function

$$
\begin{aligned}
F_{2}^{\mu p}(x)-F_{2}^{\mu n}(x) & =\left(Z+P_{p \pi \% p}-P_{n \pi / p}\right)\left(\widetilde{F}_{2}^{p}(x)-\widetilde{F}_{2}^{n}(x)\right) \\
& =\left(Z+P_{p \pi \% p}+P_{n \pi+/ p}-2 P_{n \pi^{+} / p}\right)\left(\widetilde{F}_{2}^{p}(x)-\widetilde{F}_{2}^{n}(x)\right) .
\end{aligned}
$$

From equs. (4.12) one immediately realizes, that, indeed, the pion structure function does not appear. If one divides equ. (4.12a) by $x$ and integrate over $x$ one obtains:

$$
\int \frac{d x}{x}\left(F_{2}^{\mu p}(x)-F_{2}^{\mu n}(x)\right)=\frac{1}{3}-2 \int_{0}^{1} \frac{d x}{x} \int_{0}^{1-x} d y P_{n \pi^{+} / p}(y) \frac{x}{1-y}\left(\widetilde{u}_{v}\left(\frac{x}{1-y}\right)-\widetilde{d}_{v}\left(\frac{x}{1-y}\right)\right),
$$


where $\widetilde{u}_{v}$ and $\widetilde{d}_{v}$ are the valence distributions of the 'bare' proton. Here we have used the fact that in our simplified model the probabilities of finding a bare proton, a neutron with a $\pi^{0}$ and a neutron with a $\pi^{+}$add up to unity, which gives the well known factor $1 / 3$ of the GSR. Deviations from this expected value are proportional to the neutron $-\pi^{+}$admixture, and the proton $-\pi^{-}$admixture in the neutron, respectively. Moreover, one also realizes that the difference in the $x$-distribution is proportional to the valence quark distribution in the (bare) proton. As mentioned already, as long as one is interested in the integrated value of the GSR both formulations given here are equivalent (SS 93).

If one is interested in the $x$-distribution, equ. (4.13) has to be used. Generalizations of equ. (4.13) to include $\Delta$, vector mesons and strange hadrons are straightforward. The result is that only the admixture of isovector-mesons gives rise to a deviation from the GSR. An additional small contribution comes from strange baryons and mesons (HSB 91, TM 93, HSS 96). Calculations within meson models of different stages of sophistication have been performed by many authors. Their results essentially agree with the experimental finding. The $x$-distribution $F_{2}^{p}(x)-F_{2}^{n}(x)$ was calculated first by Melnitchouk et al. (MTS 91, TM 93), who also considered the effect of Pauli-blocking. Their results are shown in Fig. 4.6, where TOPT in the IMF was used with a dipole formfactor (with $\Lambda=700 \mathrm{MeV}$ ). $P$ denotes the fraction of the valence quark normalisation associated with a four-quark bag state spectator to the hard collision (ST 89). The most noticeable consequence of the meson cloud is a decrease in the peak value at $x \sim 0.3$, which indicates a change in the usual regime of valence distributions. The same effect can be seen in Fig. 4.3 where the results of ref. ( $\mathrm{Hol} \mathrm{95)}$ ) are shown. There only the effects of the meson cloud were considered. The various contributions which give rise to the violation of the Gottfried sum rule and to the asymmetry of the sea quark distribution are given in Fig. 4.7 and Table 4.3. In both cases one notices corrections coming from the $\Delta$-configurations. 


\subsection{Drell-Yan-Processes and $\bar{u}-\bar{d}$ Asymmetry}

The Drell-Yan (DY) process (DY 71) involves the electromagnetic annihilation of a quark (antiquark) from the incident hadron $A$ with an antiquark (quark) in the target hadron $B$. The resultant virtual photon materializes as a dilepton pair $\left(\ell^{+} \ell^{-}\right)$ with muons being the pair most readily detected in experiments. This process is schematically shown in Fig. 4.8. The cross section for the DY process can be written as

$$
\frac{d \sigma^{A B}}{d x_{1} d x_{2}}=\frac{4 \pi \alpha^{2}}{9 s x_{1} x_{2}} K\left(x_{1}, x_{2}\right) \sum_{f} e_{f}^{2}\left[q_{A}^{f}\left(x_{1}\right) \bar{q}_{B}^{f}\left(x_{2}\right)+\bar{q}_{A}^{f}\left(x_{1}\right) q_{B}^{f}\left(x_{2}\right)\right]
$$

where $s$ is the square of the center-of-mass energy and $x_{1}$ and $x_{2}$ are the longitudinal momentum fractions carried by the quarks of flavour $f$. The functions $q_{A}^{f}\left(x_{1}\right)\left(\bar{q}_{A}^{f}\left(x_{1}\right)\right)$ and $q_{B}^{f}\left(x_{2}\right)\left(\bar{q}_{B}^{f}\left(x_{2}\right)\right)$ are the (anti-)quark distributions of the beam and target, respectively. The factor $K\left(x_{1}, x_{2}\right)$ accounts for the higher-order QCD corrections that enter the process. Its value over the kinematic range where experiments are carried out is typically 1.5 . The values of $x_{1}$ and $x_{2}$ are extracted from experiment via

$$
M^{2}=s x_{1} x_{2} \approx 2 P_{\ell^{+}} P_{\ell^{-}}\left(1-\cos \theta_{\ell^{+} \ell^{-}}\right)
$$

where $M$ is the mass of the dilepton pair, $P_{\ell^{+}}$and $P_{\ell^{-}}$are the laboratory momenta of the leptons, and $\theta_{\ell^{+} \ell^{-}}$is the angle between their momentum vectors. The total longitudinal momentum of the lepton pair $\left(P_{\ell^{+}}+P_{\ell^{-}}\right)_{L}$ fixes $x_{1}-x_{2}$ via

$$
x_{1}-x_{2} \equiv x_{F}=\frac{2\left(P_{\ell^{+}}+P_{\ell^{-}}\right)_{L}}{s}-1
$$


In order to avoid spurious contributions to the DY yield from vector meson decays, all measurements are made for $M>4 \mathrm{GeV}$, and the region $9 \leq M \leq 11$ $\mathrm{GeV}$ is excluded to avoid the $Y$ resonances.

The absolute value of the Drell-Yan cross section is biased by the uncertainty in extrapolating from time-like to space-like values of $Q^{2}$ when relating the Drell-Yan process with deep-inelastic scattering which involves the factor $K$ (see equ. 4.14). In order to avoid the uncertainty it is desirable to consider ratios (Ald+90, SSG 94) rather than absolute cross sections.

Drell-Yan processes have been proposed to measure the $\bar{u}-\bar{d}$ asymmetry directly. In ref. (Szc+ 96) the various experimental possibilities have been investigated to test their sensitivity. In Fig. 4.9 we show the differential cross section, $M^{3} \frac{d^{2} \sigma}{d x p d M}$ for dilepton production in $p+d$ collision. The full line is the result of the meson cloud model discussed in section 4.2, with an asymmetric sea. In order to test the sensitivity to the $\bar{d}-\bar{u}$ asymmetry, a symmetrized sea distribution and a phenomenological distribution with symmetric $\bar{u}, \bar{d}$ sea have been also used in the calculation. Although there is some sensitivity to the $\bar{d}-\bar{u}$ asymmetry, it can be easily compensated by a slightly different normalization factor.

The present experimental data for the Drell-Yan processes in elementary nucleon-nucleon collisions suffer from rather low statistics. Therefore, at present, one is forced to compare a theoretical calculation with the proton-nucleus experimental data. In first approximation the cross section for the production of the dilepton pairs in proton-nucleus scattering can be expressed in terms of the elementary $p p$ and $p n$ processes as

$$
\sigma_{p A}^{D Y}=Z \sigma_{p p}^{D Y}+N \sigma_{p n}^{D Y}
$$

It has been shown (Ald+90, SSG 94) that the ratio of the cross section for the scattering of protons from the nucleus with $N-Z \neq 0$ to that from an isoscalar target such as deuterium is sensitive to the $\bar{d}_{p}(x)-\bar{u}_{p}(x)$ difference. These ratios have been measured by the E772 Collaboration at FNAL (Ald+ 90) for carbon, calcium, 
iron and tungsten targets. Neglecting nuclear effects, elementary algebra leads to the following result for the ratio:

$$
R_{D X}=\frac{2 \sigma^{D Y}(p+A)}{A \sigma^{D Y}(p+d)}=\frac{2 Z}{A}+\frac{N-Z}{A} \frac{2 \sigma^{p n}\left(x_{1}, x_{2}\right)}{\sigma^{p p}\left(x_{1}, x_{2}\right)+\sigma^{p n}\left(x_{1}, x_{2}\right)^{\prime}}
$$

where $Z, N, A$ are numbers of protons, neutrons and the atomic number, respectively. In the large $x_{2}$ (target) limit the ratio takes a very simple form (McG+ 92):

$$
R_{D X}(x)=1+\frac{N-Z}{A} \frac{\Delta(x)}{\bar{u}(x)+\bar{d}(x)^{\prime}} \text { with } \Delta(x)=\bar{d}(x)-\bar{u}(x)
$$

showing that the Drell-Yan processes with non-isoscalar targets are relevant for the issue of the asymmetry. Here $\Delta(x)$ is defined as

$$
\bar{u}(x)=\bar{q}(x)-\frac{1}{2} \Delta(x) \text { and } \bar{d}(x)=\bar{q}(x)+\frac{1}{2} \Delta(x)
$$

The experimental ratios are consistent with symmetric quark distributions (Ald+90, McG+92) (see Fig. 4.10). Moreover, using asymmetric quark distribution functions (solid and dashed lines) has a rather small effect on the ratio. This renders those data useless for establishing the asymmetry. The ratio obtained with the recent $\operatorname{MSR}(A)$ quark distributions (MSR 94) almost coincides with the result of the meson cloud model ( $\mathrm{Hol}+96)$. As seen from the figure these ratios do not provide a sensitive enough test.

The idea of the $\bar{u}-\bar{d}$ asymmetry is not new. It was considered a decade ago by Ito et al. (Ito 81 ) as a possible explanation for the slope of the rapidity distribution of dilepton production in the proton-PT collision at Fermilab. An alternative interpretation stimulated by the discovery of the EMC effect invoked the enhancement of the nuclear sea in PT with respect to a collection of free nucleons (ET 84). Ito et al. suggested to analyze the logarithmic derivative of the rapidity distribution 


$$
S(\sqrt{\tau})=\left.\frac{d}{d y} \ln \frac{d^{2} \sigma}{d M d y}\right|_{y=0} \text { with } \tau=x_{1} x_{2}
$$

where $y=\ln \left(x_{1} / x_{2}\right) / 2$ is the rapidity. This quantity also possesses the desired property of being independent of the $K$-factor. In terms of the quark distributions the slope can be expressed as product of valence and sea distributions. Therefore the rapidity slope is a quantity which is sensitive not only to the $\bar{u}-\bar{d}$ asymmetry but also to valence quark distributions. In Fig. 4.11 we display the slope of the rapidity distribution calculated with different quark distributions. The solid line is the result of the meson model (Szc+96). The dotted line is the result obtained with the Owens parameterization (Owe 91) of the quark distributions, the dashed line was obtained with the recent $M R S(A)$ parameterization (MSR 94) with $\bar{u}-\bar{d}$ asymmetry and the dash-dotted line was obtained with $M R S\left(S_{0}^{\prime}\right)$ (MSR 93) (symmetric) distribution. Fig. 4.10 clearly demonstrates that the asymmetry is not the only ingredient and a reasonable description of the experimental data can be obtained with both flavour symmetric and asymmetric distributions.

A quantity which can be extracted almost directly from experiment is

$$
A_{D Y}\left(x_{1}, x_{2}\right)=\frac{\sigma_{p p}\left(x_{1}, x_{2}\right)-\sigma_{p n}\left(x_{1}, x_{2}\right)}{\sigma_{p p}\left(x_{1}, x_{2}\right)+\sigma_{p n}\left(x_{1}, x_{2}\right)}
$$

which we will call Drell-Yan asymmetry. In equ. (4.21) $\sigma_{p p}$ and $\sigma_{p n}$ are the cross sections for dilepton production in proton-proton and proton-neutron scattering. The Drell-Yan asymmetry (4.21) can be expressed in terms of the quantity $\bar{q}$ and $\Delta$, introduced in equ. (4.19a)

$$
A_{D Y}\left(x_{1}, x_{2}\right)=\frac{\left[u\left(x_{2}\right)-d\left(x_{2}\right)\right]\left[3 \bar{q}\left(x_{1}\right)-5 / 2 \Delta\left(x_{1}\right)\right]-\left[4 u\left(x_{1}\right)-d\left(x_{1}\right)\right] \Delta\left(x_{2}\right)}{\left[u\left(x_{2}\right)+d\left(x_{2}\right)\right]\left[5 \bar{q}\left(x_{1}\right)-3 / 2 \Delta\left(x_{1}\right)\right]+\left[4 u\left(x_{1}\right)+d\left(x_{1}\right)\right] 2 \bar{q}\left(x_{2}\right)} \text {. }
$$


In the case of a flavour symmetric sea $(\Delta=0)$ it is natural to expect that $A_{D Y}>0$, since $u>d$. However, the sign of $A_{D Y}$ can be reversed by increasing the flavour asymmetry of the proton sea $(\Delta=0)$.

Two dimensional maps of the Drell-Yan asymmetry as a function of $x_{1}$ and $x_{2}$ are shown in the form of the contour plots in Fig. 4.12. The different maps were obtained with the Owens parameterization (OWE 91) (left-upper corner), symmetric $\operatorname{MRS}\left(S_{0}^{\prime}\right)$ (MSR 93) (right-upper corner), the new $M R S(A)$ (MRS 94) with the $\bar{u}-\bar{d}$ asymmetry built in (left-lower corner) and the prediction of the meson cloud model (Szc+ 96) (right-lower corner). The result obtained with the Owens (symmetric) parameterization and symmetric $M R S\left(S_{0}^{\prime}\right)$ parameterization are quite similar. This clearly demonstrates that the asymmetry $A_{D Y}$ is the desired quantity - insensitive to the valence quark distributions. It is also worth noting here that $A_{D Y}$ is positive in the whole range of $\left(x_{1}, x_{2}\right)$. How the $\bar{u}-\bar{d}$ asymmetry influences $A_{D Y}$ is shown in two lower panels. It is very promising that $A_{D Y}$ obtained with the asymmetric quark distributions (lower panels) differs considerably (please note the change of sign in the lower panels) from the result obtained with the symmetric distribution (upper panels) and this should make an unambiguous verification of the flavour asymmetry of the sea quarks possible. It is not random in our opinion that the result obtained within the meson cloud model is very similar to that obtained from the parameterization fitted to different experimental data. We stress, in this context, that $A_{D Y}$ calculated in the meson cloud model is fairly insensitive to the quark distributions in the bare nucleons (baryons). It is primarily sensitive to the $\bar{u}-\bar{d}$ asymmetry which is fully determined by the quark distributions in the pion (and other isovector mesons), taken here from the pion-nucleus Drell-Yan process. In this analysis it has been assumed that the quark distributions in other mesons are related to those for the pion via $S U(3)_{f}$ symmetry.

Following the suggestion of Ellis and Stirling, the NA51 Collaboration at CERN has recently measured the $A_{D Y}$ asymmetry along the $x_{1}=x_{2}$ diagonal (Bal+ 94). Due to low statistics, only $A_{D Y}$ at low $x=x_{1}=x_{2}$ was obtained. In Fig. 4.13 we show their experimental result (one experimental point) together with the results obtained with different quark distributions. The meaning of the lines here is the same 
as in Fig. 4.10. The result denoted as MCM, obtained within the meson cloud model (Hol 95, Hol+ 96) essentially without free parameters, nicely agrees with the experimental data point. In order to better understand the result, and the relation to the $\bar{u}-\bar{d}$ asymmetry, let us express the cross sections in equ. (4.21) in terms of the quark distributions. Assuming proton-neutron isospin symmetry and taking $x_{1}=x_{2}=x$, as for the NA51 experiment, one gets in terms of the quark distributions in the proton

$$
A_{D Y}=\frac{5(u-d)(\bar{u}-\bar{d})+3(u \bar{u}-d \bar{d})}{5(u+d)(\bar{u}+\bar{d})+3(u \bar{u}-d \bar{d})+4(s \bar{s}+4 c \bar{c})}
$$

Let us consider first the case $\bar{u}=\bar{d}$. For a crude estimate one may neglect sea-sea terms (important at small $x$ only) and assume $u_{v a l}(x)=2 d_{v a l}(x)$, which leads to $\dot{A}_{D Y}=1 / 11>0$. The same crude estimate in the case of an asymmetric sea in conjunction with the decomposition equ. (4.19a) yields

$$
A_{D Y}=\frac{-19 \Delta+6 \bar{q}}{-9 \Delta+66 \bar{q}}
$$

This demonstrates a strong sensitivity to both the $\bar{d}-\bar{u}$ asymmetry and to the absolute normalization of the sea. The lack of dependence on the valence quark distributions in the approximate expression equ. (4.24) suggests a weak dependence in the exact formula, equ. (4.23). The negative value obtained by the NA 51 experiment, $A_{D Y}=-0.09 \pm 0.02 \pm 0.025$, automatically implies $\bar{d}>\bar{u}$ - at least for the measured $x=0.18$ (provided that the proton-neutron isospin symmetry violation is small(!)) (ST 96). The data point of the NA51 group is so far the most direct evidence for the flavour asymmetry of the sea quarks, which is explicitly shown in Fig. 4.14. There $A_{D Y}$ has been translated into the ratio $\bar{u}(x) / \bar{d}(x)$. The $x$ dependence of the asymmetry is awaiting further experiments. It is expected that the new experiment planned at Fermilab (Gar+92) will be very useful in this respect and will provide the $x$ dependence of the $\bar{u}-\bar{d}$ asymmetry up to $x=0.4$. It should therefore shed new light on the microscopic structure of the nucleon. The meson- 
cloud model gives definite predictions for the asymmetry awaiting future experimental verification.

\subsection{Polarized Semi-Inclusive Deep-Inelastic Scattering}

Despite the various phenomenological successes of the meson cloud model discussed so far, it is important to look for further experimental evidence pointing unambigously to the existence of a pion and kaon cloud in high energy reactions. Melnitchouk and Thomas (MT 95) have focussed on the semi-inclusive production of polarized $\Delta^{++}$baryons from a polarized proton, $e \vec{p} \rightarrow e^{\prime} \vec{\Delta}^{++} X$ and of a polarized $\Lambda$ from a polarized proton $e \vec{p} \rightarrow e^{\prime} \vec{\Lambda} X$. They suggested experiments at CEBAF and HERMES which could distinguish between the meson-exchange mechanism and parion fragmentation. In Fig. 4.15 the polarization asymmetry $\left(\sigma^{+}-\sigma^{-}\right) / \sigma_{\text {tot }}$ is shown for CEBAF and HERMES kinematics. The two curves represent extreme cases, in which $\Delta^{\prime} s$ are produced entirely via pion emission or diquark fragmentation. In reality, the ratio of polarization cross sections will be some average of the curves in Fig. 4.15. The amount of deviation from the parton model curve will indicate the extent to which the pion-exchange process contributes.

In a similar way, leptoproduction of polarized $\Lambda$ hyperons from polarized protons can be used to test the relevance of a kaon cloud in the nucleon. The advantage of detecting $\Delta^{\prime} s$ in the final state, as compared with $\Delta$ baryons, lies in the fact that the $\Lambda$ is self-analyzing. It has also been suggested (MT 95) that measurement of the polarization of the $\Lambda$ in the target fragmentation region could discriminate between models of the spin content of the nucleon in which a large fraction of the spin is carried either by (negative polarized) strange quarks or (positively polarized) gluons. The latter would imply a positive correlation of the target proton and $\Lambda$ spins, while the spin projection of the $\Lambda$ along the target polarization axis should be negative in the former model. (Similar effects would also be seen in the reaction $\bar{p} p \rightarrow \bar{\Lambda} \Lambda$ (Hai+ 92, AEK 95). The present data on semiinclusive, nonpolarized $\Lambda$ 's, however, indicate that the $\Lambda$ and $\Sigma$ hyperon admixtures in the proton wave function might be small (see the discussions in sects. 4.1 and 4.2). The polarization asymmetry for $\Lambda$-production is shown in Fig. 4.16. In 
first approximation the probability of forming a $\Lambda^{\uparrow}$ and $\Lambda^{\downarrow}$ is equal in the parton fragmentation process and the asymmetry is zero. Therefore the observation of a large polarization asymmetry in the large $-\zeta$ region of target fragmentation will be evidence for a kaon-exchange fragmentation mechanism.

\subsection{Exclusive Electroproduction of Pions}

It has been suggested that the spin averaged splitting functions discussed above may be directly measurable in exclusive electroproduction of pions above the resonance region. This claim is based on the observation (SZ 95) that, for the photon virtuality $Q^{2}$ greatly exceeding the meson and baryon virtualities $k^{2}$, the differential cross section for the reaction $e p \rightarrow e M B$ is dominated by the nucleon pion pole diagram which factorizes

$$
\frac{d \sigma}{d x d Q^{2}} \sim f_{\pi N}(x) F_{\pi N}^{2}\left(Q^{2}\right)
$$

Here, $F_{\pi(N)}\left(Q^{2}\right)$ is the on-mass-shell electromagnetic form factor of the struck pion (nucleon).

. In (SZ 95) the splitting functions are interpreted as the densities of (nonperturbative) partons (mesons and baryons) of the physical nucleon and it is suggested that one could study them like parton densities in inclusive DIS. Indeed, from equ. (4.25) it follows that in exclusive one-pion electroproduction the photon of high virtuality, $Q^{2}$, probes the density of mesons and/or baryons in the proton at the value of the light-cone Sudakov variable equal to the Bjorken variable $x$. Factorization implies a possibility of separate analysis of the light-cone mesonbaryon density functions and the electromagnetic form factors of mesons and baryons.

More accurately, the decomposition of the differential cross section of the exclusive reaction $e p \rightarrow e \pi^{+} n$ in terms of the longitudinal and transverse cross sections reads (SZ 95). 
$\frac{d \sigma\left(e p \rightarrow e \pi^{+} n\right)}{d x d Q^{2} d k_{\perp}^{2}}=2 K_{L}\left(x, Q^{2}\right) f_{\pi N}\left(1-x, k_{\perp}^{2}\right) F_{\pi}^{2}\left(Q^{2}\right)+2 K_{r}\left(x, Q^{2}\right) f_{\rho N}^{T}\left(1-x, k_{\perp}^{2}\right) \frac{1}{8} Q^{2} F_{\rho \pi}^{2}\left(Q^{2}\right)$

where $K_{L}$ and $K_{T}$ in (4.26) are the kinematical factors.

In equ. (4.26) $F_{\pi}\left(Q^{2}\right)$ is the on-shell charge form factor of the pion. It is worth recalling that in the light-cone parton model the condition $Q^{2} \gg k^{2}$ guarantees the on-shellness of partons (SZ 95). The second (transverse) term in (4.26) arises from the $\rho^{+}-\pi^{+}$radiative transition which is under the control of the form factor $F_{\rho \pi}\left(Q^{2}\right)$. This is a magnetic dipole (M1) transition - hence the enhancement factor, $Q^{2}$, in the corresponding term in the electroproduction cross section (4.26).

The density of the transversely polarized $\rho$-mesons in the proton, $f_{\rho \mathrm{N}}^{T}$, can easily be obtained ( $S Z$ 95). It is worth noticing that the $\rho$-pole dominated transverse cross section is expected to be an increasing function of $k_{\perp}^{2}$ at small $k_{\perp}$. This effect is due to the strong $k_{\perp}$-dependence of the tensor term

$$
\frac{f}{4 m_{N}} \bar{\psi} \sigma_{\mu \nu} \psi\left(\partial^{\mu} \rho^{\nu}-\partial^{v} \rho^{\mu}\right)
$$

in the $\rho N N$ interaction, which generates terms proportional to $k_{\perp}^{2}$ and $k_{\perp}^{4}$ in $f_{\rho \mathrm{N}}^{T}$. Observation of this phenomenon is crucial for understanding the mechanism of exclusive electroproduction.

The possibility to detect neutral mesons makes the reaction $e p \rightarrow e \pi^{0} p$ particularly interesting as it is free of the pion-pole term and at high $Q^{2}$ the baryon pole term is suppressed by extra powers of $1 / Q^{2}$. Then the differential cross section reads

$$
\frac{d \sigma\left(e p \rightarrow e \pi^{o} p\right)}{d x d Q^{2} d k_{\perp}^{2}}=K_{T}\left(x, Q^{2}\right) f T_{\omega N}\left(1-x, k_{\perp}^{2}\right) \frac{1}{8} Q^{2} F_{\omega x}^{2}\left(Q^{2}\right)
$$


There are two main reasons for the $\omega$-pole dominance. First of all the $\omega \pi y$ coupling constant, absorbed in the radiative transition form factor, $F_{\omega \pi}^{2}\left(Q^{2}\right)$, is numerically large. The extra enhancement is due to the factor, $Q^{2}$, typical of the magnetic dipole interaction (SZ 95).

This observation makes the idea of the precision measurement of the $Q^{2}$ dependence of the cross section of the reaction $p\left(e, e^{\prime} \pi^{0}\right) p$ especially appealing, since it provides us with opportunity of direct measurement of the vector meson cloud of the nucleon. Furthermore, as has been emphasized in (SZ 95), the early onset of the parton model regime enables one to study the helicity non-conserving form factors $F_{\rho \pi}\left(Q^{2}\right), F_{\omega \pi}\left(Q^{2}\right)$ in the substantially non-perturbative region and, what is more important, to retrace the onset of the $p Q C D$ regime at a very high $Q^{2}$ as welis.

In a recent detailed analysis (PP 92), it has been also suggested that measurements of fast pions in the final state, in coincidence with the final electron, could be sensitive to a pionic component of the nucleon. Extending the exclusive analysis of Güttner et al. (Güt+ 84) in the IMF, Pirner and Povh (PP 92) work within a constituent quark picture in which the probability to find a pion in the nucleon is expressed in terms of the pion distribution function inside a constituent quark.

The differential pion-production cross section for the "leading pion" (integrated over transverse momenta) is written as

$$
\begin{aligned}
\frac{d \sigma}{d x d y d z} \propto \quad & A(x, y, z)+B(x, y, z) F_{\pi}\left(Q^{2}\right) \\
& +C\left(x, y, z^{\prime}\right) F_{\pi}^{2}\left(Q^{2}\right),
\end{aligned}
$$

where $z=E_{\pi} / v$ is the fraction of the photon's energy carried by the pion, and where the $A$ and $B$ terms describe soft and hard fragmentation, respectively. The function $C$ reflects coherent scattering from the pion cloud of the constituent quark. Each term in equ. (4.28) gives a characteristic $Q^{2}$-dependence, namely $\log Q^{2}, 1 / Q^{2}$ and $1 / Q^{4}$, respectively. To isolate the coherent scattering from the pion one therefore 
has to restrict oneself to the region of not too high $Q^{2}$, where the form factor suppression has not yet eliminated the pion signal.

A useful observation in this analysis is that each of the three processes has a quite distinct $z$-dependence. The hard-fragmentation process gives a differential cross section which is constant in $z$, and is important in the intermediate $z$ region $(0.6 \leq z \leq 0.8)$. The soft fragmentation mechanism is dominant at small $z, z \leq 0.6$, but dies out rather rapidly at larger $z$. This fact may enable one to detect the pionexchange process, which dominates the region $0.8 \leq z \leq 1$, where it predicts a contribution that is several times larger than the constant- $z$, hard fragmentation mechanism. The conclusion that the pion-exchange process is dominant is consistent with our previous results.

The authors argue, however, that what they suggest is the measurement of the meson cloud of the constituent quark and not the meson cloud of the nucleon. In their view the meson cloud of the nucleon has already been determined from the pion-electroproduction data on the proton where only a $3 \%$ admixture of $n \pi^{+}$in the bare proton was found (Güt+ 84). This should be compared with the corresponding value in Table 4.1, where the $\pi^{+} n$ admixture is $12 \%$ (and $6 \%$ is $\pi^{0} p$ ). This crucial difference in the concept of the meson cloud will be decided very soon experimentally by the ZEUS collaboration at HERA, which is installing a forward neutron calorimeter.

\subsection{Meson-Cloud Effects on the Spin-dependent Properties of the Nucleon}

The spin structure functions (equ. 2.10) of the proton and neutron are related by the Bjorken sum rule, which is a rigorous prediction of $Q C D$

$$
\int_{0}^{1} d x\left[g_{1}^{p}(x)-g_{1}^{n}(x)\right]=\frac{1}{6} g_{A} / g_{v} .
$$

It was first derived using current algebra (Bjo 66) where one can show that the integrated value of $g_{1}^{N}(x)$ is given (in the Bjorken limit) by the matrix-element 


$$
\Gamma_{N}=\int_{0}^{1} d x g_{1}^{N}(x)=\frac{1}{2}\left\langle N \uparrow\left|\bar{\Psi}(0) Q^{2} \gamma_{3} \gamma_{5} \Psi(0)\right| N \uparrow\right\rangle .
$$

Using the relation

$$
\frac{1}{2} Q^{2}=\frac{1}{12}\left[\lambda_{3}+\frac{1}{\sqrt{3}} \lambda_{8}+2 \sqrt{\frac{2}{3}} \lambda_{0}\right]
$$

where the $\lambda$ 's are the usual Gell-Mann matrices, one obtains what for the proton is called the Ellis-Jaffe Sum Rule (EJ 74).

$$
\Gamma_{p}=\frac{1}{6}\left[I_{0}+I_{8}+I_{3}\right]
$$

In terms of the standard SU(3) amplitudes, $F$ and $D$, for the baryon semileptonic decays, the two flavor octet amplitudes are given as

$$
I_{3}=\frac{1}{2}\left\langle P \uparrow\left|\bar{\Psi} \gamma_{3} \gamma_{5} \lambda_{3} \Psi\right| P \uparrow\right\rangle=\frac{1}{2}(F+D),
$$

and

$$
I_{8}=\frac{1}{2 \sqrt{3}}\left\langle P \uparrow\left|\bar{\Psi} \gamma_{3} \gamma_{5} \lambda_{8}\right| P \uparrow\right\rangle=\frac{1}{2}(F-D) .
$$

The flavor singlet amplitude is given as

$$
I_{0}=\sqrt{\frac{2}{3}}\left\langle P \uparrow\left|\bar{\Psi} \gamma_{3} \gamma_{5} \lambda_{0} \Psi\right| P \uparrow\right\rangle
$$

and can be deduced from the experimentally known $\Gamma_{p}$ from equ. (4.32) if one takes $F$ and $D$ from the analysis of the semi-leptonic decays of the baryon octet.

Usually one expresses the Ellis-Jaffe sum rule in terms of the polarized quark distributions 


$$
\begin{aligned}
& \Delta q=\int_{0}^{1} d x\left[q^{\uparrow}(x)-q^{\downarrow}(x)+\bar{q}^{\uparrow}(x)-\bar{q}^{\downarrow}(x)\right], \\
& \Gamma_{p}=\int_{0}^{1} g_{\uparrow}^{p}(x) d x=\frac{1}{2}\left(\frac{4}{9} \Delta u+\frac{1}{9} \Delta d+\frac{1}{9} \Delta s\right) .
\end{aligned}
$$

Correspondingly one expresses the semileptonic decay of the baryon octet in the Cabbibo model, where one assumes that the axial currents responsible for the semi-leptonic decays belong to an SU(3) octet. The diagonal matrix elements of these axial currents in this specific model give the well-known connection to the Ellis-Jaffe sum rule. Note, that in equ. (4.37) the polarization of quarks and antiquarks enter, whereas the Cabbibo model considers only the three valence quarks.

$$
\begin{gathered}
2 g_{A}^{3}=\Delta u-\Delta d=F+D \\
2 \sqrt{3} g_{A}^{8}=\Delta u+\Delta d-2 \Delta S=3 F-D \\
g_{A}^{0}=\Delta u+\Delta d+\Delta s .
\end{gathered}
$$

If one assumes that there is no polarized strangeness contribution, i.e. $\Delta s=0$ as Ellis and Jaffe assumed, $g_{A}^{0}$ is given by equ. (4.40), and one obtains the result of ref. (EJ 74)

$$
\begin{aligned}
& \int g_{1}^{p}(x) d x=\frac{g_{A}}{12}\left(1+\frac{5}{3} \frac{3(F / D)-1}{(F / D)+1}\right), \\
& \int g_{1}^{n}(x) d x=\frac{g_{A}}{12}\left(-1+\frac{5}{3} \frac{3(F / D)-1}{(F / D)+1}\right) .
\end{aligned}
$$

The recent interest in these sum rules originates from the measurements of the spin structure functions of the proton and neutron by the EMC-(Ash+ 89), SMC- 
(Ada+ 94) and SLAC-(Ant+ 93) collaborations. The first measured value of the proton structure function by EMC caused great excitement because it deviated appreciably from the original Ellis-Jaffe prediction and indicated that only a small fraction of the proton spin is carried by quarks. The latest analysis of the newer data by Ellis and Karliner (EK 95) gives (at $Q^{2}=10 \mathrm{GeV}^{2}$ ).

$$
g_{A}^{(o)}=0.31 \pm 0.07,
$$

This is commonly interpreted as meaning that only $1 / 3$ of the spin of the proton comes from the spin of the quarks. While it is not our primary concern here, we stress that such an interpretation is quite incorrect. Because of the axial anomaly in the flavor singlet channel, $g_{A}^{(o)}$ bears no formal relationship to the spin of the proton (BT 94).

In view of the importance of the meson cloud for the Gottfried sum rule violation, one might expect that it could also play an important role for the nucleon spin. Indeed, an early estimate within the one pion exchange model seemed to indicate that meson cloud effects may play a role in resolving the 'spin crisis' (ST 88). Because of the close connection of the Ellis-Jaffe sum rule with semileptonic decays of the octet baryons we will first review the consequences of the meson cloud for these properties. Modifications of the axial-vector coupling constants due to the meson cloud can be quite important, since the corresponding axial-currents are not protected against renormalization due to the meson cloud (BR 79, TMT 82, Kub 85).

\section{A. Semileptonic Decays}

According to our present understanding, the weak semileptonic decays of the octet baryons can be classified into two groups: either a $d$-quark is transformed into a $u$-quark, or an $s$-quark is transformed into a $u$-quark. The matrix elements of the current operators 'responsible' for the semileptonic decays of the baryons belonging to the octet can be parameterized in terms of $q^{2}$-dependent form factors. 


$$
\left\langle B_{1}\left|V_{\mu}+A_{\mu}\right| B_{2}\right\rangle=C \bar{u}_{B_{1}}\left[\begin{array}{ccc}
f_{1}\left(q^{2}\right) \gamma_{\mu} & +i \frac{f_{2}\left(q^{2}\right)}{m_{1}+m_{2}} \sigma_{\mu \nu} q^{\nu} & +\frac{f_{3}\left(q^{2}\right)}{m_{1}+m_{2}} q_{\mu} \\
+g_{1}\left(q^{2}\right) \gamma_{\mu} \gamma^{5} & +i \frac{g_{2}\left(q^{2}\right)}{m_{1}+m_{2}} \sigma_{\mu \nu} q^{\nu} \gamma^{5} & +\frac{g_{3}\left(q^{2}\right)}{m_{1}+m_{2}} q_{\mu} \gamma^{5}
\end{array}\right] u_{B_{2}}
$$

- The factor $C$ here is the Cabibbo factor $\left(\sin \theta_{c}\right)$ or $\left(\cos \theta_{c}\right)$. At low momentum transfer only two terms, $f_{1}$ (vector) and $g_{1}$ (axial vector), are important. It is customary to extract from experiments the ratio $g_{A} / g_{V}=g_{1}(0) / f_{1}(0)$.

Mesonic corrections lead to the renormalization of the axial-vector coupling constants. The vector coupling constants are protected against renormalization by vector current conservation. Mesonic corrections to the axial-vector coupling constants have been taken into account by calculating the loop corrections to the tree level approximation according to

$$
\left\langle N\left|A_{\mu}^{a}\right| N\right\rangle_{\text {dressed }}=Z\left(\begin{array}{rl}
\left\langle N\left|A_{\mu}^{a}\right| N\right\rangle_{\text {bare }} & +\sum_{B_{1} B_{2} M} \Delta f_{\left(B_{1} B_{2}\right) M / N}\left\langle B_{1}\left|A_{\mu}^{a}\right| B_{2}\right\rangle \\
& +\sum_{M_{1} M_{2} B} \Delta f_{\left(M_{1} M_{2}\right) B / N}\left\langle M_{1}\left|A_{\mu}^{a}\right| M_{2}\right\rangle
\end{array}\right)
$$

The polarized splitting functions $\Delta f$ were defined in section 3.6 and $A_{\mu}^{a}$ are the axial current operators. The corresponding diagrams are shown in Fig. 4.17

The formalism outlined in section 3 has been recently applied by two groups to the semileptonic decay of the octet baryons. Zoller (Zol 93) considered corrections due to the admixture of the pseudoscalar octet and the baryon decuplet, whereas Holtmann et al. (HSS 96) included, in addition, the vector meson nonet.

To perform numerical calculations within this model requires the knowledge of the axial coupling constants for the bare octet and decuplet baryons, vector mesons and the transitions octet $\leftrightarrow$ decuplet: The transitions within the baryonic octet are tracitionally parameterized by the so-called anti-symmetric $F$ and symmetric $D$ coupling constants. The axial coupling constant for the transition within the decuplet (H) can be fixed by the relation $2\left\langle\Delta^{++}\left|A_{\mu}^{3}\right| \Delta^{++}\right\rangle=H \cdot 2 m_{\Delta} s_{\mu}$. In analogy one defines 
the coupling constant for the interference diagram octet $\leftrightarrow$ decuplet $(I)$ as $2\left\langle p\left|A_{\mu}^{3}\right| \Delta^{0}\right\rangle=2\left\langle\Delta^{0}\left|A_{\mu}^{3}\right| p\right\rangle=I \cdot 2 \sqrt{m_{N} m_{\Delta}} S_{\mu}$. The matrix elements of the axial-vector current between pseudoscalar mesons vanish. They are, however, finite for vector mesons. Here the structure is analogous to that of the baryonic octet. The corresponding constants are denoted as $F V$ and $D V$. Due to parity-conservation the axial coupling constant $F V$ vanishes. The vector meson $\leftrightarrow$ pseudoscalar meson interference terms have an octet structure analogous to the other cases, with coupling constants called $F I$ and $D I$.

In the SU(6) model, i.e. in the model in which all particles are described by their SU(6) wave functions (Clo 79), the axial coupling constants can easily be calculated

$$
F=\frac{2}{3}, \quad D=1, \quad H=1, \quad I=4 \frac{\sqrt{2}}{3}, \quad F V=0, \quad D V=1, \quad F I=1, \quad D I=0 .
$$

In Table 4.4 various theoretical results obtained with different modelassumption for all possible semileptonic decays are compared with the available data (PDG 02). In the column named 'MC, $S U(6)$ ' the $g_{A}$ 's are shown calculated within the model of Holtmann et al., using the unrenormalized SU(6) axial-vector coupling constants (equ. 4.45). In the column labeled 'MC, SU(3)' the corresponding results are given with $F$ and $D$ fitted to the measured values of the axial-vector coupling constants (the remaining parameters $H, I, D V$ and $F I$ were take at their SU(6) values). For comparison the tree-level (no mesonic corrections) SU(6) model and SU(3) model ( $F$ and $D$ fitted to the experimental data) are also included. The latter is the 'Cabbibo'-model.

The $\chi^{2}$ values presented in the last row for each model give an idea of the fit quality. It is well known that the naive SU(6) model gives a very poor description of the experimental semileptonic decay data. On the other hand, when fitting the $F$ and $D$ parameters an extremely good description of the existing (5!) data can be achieved. It is commonly believed that any correction to the SU(3) model may only destroy this nice agreement. In fact, the inclusion of mesonic corrections with SU(6) axial coupling constant improves the description of the data dramatically 
$\left(\mathrm{X}^{2} / N=4369 \rightarrow \mathrm{X}^{2} / N=8.5\right)$. An additional variation of the $F$ and $D$ parameters can improve the results slightly. As pointed out by Zoller (Zol 93), the agreement with the data of the MCM-model plus SU(6) coupling parameters is comparable with the Cabbibo model. Whereas the latter has two parameters to fit the 5 experimental data, the SU(6)-MCM is completely parameter free, because everything is fixed by previous investigations. On the other hand, it is also clear that the axial coupling parameters derived from the nonrelativistic constituent quark model have to be modified due to the fact that in relativistic quark models an appreciable amount of the proton spin arises from the orbital motion of the 3 quarks (Bog 63, $\mathrm{HI} \mathrm{82).}$

\section{B. The Ellis-Jaffe Sum Rule}

In order to calculate the sum rule within the MCM one has to calculate the three matrix elements $I_{0}, I_{3}, I_{8}$ given in equations 4.33-4.35. The $I_{3}$ and $I_{8}$ are directly connected with the semileptonic decay of the octet baryons. If one assumes that the polarization of the strange sea can be neglected (as done by Ellis and Jaffe (EJ 74)) then $g_{A}^{o}=2 \sqrt{3} g_{A}^{8}$ and therefore $I_{0}$ is also connected with the leptonic decay. The same two versions of the MCM discussed in the previous section have also be applied to the 'spin-problem' of the nucleon. Zoller (Zol 93) considered the baryon and pseudoscalar meson octet and the baryon decouplet. He used SU(6) axial coupling parameters. The polarization of the strange quarks is very small $(\Delta s=0.005$ ), and the fraction of the spin (helicity) carried by the quarks is about $80 \%$. In his model the three major Fock space components are:

$$
|N\rangle_{\text {phys }}=0.568|N\rangle_{\text {bare }}+0.238|\pi N\rangle+0.125|\pi \Delta\rangle
$$

If one used this decomposition in a static model the matrix element of the spin operator $\hat{S}_{z}$ between proton states with $S_{z}=1 / 2$ reads:

$$
\left\langle\hat{S}_{z}\right\rangle_{p} \approx 0.568 * 1 / 2+0.238 *\left(\frac{-1}{6}\right)+0.125 * \frac{5}{6}=0.35
$$


This value should be compared with $1 / 2$. The depolarization effect is due to the spiñ-flip of the $\pi N$ component, whereas the $\pi \Delta$ term tends to retain the proton polarization. In the actual, relativistic case, the spin-flip and non-spin-flip terms nearly cancel each other (see section 3.6) and this results in a vanishing contribution of the $\pi N$ component to $\left\langle\hat{S}_{z}\right\rangle_{p}$. For that reason the corresponding value in the full model is even larger $\left(\left\langle\hat{S}_{z}\right\rangle_{p}=0.4\right)$. This result depends on the cut-off parameters chosen for the meson-baryon vertices. For harder formfactors (Zol 93) more of the proton spin is carried by the meson cloud but on the other hand the axial couplings of hyperons become unacceptible small. The results of Holtmann et al. (Hol+96) are summarized in Table 4.5, which includes the Ellis-Jaffe sum rule for the proton $S_{E J}^{p}$ and the neutron, $S_{E J}^{n}$, the Bjorken sum rule $S_{B}$, the polarization of the strange sea $\Delta s$ and $q_{0}$ (which corresponds $2 *\left\langle\hat{S}_{z}\right\rangle$ ). In the "SU(6)" model the axial

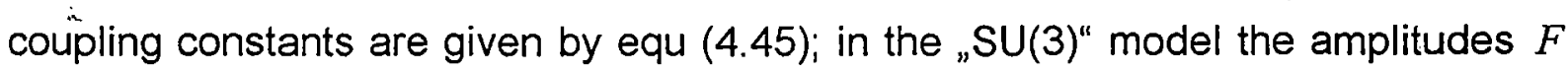
and $D$ also are fitted to the semileptonic decay data. The wave function includes (a) tree level, (b) tree level plus octet baryons and pseudoscalar mesons (oct, ps) (c) as in (b) but also including the decuplet baryons (oct, dec, ps) (d) denoted by "full" includes, in addition, the vector meson octet.

On the tree level in SU(6) all the spin of the proton is due to the spin of the quarks. If one considers the mesonic admixture, $15 \%$ of the proton spin arises from the orbital momentum of mesons and bare baryons. In the SU(3) case, $q_{0}$ is already reduced to 0.5 on the tree level because of the effective $F$ and $D$ amplitudes. Mesonic corrections do not further reduce that value (note: the $F$ and $D$ parameters are refitted). It is important to realize that in the MCM with SU(6) parameters one implicitly assumes that the spin of the "bare" nucleon, delta and adnixed mesons is $100 \%$ quark spin. This, however is not true in relativistic model where nearly $50 \%$ of the nucleon is due to orbital momenta of the quarks (small components of the Dirac wave function). Calculations which include this effect have not yet been done.

The results of the MCM using effective $F$ and $D$ amplitudes resemble very much earlier calculations within the cloudy bag model (ST 88). Also here the 
depolarization due to the $\pi N$-admixture is essentially compensated by the $\pi \Delta$ admixture, so that the originally MIT-bag value of $S_{E J}^{p}=0.18$ is only slightly reduced.

The comparison of the two models also sheds some light on the modeldependence of the Ellis-Jaffe sum rule which relies completely on the Cabbibomodel of semileptonic decays. Whereas these decays can be equally well described in the parameter free MCM-SU(6) model and the 2 parameter SU(3) (Cabbibo) model, the sum rule and the spin content $\Delta q_{0}$ are very different. In particular one knows that the $U_{A}(1)$-anomaly (PP 76, BT 94, Fri 89, SV 90) may play an important role.

\section{MESONS IN THE PROTON AS TARGETS FOR DEEP-INELASTIC SCATTERING}

The experimental determination of the quark structure function of the pion is of crucial importance for our understanding of hadron physics. From chiral symmetry one expects that the pion, as a Goldstone mode, might have a quite different structure compared with other mesons and baryons. Actually, quark-models predict that the pion should be a highly collective object and that this might show up in the structure function - as is the case for collective states in nuclear physics.

Up to now the only feasible method to extract the pion structure function has been the $\pi N$ Drell-Yan production. The disadvantages of this method are that the attainable luminosity is low and that only the valence part of the pion structure function at rather large $x(\geq 0.2)$ can be studied - see however Lon+95. An extension of our knowledge of the pion structure function is possible by using the virtual pions of the meson cloud around the proton as targets in deep inelastic scattering. $\mathrm{Hol}+$ 94): As we have discussed before, these pions arise naturally as a consequence of the pion-nucleon coupling and the interaction of high-energy projectiles like nucleons, pions or leptons with the virtual pion of the $\pi N$ Fock state of the proton. It is a typical stripping reaction, in which the momentum distribution of the spectator nucleon reflects the momentum distribution in the $\pi N$ (meson-baryon) Fock state. 
In Fig. 5.1 a, we show again the $p p$-reaction which has been used in section 4.1 to determine the $\pi^{+}$and $\rho^{+}$Fock states in the proton. It is important to realize that this reaction is in the same kinematic region as the deep-inelastic electron scattering, shown in Figs. $5.1 \mathrm{~b}$ and $5.1 \mathrm{c}$. Therefore once the fluxes (splitting functions) $f_{n \pi+/ p}\left(z, p_{\perp}^{2}\right)$ and $f_{\Delta^{++\pi} \pi / p}\left(z, p_{\perp}^{2}\right)$ are known, one can reverse the "Sullivan processes" and determine the pion and rho structure functions from the semiinclusive production of neutrons. As in the $p p$-reaction we expect that the semiinclusive reactions
(a) $e p \rightarrow e^{\prime} n X$
(b) $e p \rightarrow e^{\prime} \Delta X$,

in the properly chosen kinematical domain, will also be dominated by the pion exchange (MHE 87) mechanism of Fig. 5.1. If this is the case, then the straightforward generalization of equ. (4.1) to semi-inclusive deep inelastic electron scattering is

$$
\begin{aligned}
& \frac{d \sigma\left(e p \rightarrow e^{\prime} n X\right)}{d x d Q^{2} d z d p_{\perp}^{2}}=\frac{2}{3} f_{n \pi^{+} / p}\left(z, p_{\perp}^{2}\right) \\
& x K\left(x, Q^{2}\right) F_{2}^{e \pi}\left(x_{\pi}, Q^{2}\right),
\end{aligned}
$$

where $F_{2 \pi}^{e \pi}\left(x_{\pi}, Q^{2}\right)$ is the structure function of the pion; $x_{\pi}=x /(1-z)$ is the Bjorken variable in the electron-pion deep inelastic scattering, with the obvious kinematical restriction $0<x<1-z$, and $K\left(x, Q^{2}\right)$ is the standard kinematical factor

$$
K\left(x, Q^{2}\right)=\frac{4 \pi \alpha^{2}}{Q^{4}} \frac{1}{x}\left[1-y+\frac{1}{2} y^{2}\right], y=\frac{Q^{2}}{x s}
$$

assuming for the sake of simplicity $2 x F_{1}^{e x}(x)=F_{2}^{e x}(x)$. Knowing all kinematical variables, and trusting the theoretical prediction for $f_{n \pi / p}\left(z, p_{\perp}^{2}\right)$, one can invert equ. (5.2) and determine the pion structure function from the experimentally measured semi-inclusive cross section. In the HERA experiments, one can go down to the 
region of very small $x_{\pi}\left(\geq 10^{-4}\right)$. This will be an enormous expansion of the kinematical region studied compared with the $\pi N$ Drell-Yan experiments, which cannot go much below $x_{\pi} \sim 0.1$ (Sut+92). Furthermore, such a determination of the pion structure function at HERA would allow one to study the scaling violations in the pion structure function in a broad range of $\left(x_{\pi}, Q^{2}\right)$, which is hardly possible in the Drell-Yan experiments.

From the purely experimental point of view, the semi-inclusive reaction $e p \rightarrow e^{t} n X$ is being studied already by the ZEUS collaboration, which has installed a test forward neutron calorimeter (FNC) to complement its leading proton spectrometer (Bha+ 95). This FNC was tested with neutrons from inclusive proton beam-gas interactions, and an excellent agreement between the measured spectra and the pion-exchange predictions was found.

The principal task is to find the kinematical domain in which the semi-inclusive reaction $e p \rightarrow e n X$ is dominated by the pion-exchange contribution. The semiinclusive production of neutrons with $z \sim 0.8$ turns out to be the optimal kinematical domain, and it also corresponds to the domain in which the semi-inclusive cross section is largest. The fluxes for the charge-exchange reactions, $p \rightarrow n$ and $p \rightarrow \Delta^{++}$, as well as the reaction $p \rightarrow p$, are shown in Fig. 5.2. The expected counting rates can be judged by the total number of virtual pions in the nucleon as given in Table $4.1, n_{\pi}(\pi N) \approx 0.18, \quad n_{\pi}(\pi \Delta) \approx 0.06$, which shows that deep inelastic scattering on pions, accompanied by $p \rightarrow n, \Delta$ fragmentation, will have a statistical weighting only one order of magnitude lower than that for ep scattering.

The background to the pure pion exchange comes from interaction with Fock states which contain heavier mesons $M=K, \rho, \omega \ldots$ Evidently, in such states the heavy mesons $M$ will carry a larger fraction of the momentum of the $M N$ state, and the heavy meson exchange will contribute to the spectrum of neutrons at smaller $z$ in comparison with the pion exchange (we do not discuss here the region of $1-z<1$, where the Reggeization of mesons becomes important). In Fig 5.2 the effect of the $\rho$-meson is shown as a dotted curve. Evidently, choosing the region $z \sim 0.7-0.8$, one can eliminate much of the $\rho$-exchange background. A still better separation of the $\pi$ and $\rho$ exchange can be achieved if one compares the $p_{\perp}$ 
distributions for the two mechanisms. As shown in Fig. 5.3 the best way of discriminating between the $\pi$ and $\rho$ contribution is to select only events with $p_{\perp}^{2} \leq 0.1(\mathrm{GeV} / \mathrm{c})^{2}$. Then the relative contribution of the pion exchange is significantly enhanced.

In the suggested mechanism for semi-inclusive neutron production, the differential cross section (5.2) is a product of the universal flux factor, which only depends on $z$, and the structure function $F_{2}^{n}\left(x_{\pi}, Q^{2}\right)$ which is a function of $x_{\pi}=x /(1-z)$. This factorization property allows an important cross check of the model: binning the semi-inclusive cross section data as a function of $z$ should not depend on $x_{\pi}$. Furthermore this $z$-dependence should be identical to the $z$ dependence of the inclusive spectra of neutrons from the hadronic $\rho N$ interactions. Remarkably, the FNC of the ZEUS collaboration enables the latter cross check to be performed in situ, directly comparing the spectra of neutrons from inclusive beamgas interactions and from deep inelastic ep scattering. Such a comparison of the two spectra will allow one to verify that the background contribution to $z \sim 0.7-0.8$, from deep inelastic scattering off the baryonic core, is as small as in hadronic reactions. Reversing the argument, one can determine the $x_{\pi}$ dependence of the pion structure function by changing $x$ at fixed values of $z$ and verifying that the $x_{\pi}$ dependence comes out the same at all values of $z$.

The above discussion is fully applicable to the semi-inclusive production of $\Delta^{++}$. The longitudinal momentum distribution of $\Delta^{++}$is shown in Fig. 2c. As in the $p \rightarrow n$ case, the contributions from the $\pi$ and $\rho$ exchange mechanism are fairly well separated, with the $\pi$-exchange contribution dominating at large $z$. Measuring the $\Delta^{++}$production at HERA will require good experimental resolution of both the proton and $\pi^{+}$resulting from the $\Delta^{++}$decay, which requires multitrack identification of the leading proton spectrometer. The ZEUS collaboration has such a device operating at HERA (ZEU 93). The measurements of $\Delta^{++}$production are important for the direct evaluation of the contribution of the two-step process $p \rightarrow \Delta \rightarrow n \pi$ to the spectrum of neutrons. The $\Delta$ decay background to the spectrum of neutrons is small - isospin 
symmetry considerations imply that the relative contamination of the neutron spectra $n_{\pi}(\pi \Delta) /\left(3 n_{\pi}(\pi N)\right) \approx 0.1$.

It is important to realize that in order to relate $x_{\pi}$, the Bjorken variable in the electron-pion deep inelastic scattering and $z$, the longitudinal momentum fraction carried by the neutron, the basic symmetry for the splitting function (equ. 3.7) has to be fulfilled. As discussed in section 3 this puts stringent restrictions on the form factors in the vertex function which are not fulfilled by the conventional form-factors which depend only on the momentum variable $t$.

\section{CONCLUSION}

On very general grounds the meson cloud must play an important role in the structure of the nucleon. In particular $\pi$-nucleon, $\pi$-delta and $\rho$-nucleon Fock space components constitute nearly $40 \%$ of the nucleon wave function. We have explored the phenomenological consequences of the cloud in a variety of experiments and we have shown that it gives large effects in the nonperturbative regime of $Q C D$. The investigations range from semi-inclusive nucleon-nucleon scattering to polarized and unpolarized DIS, semi-inclusive DIS, exclusive electroproduction of mesons, Drell-Yan experiments and semi-leptonic decays of baryons.

The model so far has several outstanding successes:

I. The initial prediction of the asymmetry of the quark sea $\bar{d}>\bar{u}$ has been confirmed some years later by NMC.

II. After determining the $\pi N$ and $\rho N$ form factors from semi-inclusive $p p$ data, recent calculations quantitatively reproduce (parameter free) the measured $\bar{d}-\bar{u}$ asymmetry. Also the calculated shape of $\bar{u}(x) / \bar{d}(x)$ seems to be well described.

III. Deep-inelastic scattering off the meson cloud explains a significant part of the experimental sea-quark structure functions of the nucleon for $Q^{2} \leq 5 \mathrm{GeV}^{2}$ and $x>10^{-2}$. 
IV. The meson cloud model reproduces the 5 experimentally known data of the semi-leptonic decay of the baryon octet equally as well as the 2 parameter fit of the Cabbibo model.

V. Valence quark structure functions deduced from a model which combines the bag and meson cloud model agree surprisingly well with the phenomenological ones.

Moreover the model makes some definite predictions which may be tested in near future:

(i) The polarization of the strange quark sea in the nucleon is very small. Therefore strange quarks carry a negligibly small fraction of the spin of the nucleon.

(ii) The fraction of the spin of the nucleon which arises from the orbital momentum between mesons and baryons is only $15 \%$.

(iii) Using the meson cloud model, a method has been suggested to measure the pion structure function down to $x \approx 10^{-4}$, by exploiting the non-perturbative $\pi N$ and $\pi \Delta$ in Fock components of the nucleon which dominate the fragmentation of protons into fast neutrons and $\Delta$ 's.

From all that has been reviewed here it should be obvious that the meson cloud model represents an important link between classical nuclear physics and high-energy particle physics. In the first case mesons and baryons are the relevant degrees of freedom whereas the latter is dominated by quarks and gluons. The model which we have presented here extends the meson-baryon dynamics into the non-perturbative regime of QCD and represents therefore an important tool to help to explore it.

\section{ACKNOWLEDGMENTS}

It is a pleasure to thank the many colleagues who have contributed to our understanding of the issues presented here. We would like to thank particularly W.-P. Hwang, H. Holtmann, N. Isgur, J. T. Londergan, W. Melnitchouk, N.N. Nikolaev, A. W. Schreiber, A. I. Signal, S. Steffens, A. Szczurek and V. Zoller. One of us (J. S.) wish to thank Tony Thomas and Nathan Isgur for their hospitality during his visits at the University of Adelaide and Jefferson Laboratory where this work has 
been done. This work was supported in part by the Australian Research Council, the US Department of Energy under contract DE-AC05-84ER40150 and the Humboldt senior research award program.

\section{APPENDIX A: LAGRANGIANS}

Here we present the interaction Lagrangians we employ in our calculations. They are usually used in meson exchange models (MHE 87). $\phi$ denotes a spin-1/2 field $(N), \varphi$ a spin-3/2 field $(\Delta)$ of Rarita-Schwinger form; with $\pi$ pseudoscalar fields are denoted, with $\theta$ vector fields $(\rho, \omega)$ :

$L_{1}=g \cdot i \bar{\phi} \gamma_{5} \pi \phi$

$L_{2}=f \cdot \bar{\phi} \partial_{\mu} \phi \psi^{\mu}+$ h.c.

$L_{3}=g \cdot \bar{\phi} \gamma_{\mu} \theta^{\mu} \phi+f \cdot \bar{\phi} \sigma_{\mu \nu} \phi\left(\partial^{\mu} \theta^{\nu}-\partial^{\nu} \theta^{\mu}\right)$

$L_{4}=f \cdot i \bar{\phi} \gamma_{5} \gamma_{\mu} \psi_{\nu}\left(\partial^{\mu} \theta^{\nu}-\partial^{\nu} \theta^{\mu}\right)+h \cdot c$.

The anti-symmetric tensor $\sigma_{\mu \nu}$ here is defined as $\sigma_{\mu \nu}=\frac{i}{2}\left[\gamma_{\mu}, \gamma_{\nu}\right]$.

\section{APPENDIX B: VERTEX FUNCTIONS}

Here we list results ( $\mathrm{Hol}$ 95) for helicity dependent vertex functions $V_{I M R}^{2 \lambda^{\prime}}\left(y, k_{\perp}^{2}\right), y$ here denotes the longitudinal momentum fraction of the baryon in the nucleon; $\vec{k}_{\perp}=\left(k_{\perp} \cos \varphi, k_{\perp} \sin \varphi\right)$ the transverse momentum of the baryon with respect to the nucleon momentum. The contributions are listed according to particle helicities $\left(1 / 2 \rightarrow \lambda, \lambda^{\prime}\right)$, with $\lambda$ and $\lambda^{\prime}$ being the baryon and meson helicities respectively. 
a) Transitions for $L_{1}(N \pi, N \eta, \Sigma K, \Lambda K)$

$\frac{1}{2} \rightarrow+\frac{1}{2}, \quad 0 \quad \frac{g}{2} \frac{y m_{N}-m_{B}}{\sqrt{y m_{N}} m_{B}}$

$\frac{1}{2} \rightarrow-\frac{1}{2}, \quad 0 \quad \frac{g e^{-i \varphi}}{2} \frac{k_{\perp}}{\sqrt{y m_{N} m_{B}}}$

b) Transitions for $L_{2}\left(\Delta \pi, \Sigma^{*} K\right)$

$\frac{1}{2} \rightarrow+\frac{3}{2}, \quad 0 \quad-\frac{f e^{+i \varphi}}{2 \sqrt{2}} \frac{k_{\perp}\left(y m_{N}+m_{B}\right)}{y \sqrt{y m_{N} m_{B}}}$

$\frac{1}{2} \rightarrow+\frac{1}{2}, \quad 0 \quad \frac{f}{2 \sqrt{6}} \frac{\left(y m_{N}+m_{B}\right)^{2}\left(y m_{N}-m_{B}\right)+k_{1}^{2}\left(y m_{N}+2 m_{B}\right)}{y m_{B} \sqrt{y m_{N} m_{B}}}$

$\frac{1}{2} \rightarrow-\frac{1}{2}, \quad 0 \quad \frac{f e^{-i \varphi} k_{\perp}\left[\left(y m_{N}+m_{B}\right)^{2}-3 m_{B}\left(y m_{N}+m_{B}\right)+k_{\perp}^{2}\right]}{2 \sqrt{6}}$

$\frac{1}{2} \rightarrow \quad-\frac{3}{2}, \quad 0 \quad-\frac{f e^{-2 i \varphi}}{2 \sqrt{2}} \frac{k_{\perp}^{2}}{y \sqrt{y m_{N} m_{B}}}$

c) Transitions for $L_{3}\left(N \rho, N \omega, \Sigma K^{*}, \Lambda K^{*}\right)$

$$
\frac{1}{2} \rightarrow+\frac{1}{2}, \quad+1 \quad \frac{g e^{+i \varphi}}{\sqrt{2}} \frac{k_{\perp}}{(1-y) \sqrt{y m_{N} m_{B}}}-f \sqrt{2} e^{+i \varphi} \frac{k_{\perp} m_{N}}{\sqrt{y m_{N} m_{B}}}
$$

$\frac{1}{2} \rightarrow+\frac{1}{2}, \quad 0 \quad \frac{g}{2} \frac{k_{\perp}^{2}+m_{N} m_{B}(1-y)^{2}-y m_{M}^{2}}{(1-y) m_{M} \sqrt{y m_{N} m_{B}}}$

61 


$$
\begin{aligned}
& -\frac{f}{2} \frac{\left(y m_{N}-m_{B}\right)\left(y^{2} m_{N}^{2}-y\left(m_{N}^{2}+m_{B}^{2}+m_{M}^{2}\right)+m_{B}^{2}+k_{\perp}^{2}\right)}{y m_{M} \sqrt{y m_{N} m_{B}}} \\
& \frac{1}{2} \rightarrow \quad+\frac{1}{2}, \quad-1 \quad \frac{g e^{-i \varphi}}{\sqrt{2}} \frac{y k_{\perp}}{(1-y) \sqrt{y m_{N} m_{B}}}+f \sqrt{2} e^{-i \varphi} \frac{k_{\perp} m_{B}}{\sqrt{y m_{N} m_{B}}} \\
& \frac{1}{2} \rightarrow \quad-\frac{1}{2}, \quad+1 \quad-\frac{g}{\sqrt{2}} \frac{y m_{N}-m_{B}}{\sqrt{y m_{N} m_{B}}} \\
& -f \sqrt{2} \frac{k_{\perp}^{2}-\left(m_{N}+m_{B}\right)(1-y)\left(y m_{N}-m_{B}\right)}{(1-y) \sqrt{y m_{N} m_{B}}} \\
& \frac{1}{2} \rightarrow \quad-\frac{1}{2}, \quad 0 \quad-\frac{g e^{-i \varphi}}{2} \frac{k_{1}\left(m_{N}-m_{B}\right)}{m_{M} \sqrt{y m_{N} m_{B}}} \\
& -\frac{f e^{-i \varphi}}{2} \frac{k_{\perp}(1+y)\left(y^{2} m_{N}^{2}-y\left(m_{N}^{2}+m_{B}^{2}+m_{M}^{2}\right)+m_{B}^{2}+k_{\perp}^{2}\right)}{y(1-y) m_{M} \sqrt{y m_{N} m_{B}}} \\
& \frac{1}{2} \rightarrow \quad-\frac{1}{2}, \quad-1 \quad f \sqrt{2} e^{-2 i \varphi} \frac{k_{\perp}^{2}}{(1-y) \sqrt{y m_{N} m_{B}}}
\end{aligned}
$$

d) Transitions for $L_{4}\left(\Delta \rho, \Sigma^{*} K^{*}\right)$

$\frac{1}{2} \rightarrow \quad+\frac{3}{2}, \quad+1 \quad-\frac{f e^{+2 i \varphi}}{2} \frac{k_{\perp}^{2}}{y(1-y) \sqrt{y m_{N}} m_{B}}$

$\frac{1}{2} \rightarrow+\frac{3}{2}, \quad 0 \quad \frac{f e^{+i \varphi}}{\sqrt{2}} \frac{k_{\perp} m_{M}}{(1-y) \sqrt{y m_{N}} m_{B}}$

62 
$\frac{1}{2} \rightarrow \quad+\frac{3}{2}, \quad-1 \quad \frac{f}{2} \frac{m_{N} m_{B}(1-y)^{2}-y m_{M}^{2}}{(1-y) \sqrt{y m_{N}} m_{B}}$

$\frac{1}{2} \rightarrow+\frac{1}{2}, \quad+1 \quad \frac{f e^{+i \varphi}}{2 \sqrt{3}} \frac{k_{\perp}\left[k_{\perp}^{2}-2(1-y) m_{B}^{2}\right]}{y(1-y) m_{B} \sqrt{y m_{N}} m_{B}}$

$\frac{1}{2} \rightarrow \quad+\frac{1}{2}, \quad 0 \quad-\frac{f}{\sqrt{6}} \frac{m_{M}\left[k_{\perp}^{2}+m_{B}(1-y)\left(y m_{N}-m_{B}\right)\right]}{(1-y) m_{B} \sqrt{y m_{N}} m_{B}}$

$\frac{1}{2} \rightarrow \quad+\frac{1}{2}, \quad-1 \quad \frac{f e^{-i \varphi}}{2 \sqrt{3}} \frac{k_{\perp}\left[y m_{M}^{2}-2 m_{N} m_{B}(1-y)\right]}{(1-y) m_{B} \sqrt{y m_{N}} m_{B}}$

$\frac{1}{2} \rightarrow-\frac{1}{2}, \quad+1 \quad \frac{f}{2 \sqrt{3}} \frac{2(1-y) m_{B} k_{\perp}^{2}+m_{N} m_{M}^{2} y^{3}-(1-y)^{2} m_{B}^{3}}{y(1-y) m_{B} \sqrt{y m_{N}} m_{B}}$

$\frac{1}{2} \rightarrow-\frac{1}{2}, \quad 0 \quad \frac{f e^{-i \varphi}}{\sqrt{6}} \frac{k_{\perp} m_{M}\left(y m_{N}-(1-y) m_{B}\right)}{(1-y) m_{B} \sqrt{y m_{N}} m_{B}}$

$\frac{1}{2} \rightarrow-\frac{1}{2}, \quad-1 \quad \frac{f e^{-2 i \varphi}}{2 \sqrt{3}} \frac{k_{\perp}^{2} m_{N}}{(1-y) m_{B} \sqrt{y m_{N} m_{B}}}$

$\frac{1}{2} \rightarrow \quad-\frac{3}{2}, \quad+1 \quad \frac{f e^{-i \varphi}}{2} \frac{k_{\perp} m_{B}(1-y)}{y \sqrt{y m_{N} m_{B}}}$

$\frac{1}{2} \rightarrow-\frac{3}{2}, \quad 0 \quad 0$

$\frac{1}{2} \rightarrow \quad-\frac{3}{2}, \quad-1 \quad 0$

63 


\section{REFERENCES}

Abr+ 82 H. Abramowicz et al., (CDHS Collaboration), Z. Phys. C15, 19 (1982); C17, 1283 (1983).

Ada+ 94 D. Adams et al., Phys. Lett. B329, 399 (1994).

AEK 95 M. Alberg, J. Ellis, and D. Kharzeev, Phys. Lett. B356, 113 (1995).

Ald+ 90 D. M. Alde et al., Phys. Rev. Lett. 64, 2479 (1990).

Alt $82 \quad$ G. Altarelli, Phys. Rep. 81, 1 (1982).

Ama+ 91 P. Amandruz et al., Phys. Rev. Lett. 66, 2712 (1991); Phys. Lett. B292, 159 (1992).

Ant+ 93 P. L. Anthony et al., Phys. Rev. Lett. 71, 959 (1993).

Arn 94 M. Arneodo, Phys. Rep. 240, 301 (1994).

Arn+ 94 M. Arneodo et al., Phys. Rev. D50, R1 (1994).

Ash+89 J. Ashman et al., Nucl. Phys. B328(1989).

AT 80 J. L. Alonso and R. Tarrach (eds.), Quantum Chromodynamics, Springer, Berlin (1980).

AT 83 R. F. Alvarez-Estrada and A. W. Thomas, J. Phys. G9, 161 (1983).

Aub+ 83 J. Aubert et al., Phys. Lett. B123, 275 (1983).

Bad+ 96 B. Badelek et al., J. Phys. G22, 815 (1996).

Bal+ 94 A. Baldit et al., Phys. Lett. B332, 244 (1994).

Baz+95 A. O Bazarko et al., Z. Phys. C65, 189 (1995).

BCW 84 E. L. Berger, F. Coester, and R. B. Wiringa, Phys. Rev. D29, 398 (1984);

E. L. Berger and F. Coester, Annu. Rev. Nucl. Part. Sci. 37, 463 (1987).

Bha+ 84 S. Bhadra et al., DESY-preprint 94-084 (1994), Nucl. Inst. and Methods, A354, 479 (1995).

Bjo 66 J. D. Bjorken, Phys. Rev. 148, 1467 (1966).

Bjo 69 J. D. Bjorken, Phys. Rev. 179, 1547 (1969).

Blo+ 78 V. Blobel et al., Nucl. Phys. B135, 379 (1978).

Bod+ 73 A. Bodek et al., Phys. Rev. Lett. 30, 1087 (1973).

Bog 63 N. N. Bogoliubov, Ann. Inst. Henri Poincaré 8, 163 (1963).

BR 79 G. E. Brown and M. Rho, Phys. Lett. 82B, 177 (1979).

BR 79 G. E. Brown and M. Rho, Phys. Lett. B82, 177 (1979). 
Bro+ 95 S. J. Brodsky et al., Nucl. Phys. B441, 197 (1995).

BT 94 S. D. Bass and A.W. Thomas, Prog. Part. Nucl. Phys. 33, 449 (1994).

Clo 79 F. E. Close, An Introduction to Quarks and Partons, Academic Press, N.Y. (1979).

Cra+ 83 N. S. Craigie et al., Phys. Rep. 99, 69 (1983).

Das+ 88 S. Dasu et al., Phys. Rev. Lett 61, 1061 (1988).

Dei+ 91 S. Deister, M. F. Gari, W. Krumpelmann, and M. Mahlke, Few Body Systems 10, 1 (1991).

DLY 70 S. D. Drell, D. J. Levy, and T. M. Yan, Phys. Rev. D1, 1035 (1970).

DO 84 D. W. Duke and J. T. Owens, Phys. Rev. D30, 49 (1984).

DT 92 V. Dmitrasinovic and R. Tegen, Phys. Rev. C46, 1108 (1992).

DY $71 \quad$ S. D. Drell and T. M. Yan, Ann. Phys. 66, 578 (1971).

EJ 74 J. Ellis and R. L. Jaffe, Phys. Rev. D9, 1444 (1974).

EK 95 J. Ellis and M. Karliner, Phys. Lett. B341, 397 (1995).

ET 84 M. Ericson and A. W. Thomas, Phys. Lett. B148, 181 (1984).

Fey 72 R. P. Feynman, Photon Hadron Interactions, Benjamin, Reading, 1972.

FM 76 W. Flauger and F. Monning, Nucl. Phys. B109, 347 (1976).

FMS 89 L. L. Frankfurt, L. Mankiewicz and M. I. Strikman, Z. Phys. A334, 343 (1989).

Folit 90 C. Foudas et al., Phys. Rev. Lett. 64, 1207 (1990).

Fri 89 H. Fritzsch, Phys. Lett. B229, 291 (1989).

Gar+ 92 G. T. Garvey et al., FNAL proposal, P866 (1992).

GST 95 D. F. Geesaman, K. Saito, and A. W. Thomas, Annu. Rev. Nucl. Part. Sci. 45, 337 (1995).

Güt+ 84 F. Güttner, G. Chanfray, H. J. Pirner, and B. Povh, Nucl. Phys. A429, 389 (1984).

Hai+ 92 J. Haidenbauer, K. Holinde, V. Mull, and J. Speth, Phys. Lett. B291, 223 (1992).

HER 96 J. Phys. G22, Nr. 6 (1996), Ed. R. Devenish and J. Sterlin.

HHT 94 J. Haidenbauer, K. Holinde, and A. W. Thomas, Phys. Rev. C49, 2331 (1994).

HI 82 C. Hayne and N. Isgur, Phys. Rev. D25, 1944 (1982). 
HM 90 E. M. Henley and G. A. Miller, Phys. Lett. B251, 453 (1990).

Hol 93 H. Holtmann, Diplomarbeit, IKP-Forschungszentrum Jülich (1993), unpublished.

Hol 95 H. Holtmann, Dissertation, University of Bonn, Dez. 1995.

Hol+94 H. Holtmann, G. Levman, N. N. Nikolaev, A. Szczurek, and J. Speth, Phys. Lett. B338, 363 (1994).

Hol+ 96 H. Holtmann, N.N. Nikolaev, J. Speth, and A. Szczurek, Z. Phys. A353, 411 (1996).

HSB 91 W.-Y. P. Hwang, J. Speth, and G. E. Brown, Z. Phys. A339, 383 (1991).

HSS 93 H. Holtmann, A. Szczurek, and J. Speth, Jülich preprint KFA-IKP (TH)1993-33.

HSS 96 H. Holtmann, A. Szczurek, and J. Speth, Nucl. Phys. A596, 631 (1996).

Ito+ 81 A. S. Ito et al., Phys. Rev. D23, 604 (1981).

Jan 95 G. Janssen, Dissertation Mai 1995, University of Bonn.

Jan+93 G. Janssen, J. W. Durso, K. Holinde, B. C. Pearce, and J. Speth, Phys. Rev. Lett. 71, 1978 (1993).

JHS 94 G. Janssen, K. Holinde, and J. Speth, Phys. Rev. Lett. 73, 1332 (1994).

JT $95 \quad X$. Ji and J. Tang, Phys. Lett. B362, 182 (1995).

KFS 96 W. Köpf, L. L. Frankfurt, and M. Strikman, Phys. Rev. D53, 2586 (1996).

KL 91 S. Kumano and J. T. Londergan, Phys. Rev. D44, 717 (1991).

KP 95 B. Kopeliovich and B. Povh, preprint MPI-Heidelberg, MPIH-V12-1995.

Kub+ 85 K. Kubodera, Y. Kohymma, K. Oikawa and C. W. Kim, Nucl. Phys. A439, 695 (1985).

Kum 91 S. Kumano, Phys. Rev. D43, 59 (1991).

Leu+ 93 W.C. Leung, et al., Phys. Lett. B317, 655 (1993).

LeY+75 A. Le Yaounanc et al., Phys. Rev. D11, 2636 (1975).

Lle 83 C. H. Llewellyn Smith, Phys. Lett. B128, 107 (1983).

Lon+94 J. T. Londergan, G. T. Garvey, G. Q. Liu, E. N. Rodionov, and A. W. Thomas, Phys. Lett. B340, 115 (1994).

Lon+95 J. T. Londergan, G.Q. Liu, E. N. Rodionov and A.W. Thomas, Phys. Lett. B361, 110 (1995).

LP 82 E. Leader and E. Predazzi, An Introduction to Gauge Theories and the New Physics, Cambridge (1982). 
McG+ 92 P. L. McGaughey et al., Phys. Rev. Lett. 69, 1726 (1992).

MHE 87 R. Machleidt, K. Holinde, and Ch. Elster, Phys. Rep. 149, 1 (1987).

Mis+92 S.R. Mishra et al., Phys. Rev. Lett. 68, 3499 (1992).

MSR 93 A. D. Martin, W. J. Sterling, and R. G. Robert, Phys. Lett. B306, 145 (1993).

MSR 94 A. D. Martin, W. J. Sterling, and R. G. Roberts, Phys. Rev. D50, 6734 (1994).

MST 94 W. Melnitchouk, A. W. Schreiber, and A. W. Thomas, Phys. Rev. D49, 1183 (1994).

MT 93a W. Melnitchouk and A. W. Thomas, Phys. Rev. D47, 3783 (1993).

MT 93b W. Melnitchouk and A. W. Thomas, Phys. Rev. D47, 3794 (1993).

MT 95 W. Melnitchouk and A. W. Thomas, Z. Phys. A353, 311 (1995).

MT 96 W. Melnitchouk and A.W. Thomas, Acta Physica Polonica B27, 1407 (1996); Phys. Lett. B377, 11 (1996).

MTS 91 W. Melnitchouk, A. W. Thomas, and A.I. Signal, Z. Phys. A340, 85 (1991).

Mut 87 T. Muta, Foundations of Quantum Chromodynamics, World Scientific, Singapore (1987).

NRS 78 M. M. Nagels, T. A. Rijken, and J. J. de Swart, Phys. Rev. D17, 768 (1978).

Owe 91 J. F. Owens, Phys. Lett. B266, 126 (1991).

PDG 92 Particle Data Group, Phys. Rev. D45,1 (1992).

PP 76 G. Parisi and G. Petronzio, Phys. Lett. B62, 331 (1976).

PP 92 H. J. Pirner and B. Povh, in Proceedings of the Italian Physical Society, Vol. 44, Ed. J. Arvieux and E. De Santis (1992).

RTL 94 E. N. Rodionov, A. W. Thomas, and J. T. Londergan, Mod. Phys. Lett. A9, 1799 (1994).

Sac 83 C. T. Sachrajda, in Gauge Theories in High Energy Physics, North Holland, Amsterdam (1983).

Sat 92 E. Sather, Phys. Lett. B274 (1992).

SHT 95 F. M. Steffens, H. Holtmann, and A.W. Thomas, Phys. Lett. B358, 139 (1995).

SMM 92 A. W. Schreiber, P. J. Mulders, and H. Meyer, Phys. Rev. C46, 458 (1992). 
SS 93 A. Szczurek and J. Speth, Nucl. Phys. A555, 249 (1993).

SSG 94 A. Szczurek, J. Speth, and G. T. Garvey, Nucl. Phys. A570, 765 (1994).

SST 91 A. W. Schreiber, A. I. Signal, and A. W. Thomas, Phys. Rev. D44, 2653 (1991).

ST 87 A. I. Signal and A. W. Thomas, Phys. Lett. B191, 205 (1987).

ST 88 A. W. Schreiber and A. W. Thomas, Phys. Lett. B215, 141 (1988).

ST 89 A. I. Signal and A. W. Thomas, Phys. Rev. D40, 2832 (1989).

ST 94 F. M. Steffens and A.W. Thomas, Adelaide -preprint ADP-94-27/T166.

ST 96 F. M. Steffens and A. W. Thomas, Adelaide-preprint, ADP-96-12/T215.

STL 90 A. W. Schreiber, A. W. Thomas and J. T. Londergan, Phys. Rev. D42, 2226 (1990).

Sul 72 J. D. Sullivan, Phys. Rev. D5, 1732 (1972).

Sut+ 92 P. J. Sutton, A. D. Martin, R. G. Roberts, and W. J. Stirling, Phys. Rev. D45, 2349 (1992).

SV 90 G. M. Shore and G. Veneziano, Phys. Lett. B224, 75 (1990).

SZ 95 J. Speth and V. Zoller, Phys. Lett. B351, 533 (1995).

Szc+ 96 A. Szczurek, M. Ericson, H. Holtmann, and J. Speth, Nucl. Phys. A596 397 (1996).

TH 90 A. W. Thomas and K. Holinde, Phys. Rev. C42, R1195 (1990).

Tho 83 A. W. Thomas, Phys. Lett. B126, 97 (1983).

Tho 84a A. W. Thomas, in Progress in Nuclear and Particle Physics 11, 325 (1984); 20, 21 (1988).

Tho 84b A. W. Thomas, Adv. Nucl. Phys. 13, 1 (1984).

TM 93 A. W. Thomas and W. Melnitchouk in: Proceedings of the JSPS-INS Spring, School (Shimoda, Japan); World Scientific, Singapore 1993.

TMT 82 S. Theberge, G. A. Miller, and A. W. Thomas, Can. J. Phys. 60, 59 (1982).

Wei 66 S. Weinberg, Phys. Rev. 150, 1313 (1966).

Ynd 83 F. Yndurain, Quantum Chromodynamics, Springer, Berlin (1983).

ZEU 93 ZEUS-Collaboration, The ZEUS-detector, status report, 1993.

Zol 92 V. R. Zoller, Z. Phys. C53, 443 (1992).

Zol 93 V. R. Zoller, Z. Phys. C60, 141 (1993); Mod. Phys. Lett. A8, 1113 (1993). 


\section{TABLE 4.1}

Largest meson admixtures in the nucleon [in \%]

bare

$N-\pi$

$N-\rho$

$\Delta-\pi$

$\Delta-\rho$

58

18

13

6

2

TABLE 4.2

Comparison of measured ratios of quarkmomenta (CCFR-data (Fou+90)) with theoretical results obtained with MCM (Hol 95).

$\operatorname{Exp}$

Fit I

Fit II

$\mathrm{K}=\frac{2\langle x s\rangle}{\langle x \bar{u}\rangle+\langle x \bar{d}\rangle}$

$0.44_{-0.07-0.02}^{+0.09+0.07}$

0.46

0.54

$\eta_{s}=\frac{2\langle x s\rangle}{\langle x u\rangle+\langle x d\rangle}$

$0.057_{-0.008-0.002}^{+0.010+0.007}$

0.069

0.081

$R_{Q}=\frac{\langle x \bar{u}\rangle+\langle x \bar{d}\rangle+\langle x \bar{s}\rangle}{\langle x u\rangle+\langle x d\rangle+\langle x s\rangle}$

$0.153 \pm 0.034$

0.176

0.182

TABLE 4.3

Gottfried sum-rule with different meson contributions $\left(S_{G}^{\exp }=\mathbf{0 . 2 3 5} \pm 0.026\right)$. In each line the contribution of the given Fock-states are added (HSS 96).

$\langle\bar{u}-\bar{d}\rangle$

$S_{G}$

bare nucleon

1

0

0.333

$+\pi N$

0.755

$-0.141$

0.239

$+\pi \Delta$

0.697

$-0.106$

0.263

all

0.580

$-0.142$

0.238 
TABLE 4.4

A list of all possible semileptonic decays of baryons within the nucleon octet. The axial couplings $g_{A}$ has been calculated at the tree-level in the SU(6) model $(F=2 / 3$ and $D=1)$ and in the SU(3) model $F$ and $D(F=0.44, D=0.82)$ fitted. Moreover, the results of the same models are shown with inclusion of the meson cloud. For the $S U(3)$ case we find $F=0.53$ and $D=1.15$.

\begin{tabular}{|c|c|c|c|c|c|c|c|}
\hline & Decay & & $S U(6)$ & $S U(3)$ & $\begin{array}{c}M C \\
S U(6)\end{array}$ & $\begin{array}{c}M C, \\
S U(3)\end{array}$ & $g_{A} \exp$ \\
\hline$n$ & $\rightarrow$ & $p$ & 1.67 & 1.257 & 1.241 & 1.257 & $1.2573 \pm 0.0028$ \\
\hline$\Sigma^{+}$ & $\rightarrow$ & $\hat{\Lambda}$ & 0.82 & 0.67 & 0.66 & 0.74 & \\
\hline$\Sigma$ & $\rightarrow$ & $\Sigma^{0}$ & 0.94 & 0.62 & 0.77 & 0.64 & \\
\hline$\Sigma$ & $\rightarrow$ & $\Lambda$ & 0.82 & 0.67 & 0.65 & 0.75 & $0.60 \pm 0.03$ \\
\hline$\Sigma^{+}$ & $\rightarrow$ & $\Sigma^{0}$ & -0.94 & -0.62 & -0.77 & -0.64 & \\
\hline$\Xi$ & $\rightarrow$ & $\Xi^{0}$ & 0.33 & 0.38 & 0.27 & 0.49 & \\
\hline$\Lambda$ & $\rightarrow$ & $\rho$ & & -0.87 & -0.96 & -0.89 & $-0.857 \pm 0.018$ \\
\hline$\Sigma^{0}$ & $\rightarrow$ & $\rho$ & 0.24 & 0.27 & 0.19 & 0.31 & \\
\hline$\Sigma$ & $\rightarrow$ & $n$ & 0.33 & 0.38 & 0.27 & 0.49 & $0.34 \pm 0.05$ \\
\hline$\Xi^{0}$ & $\rightarrow$ & $\Sigma^{+}$ & 1.67 & 1.26 & 1.37 & 1.39 & \\
\hline$\Xi^{-}$ & $\rightarrow$ & $\Lambda$ & 0.41 & 0.20 & 0.35 & 0.16 & $0.31 \pm 0.06$ \\
\hline & $\rightarrow$ & $\Sigma^{0}$ & 1.18 & 0.89 & 0.97 & 0.98 & \\
\hline & $x^{2} / N$ & & 4369 & 2.0 & 8.5 & 6.5 & \\
\hline
\end{tabular}


TABLE 4.5

The Ellis-Jaffe sum rule for proton and neutron, the Bjorken sum rule and the axial flavour singlet coupling constant obtained with inclusion of different Fock states: octet baryons with pseudoscalar mesons (oct, ps), octed and decuplet baryons with pseudoscalar mesons (oct, decu, ps) and with vector mesons in addition (all). The axial coupling constants for the SU(3) case are: $F=0.53, D=1.15$ for (oct, ps) and $F=0.48, D=0.91$ for (oct, decu, ps) and $F=0.53, D=1.15$ (all).

\begin{tabular}{rccccc}
\hline & $S_{E s}^{p}$ & $S_{E J}^{n}$ & $S_{B}$ & $\Delta q_{0}$ & $\Delta s_{o}$ \\
& & & & & \\
\hline SU(6) tree & 0.278 & 0 & 0.278 & 1 & 0 \\
oct, ps & 0.212 & 0.004 & 0.208 & 0.779 & 0.004 \\
oct, decu, ps & 0.233 & -0.010 & 0.243 & 0.804 & 0.002 \\
all & 0.220 & 0.011 & 0.209 & 0.846 & 0.017 \\
& & & & & \\
\hline SU(3) tree & 0.173 & -0.037 & 0.210 & 0.489 & 0 \\
oct, ps & 0.154 & -0.056 & 0.210 & 0.356 & 0.003 \\
oct, decu, ps & 0.169 & -0.041 & 0.210 & 0.461 & 0.001 \\
all & 0.179 & -0.031 & 0.210 & 0.541 & 0.018 \\
$\therefore \quad$ & & & & & \\
\hline
\end{tabular}




\section{FIGURE-CAPTIONS}

Fig: 2.1: Summary of structure function data taken with neutrinos and muons by various groups.

Fig. 3.1: DIS from the virtual (a) meson and (b) baryon components of a physical nucleon.

Fig. 3.2: Time-ordered diagrams moving (a) forwards and (b) backwards in time. Time is increasing from left to right.

Fig. 3.3: Various splitting functions obtained by means of TOPT in the infinite momentum frame ( $\mathrm{Hol} 95)$.

Fig. 3.4: Splitting functions $f_{n v}(y)$ evaluated using covariant and time-ordered perturbation theory. The covariant function is as in Fig. 3.5. The cut-offs in the form factors are chosen to give the same value for $\langle n\rangle_{m \mathrm{v}}=0.235$ (TM 93).

Fig. 3.5: Splitting functions $f_{\pi V}(y)$ and $f_{N \pi}(1-y)$ with dipole form factors and cutoffs chosen to give $\langle n\rangle_{\pi V}=\langle n\rangle_{N \pi}=0.235$ (TM 93).

Fig. 3.6: The splitting functions $f_{p \pi \%}(y)$ (solid), $\Delta f_{p \pi \%}(y)$ (dash-dotted), $f_{p \pi \%}^{+1 / 2}(y)$ (dashed) and $f_{p \pi \%}^{-1 / 2}(y)$ (dotted) with a cut-off parameter of $\Lambda=1.08 \mathrm{GeV}$, (HSS 93).

Fig. 4.1: One-Boson-Exchange diagrams for $n$ and $\Lambda$ production.

Fig. 4.2: Differential cross sections for $p p \rightarrow n X$ and $p p \rightarrow \Lambda X$ (FM 76, Blo+ 78). Shown are the OBE contributions: pseudoscalar meson (dashed), vector mesons (dotted) and their sum (solid) (HSS 96).

Fig. 4.3: Fit to the experimental data to determine the quark distributions of the 'bare' nucleon, at $Q^{2}=4 \mathrm{GeV}^{2}($ Arn +94 , Leu+ 93). The thick and thin dashed curves are the 'bare' distributions of fit I and fit II, respectively. The thick and thin full line represent the results of the corresponding meson-cloud model calculation ( $\mathrm{Hol} 95)$. The scale of the $\bar{q}(x)$ 
experimental data (Mis+92) is $Q^{2}=3 \mathrm{GeV}^{2}$ (circles) and $Q^{2}=5 \mathrm{GeV}^{2}$ (squares).

Fig. 4.4: Quark distribution functions of the bare (dashed) and the physical nucleon (Hol 95). Differences between fit I and fit II (thin and thick line) can hardly been seen.

Fig. 4.5: Total valence distribution (SHT 95) in the bag-model and in the bag dressed with mesons compared with the MRS parametrization (MSR 93) of the data. The quark distributions are evolved in next-to-leading order QCD.

Fig. 4.6: Effect of the Pauli exclusion principle on the proton-neutron structure function difference, as a function of X (TM 93). The dotted (without meson corrections) and solid (with meson corrections) curves are for $P=0$ (largest curves) $0.05,0.1$ and 0.15 (smallest curves).

Fig. 4.7: Different contributions to the asymmetry of the sea quark distributions in the model of ref. (HSS 96, Hol 95).

Fig. 4.8: Drell-Yan process: An antiquark (quark) from a beam proton (A) annihilates with a quark (antiquark) from the target proton (B) and create a lepton pair.

Fig: 4.9: Cross section for the production of the dilepton pairs in proton-deuteron collisions. Shown is the fit of the $K$-factor for various quark distributions to the experimental data $(\mathrm{Szc}+96)$.

Fig. 4.10: Drell-Yan ratio for iron/deuterium and tungsten/deuterium. The solid line is the result of $M C M$, the dashed line the asymmetric $\operatorname{MSR}(A)$ and the dash-dotted line the symmetric $\operatorname{MSR}\left(S_{0}^{\prime}\right)$ parametrization (Szc+ 96). Data are taken from ref. (Ald +90$)$.

Fig. 4.11: The slope of the rapidity distribution. The full and dashed result is calculated with asymmetric sea quark distributions, the dotted and dashdotted with symmetric ones (Szc+ 96).

Fig. 4.12: A two-dimensional map of the Drell-Yan asymmetry as a functions of $x_{1}$ and $x_{2}$ (Szc+ 96). Shown are results obtained with the Owens parametrization (Owe 91) (left-upper corner), symmetric $\operatorname{MSR}\left(S_{0}^{\prime}\right)$ (right- 
upper corner), new $M R S(A)$ with the $\bar{u}-\bar{d}$-asymmetry built in (left-lower corner) and prediction of the meson cloud model (HSS 96, Hol 95) (rightlower corner). Note the change of the sign in the lower panels.

Fig. 4.13: Drell-Yan asymmetry along the $x_{1}=x_{2}$ diagonal (Szc+96). The meaning of the lines here is the same as in Fig. 4.11. The data point is taken from the NA51 Collaboration at CERN (Bal+ 94).

Fig. 4.14: The $\bar{u}(x) / \bar{d}(x)$ ratio as obtained from the meson cloud model (Hol+ 96) and the $\operatorname{MSK}(A)$ parametrization (dashed line) compared with the experimental result of the NA51 Collaboration (Bal+ 94).

Fig. 4.15: Polarization asymmetry for the $\pi$-exchange (upper curves) and parton fragmentation (lower curve) models. The solid and dashed lines are for CEBAF and HERMES kinematics, respectively.

Fig. 4.16: Polarization asymmetry for the $K$-exchange (solid) model of $\Lambda$ production, compared with a leading fragmentation approximation estimate for the parton fragmentation process (dashed).

Fig. 4.17: Corrections to an axial current in the meson cloud model.

Fig. 5.1: The pion (rho) exchange contributions to the inclusive neutron production (a) in $p p$-scattering and (b) neutron and (c) $\Delta^{++}$production in deep-inelastic scattering. Diagram (d) shows the diffractive production of $N^{* \prime} s$ by pomeron exchange.

Fig. 5.2: Longitudinal momentum distribution of (a) neutrons, (b) protons and (c) $\Delta^{++}$. The contributions of the $\pi$ and $\rho$ exchange mechanisms are shown by the dashed and dotted line, respectively. The contribution from the pomeron exchange mechanism to the $p \rightarrow p$ fragmentation is shown by the dash-dotted line $(\mathrm{Hol}+94)$.

Fig. 5.3: Longitudinal momentum distribution of neutrons (see Fig. 2 a.) with the extra condition $p_{\perp}^{2}<0.1(\mathrm{GeV} / \mathrm{c})^{2} \quad$ (lower curves) compared to the unconstrained one (upper curves, see Fig. 2 a.). The $\pi$ exchange contributions are shown by dashed lines; the $\rho$ exchange contributions by the dotted lines. 


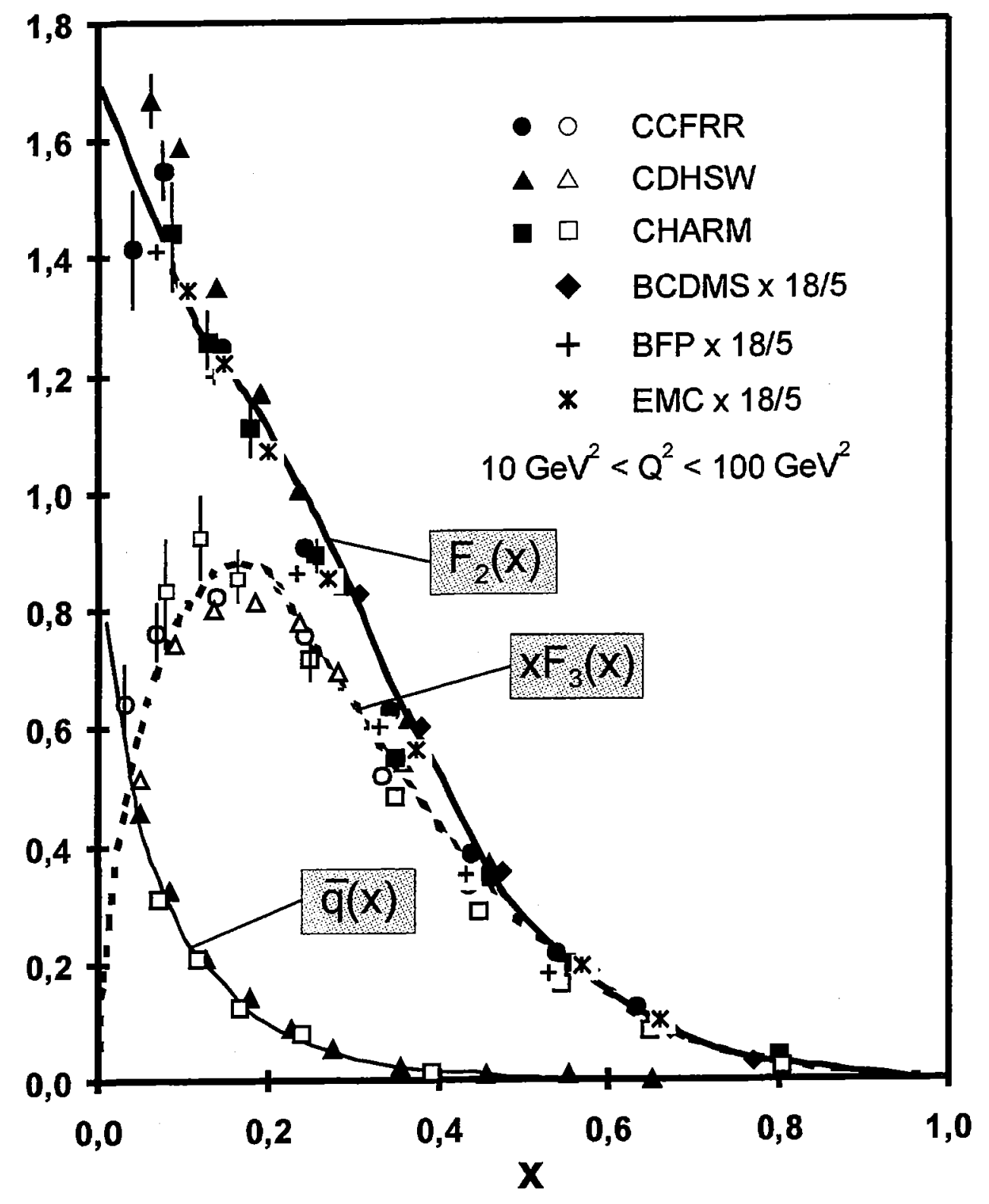

Fig. 2.1 

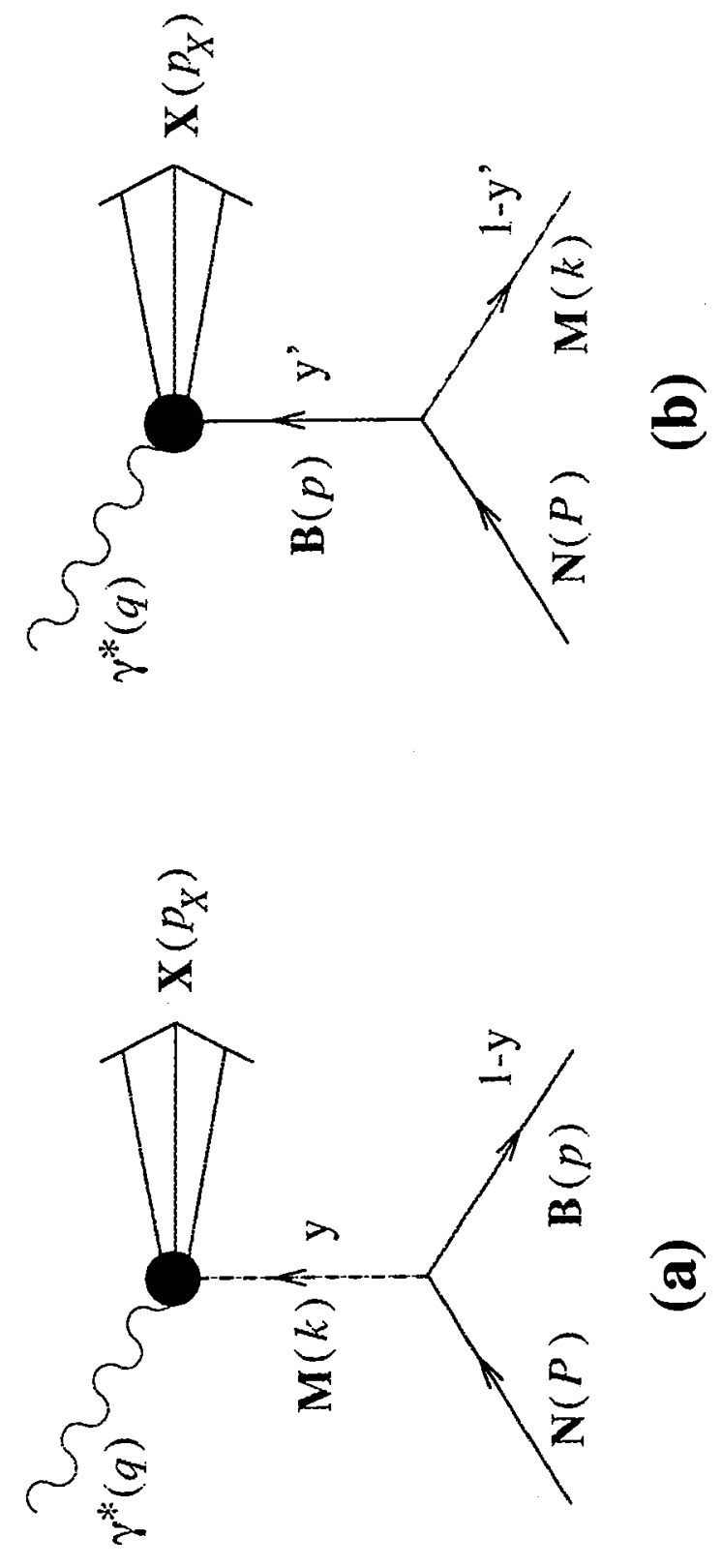

Fig. 3.1 


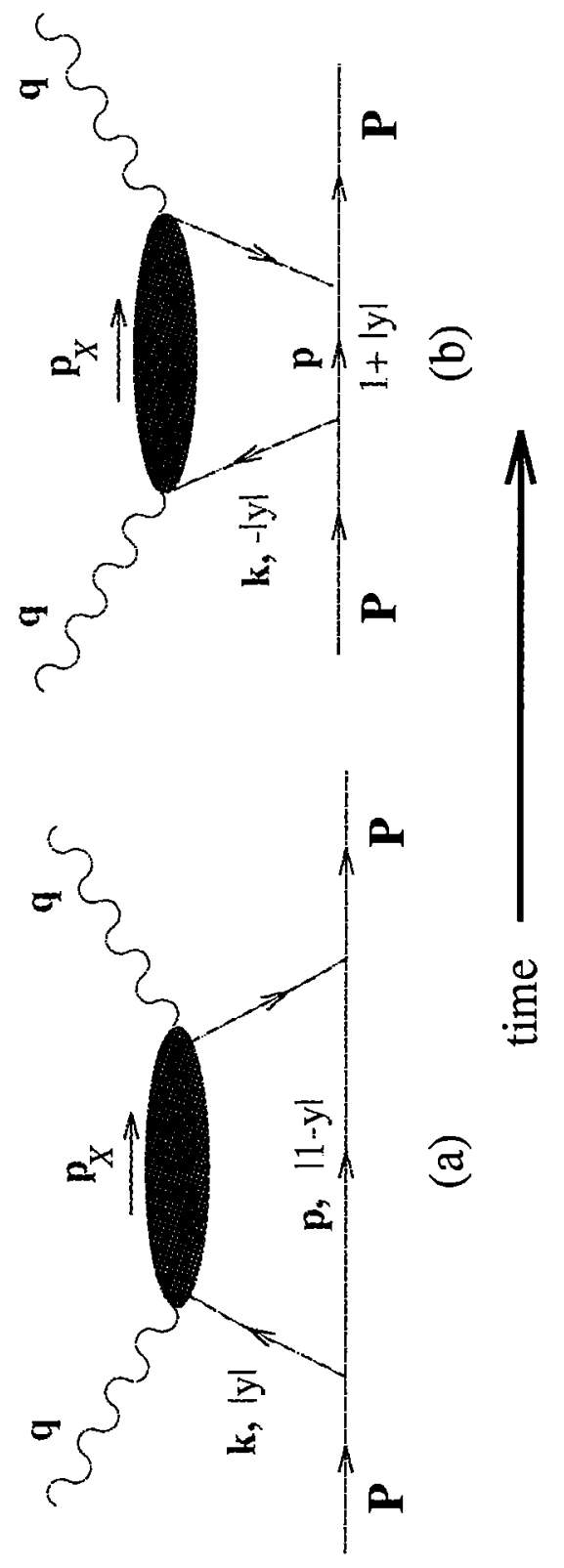

Fig. 3.2 


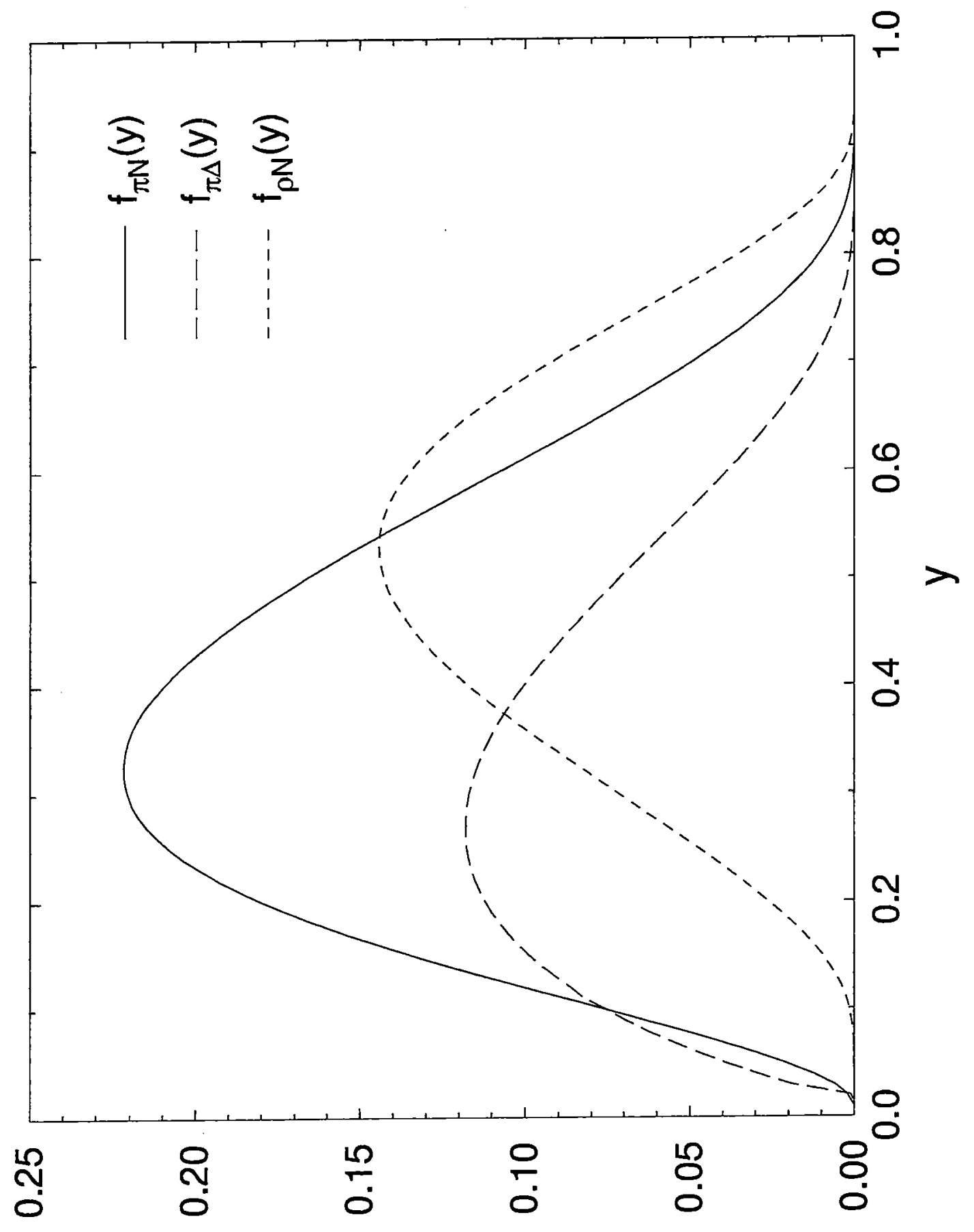

Fig. 3.3 




Fig. 3.4 


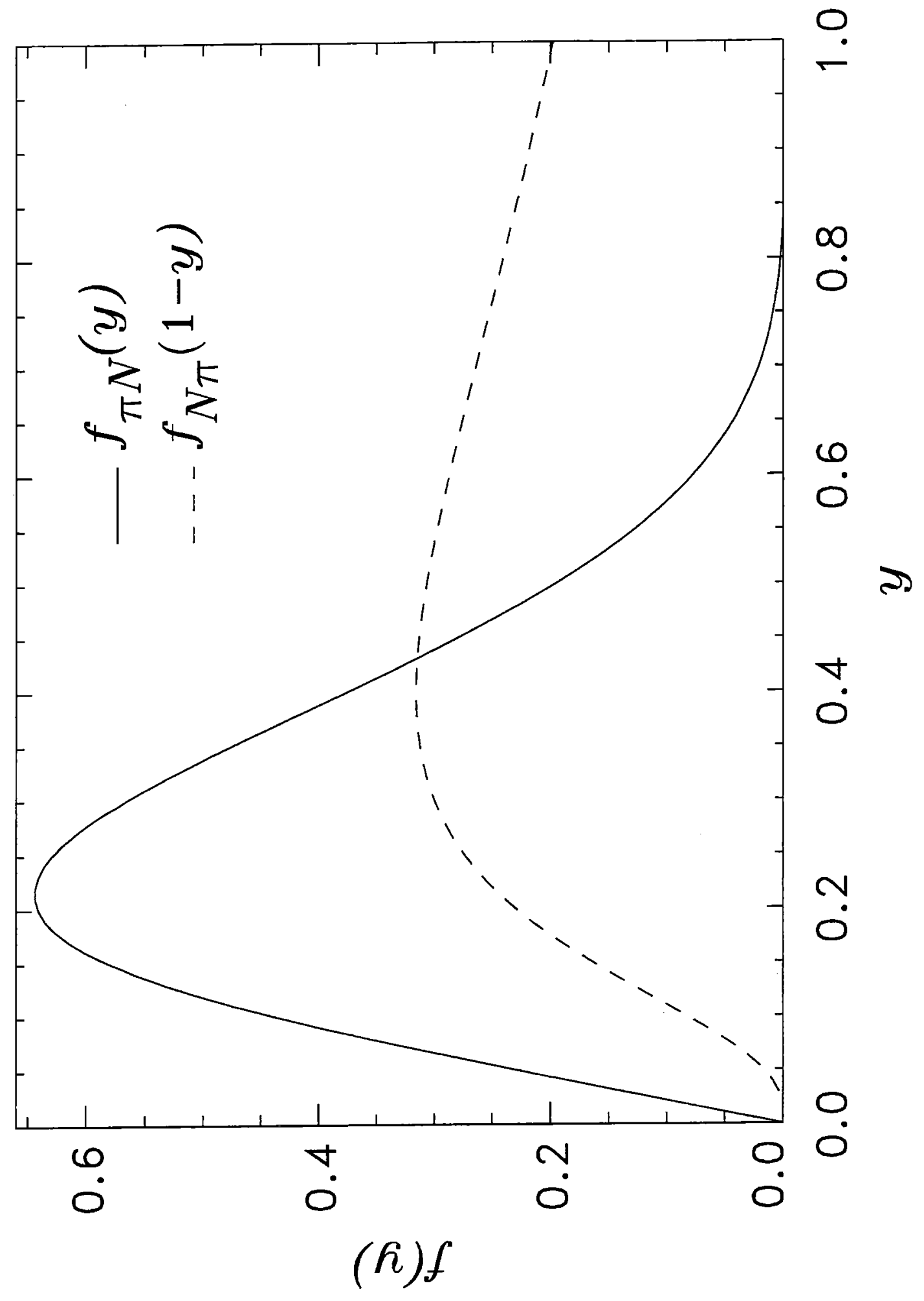

Fig. 3.5 


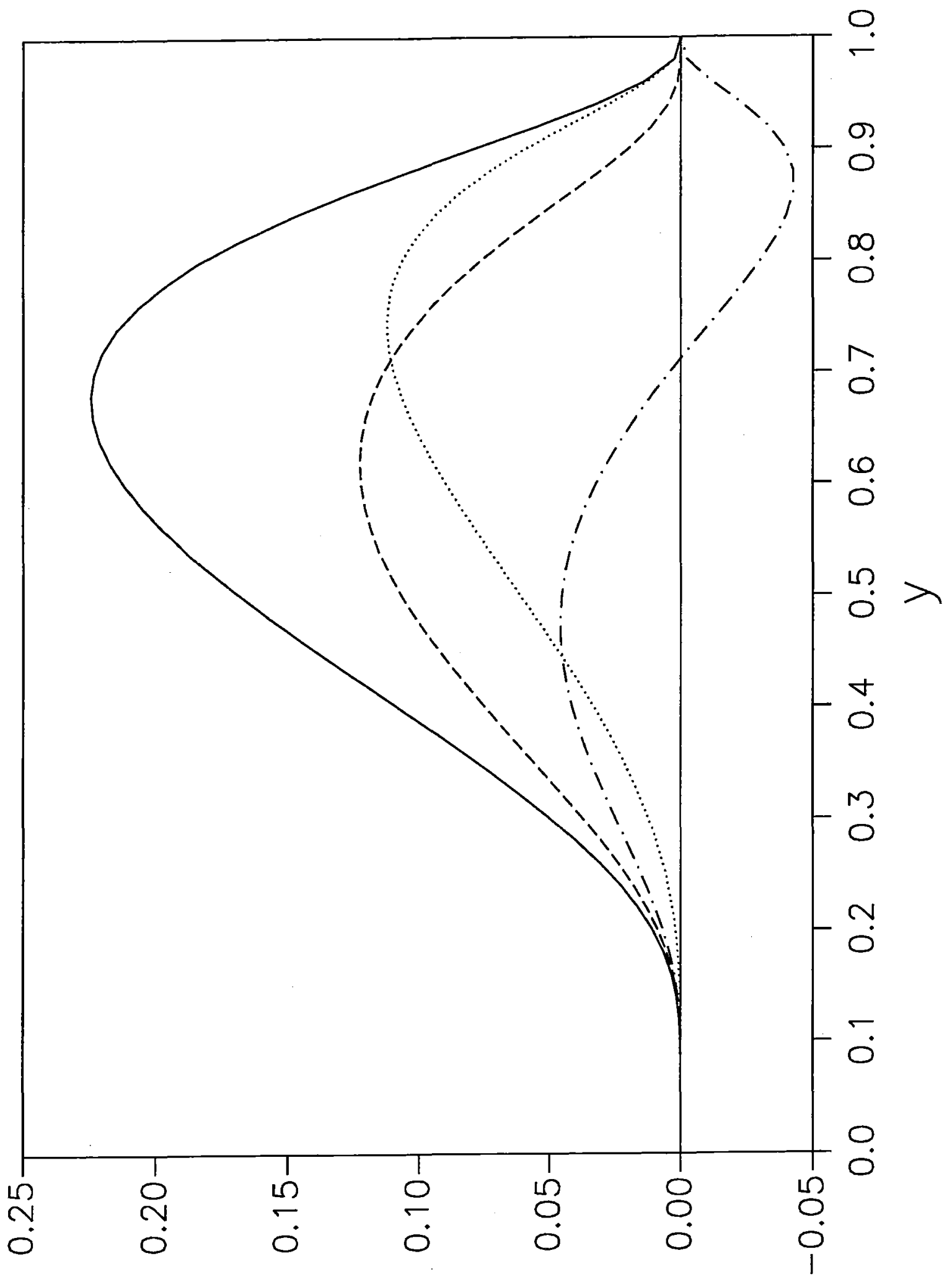

Fig. 3.6 

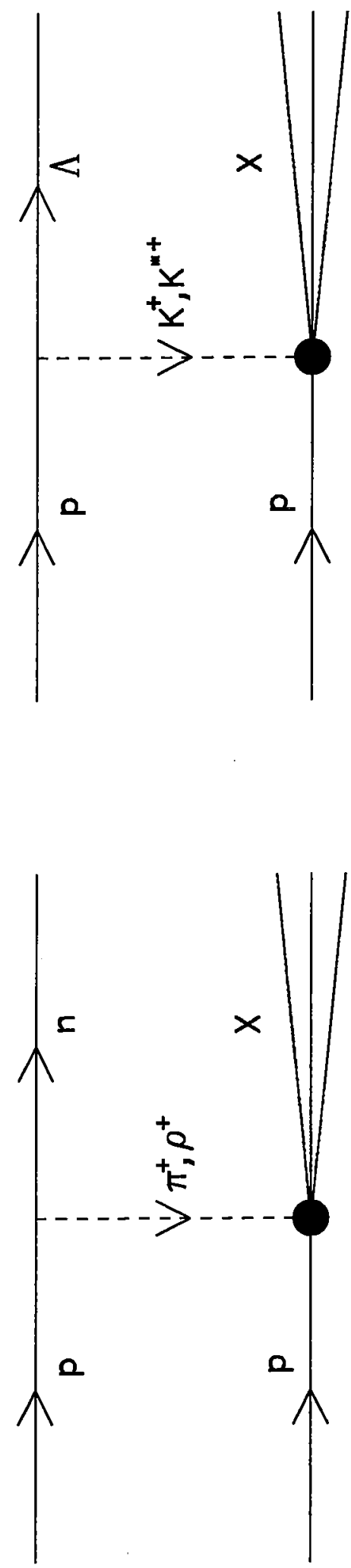

Fig. 4.1 

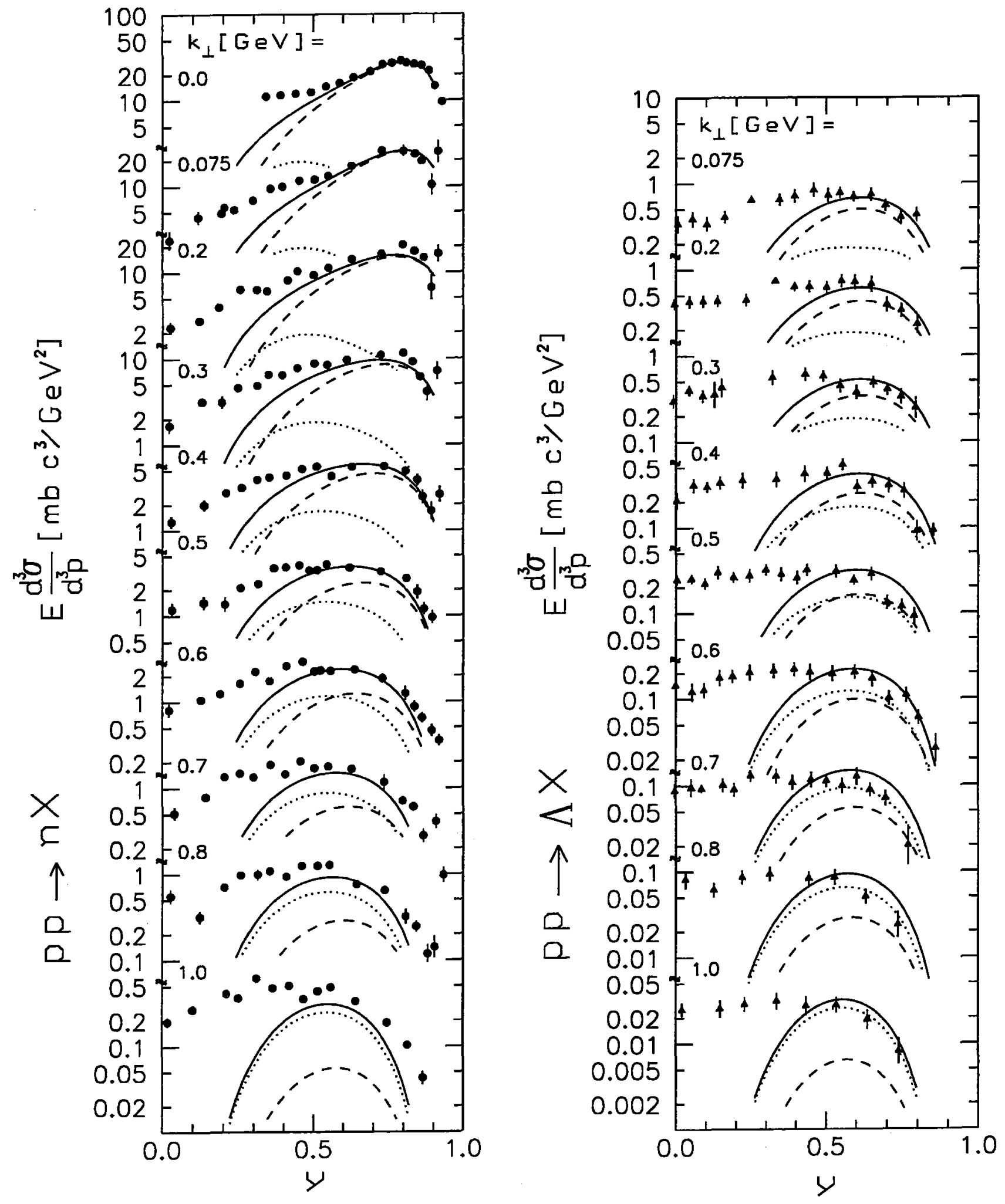

Fig. 4.2 

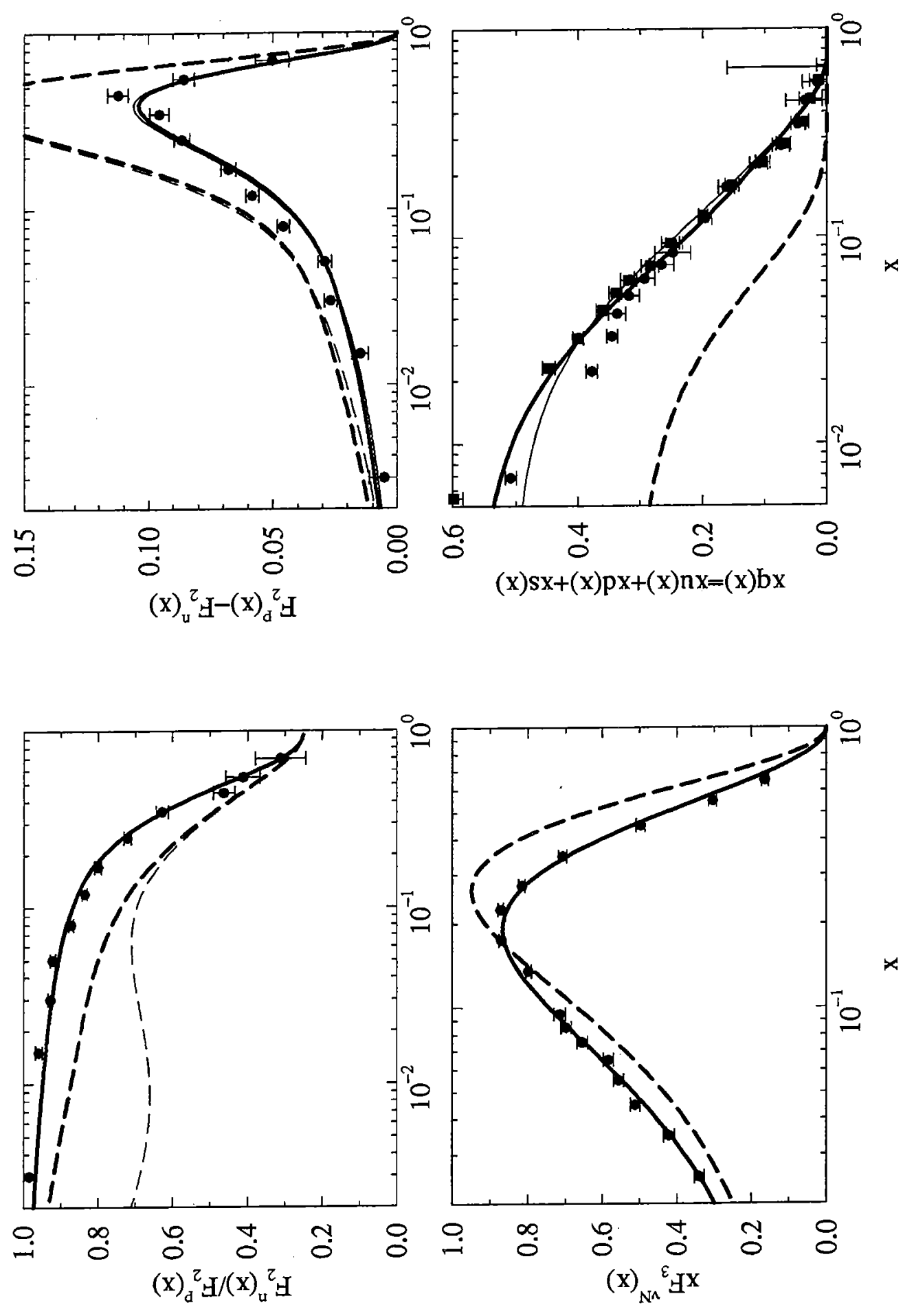

Fig. 4.3 


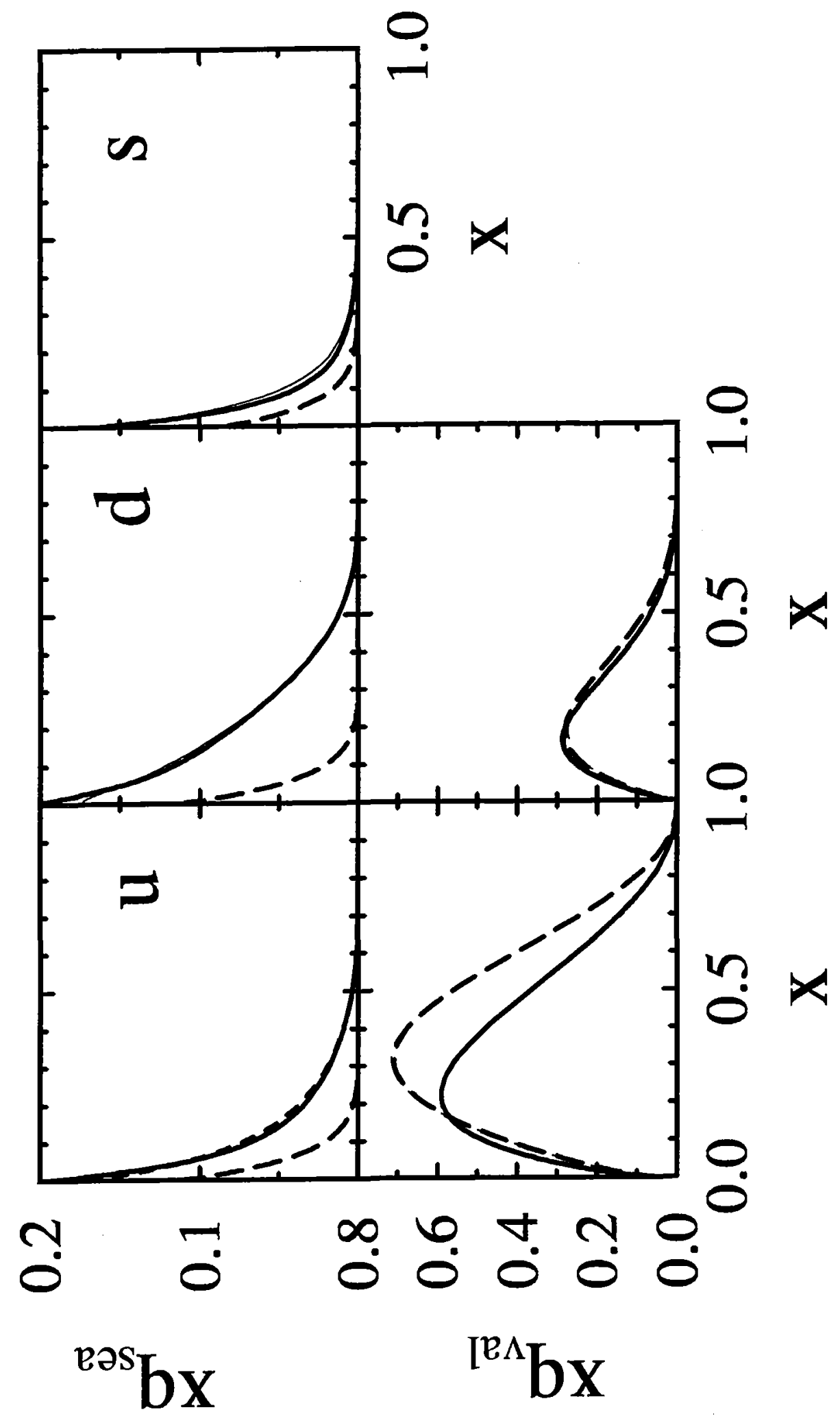

Fig. 4.4

85

9 


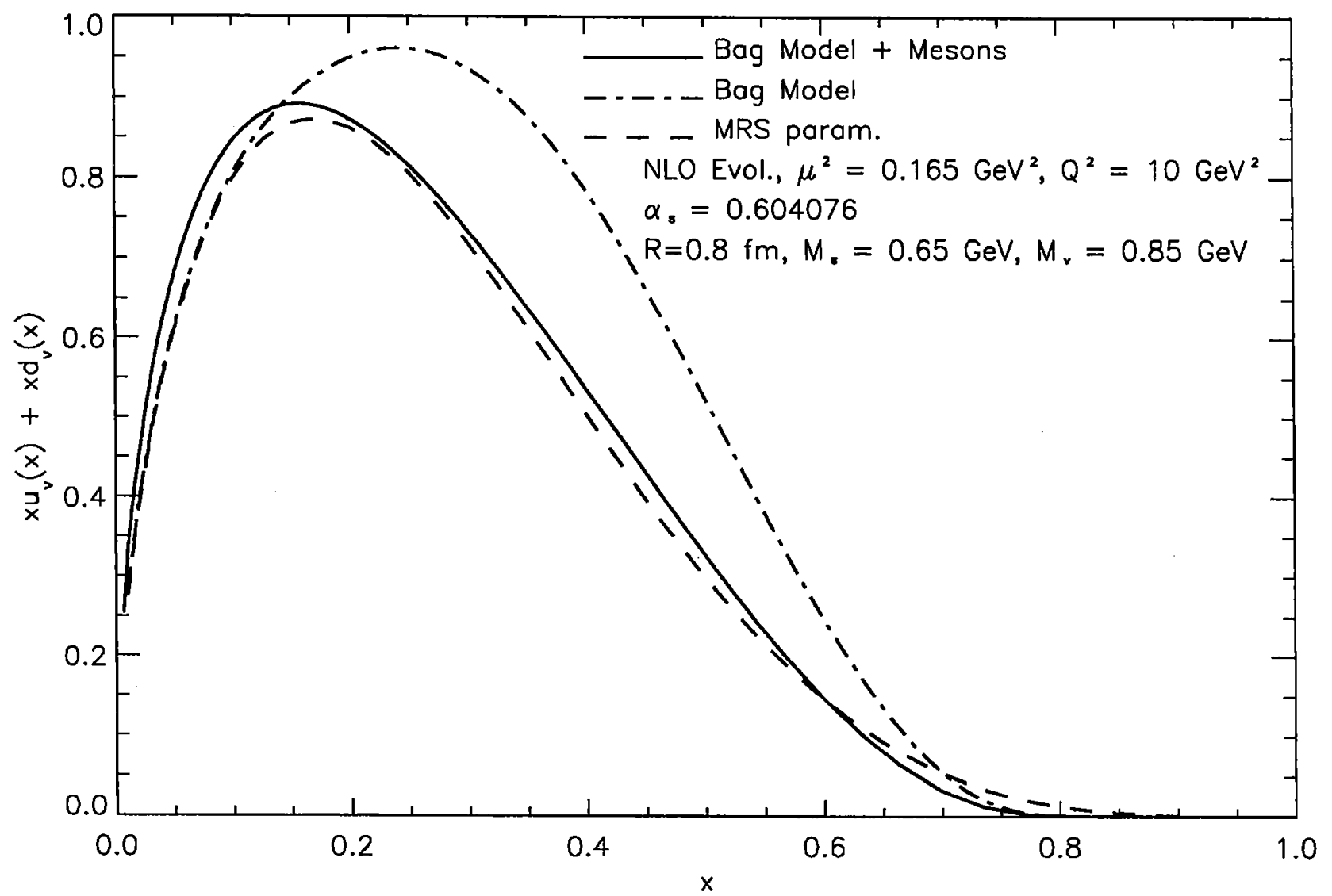




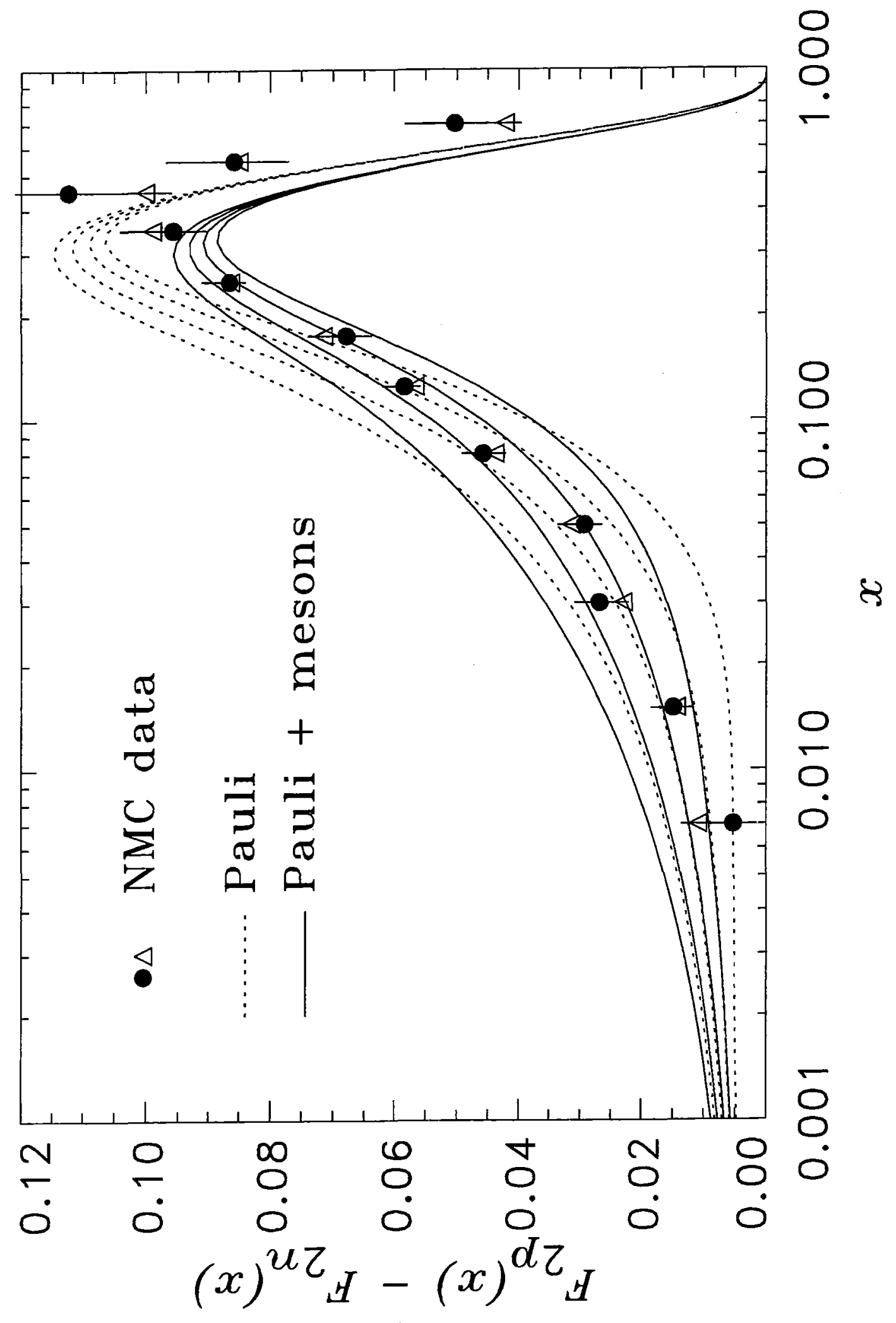

Fig. 4.6 


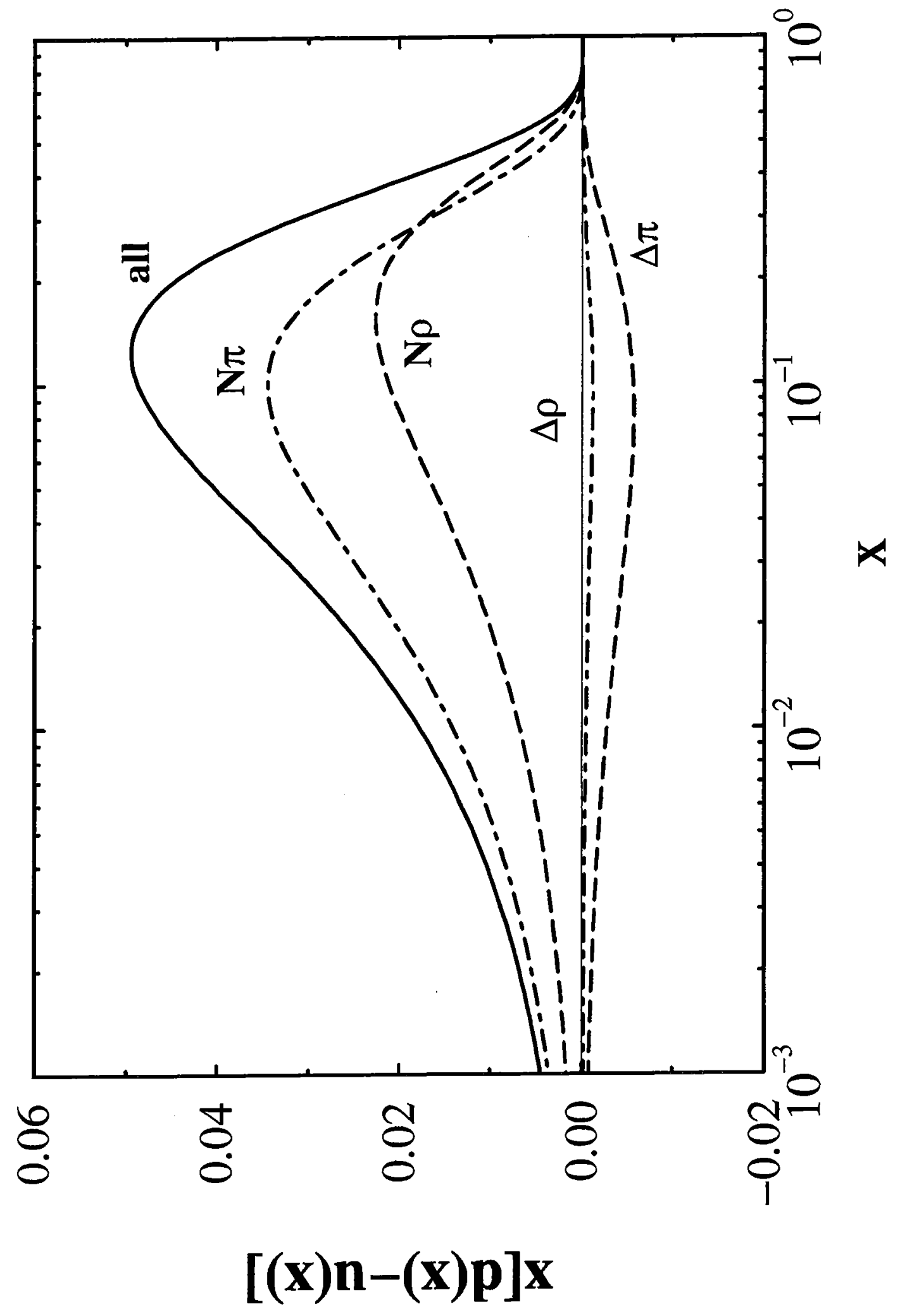

Fig. 4.7 


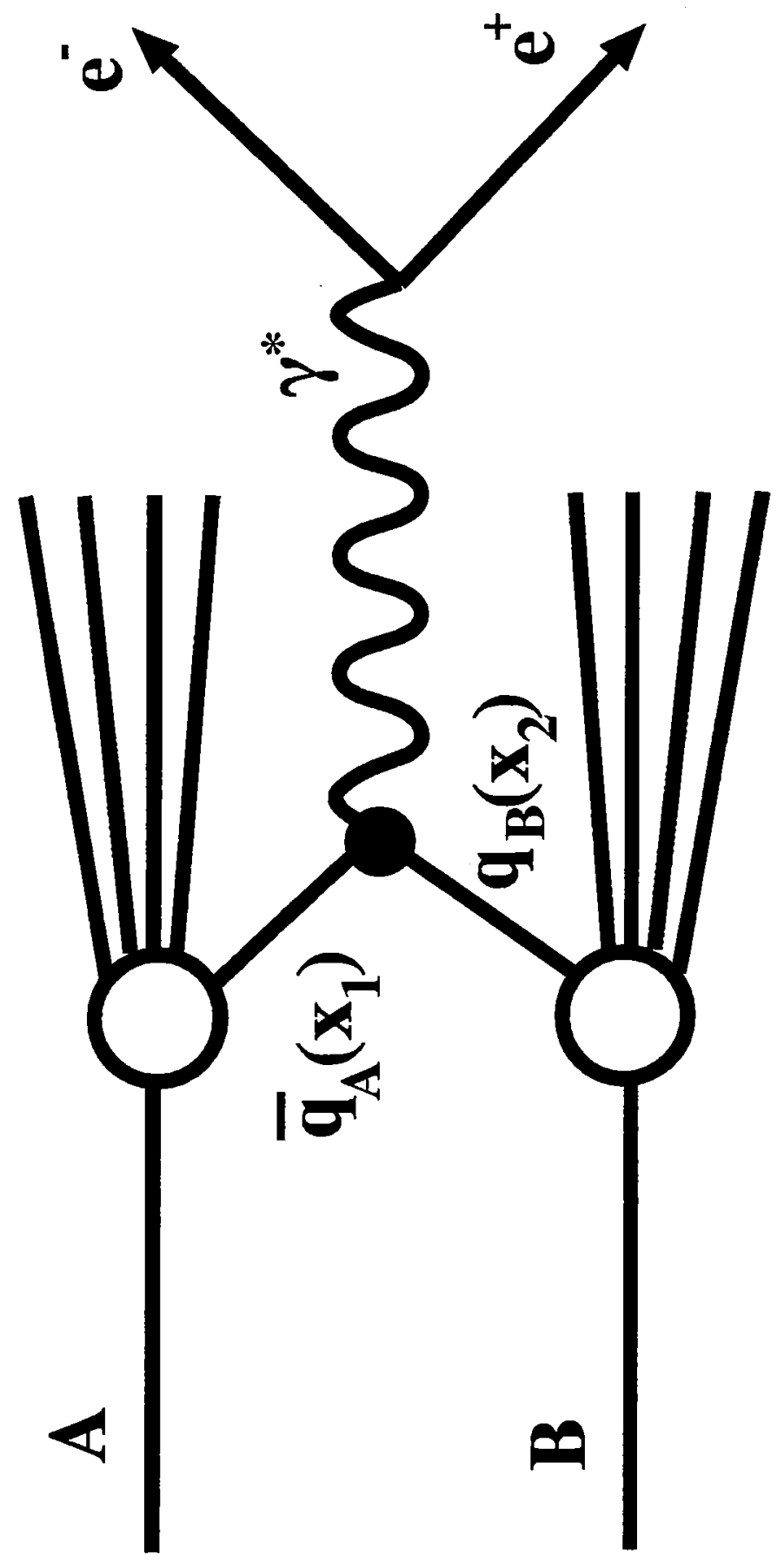

Fig. 4.8 


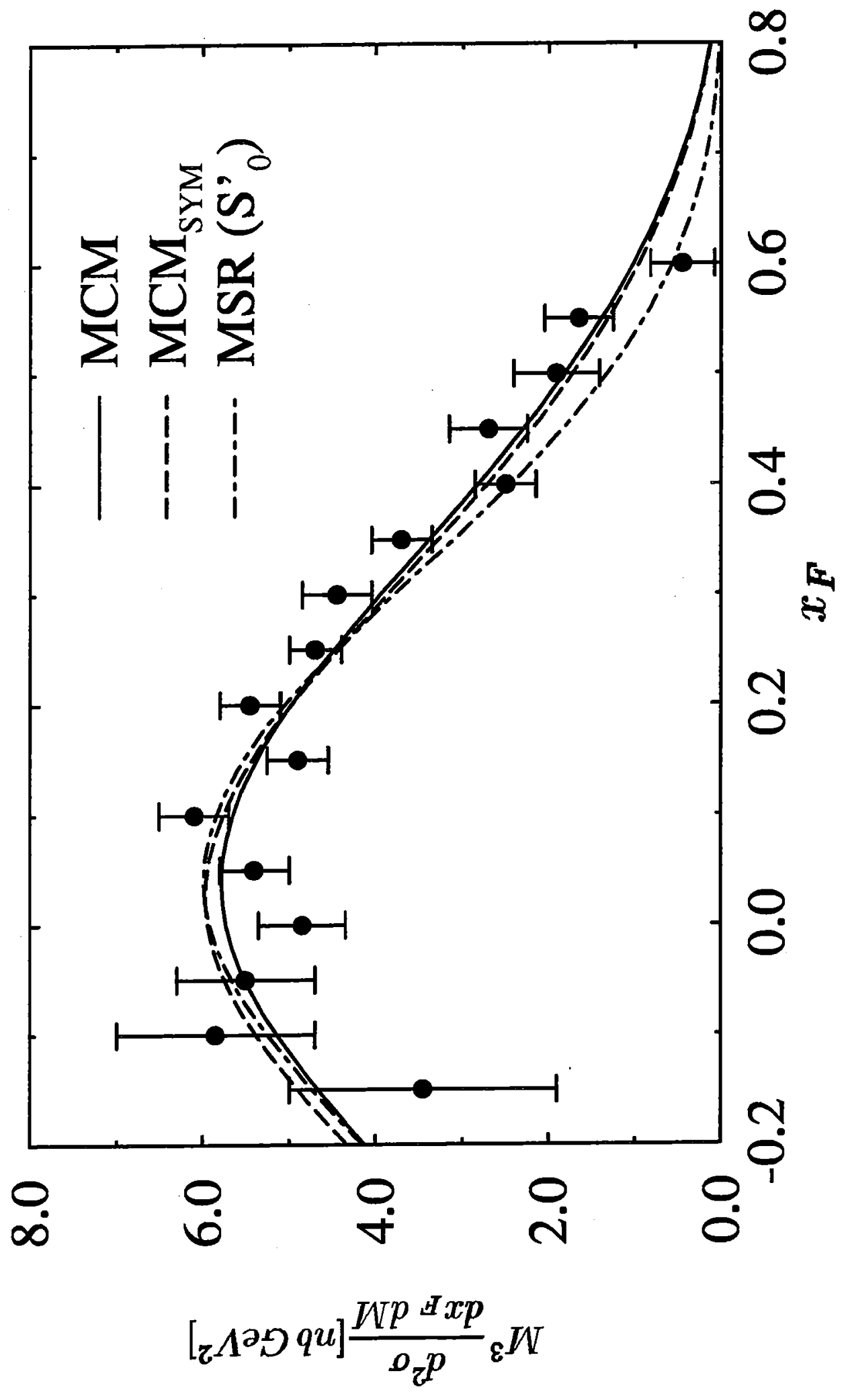

Fig. 4.9 


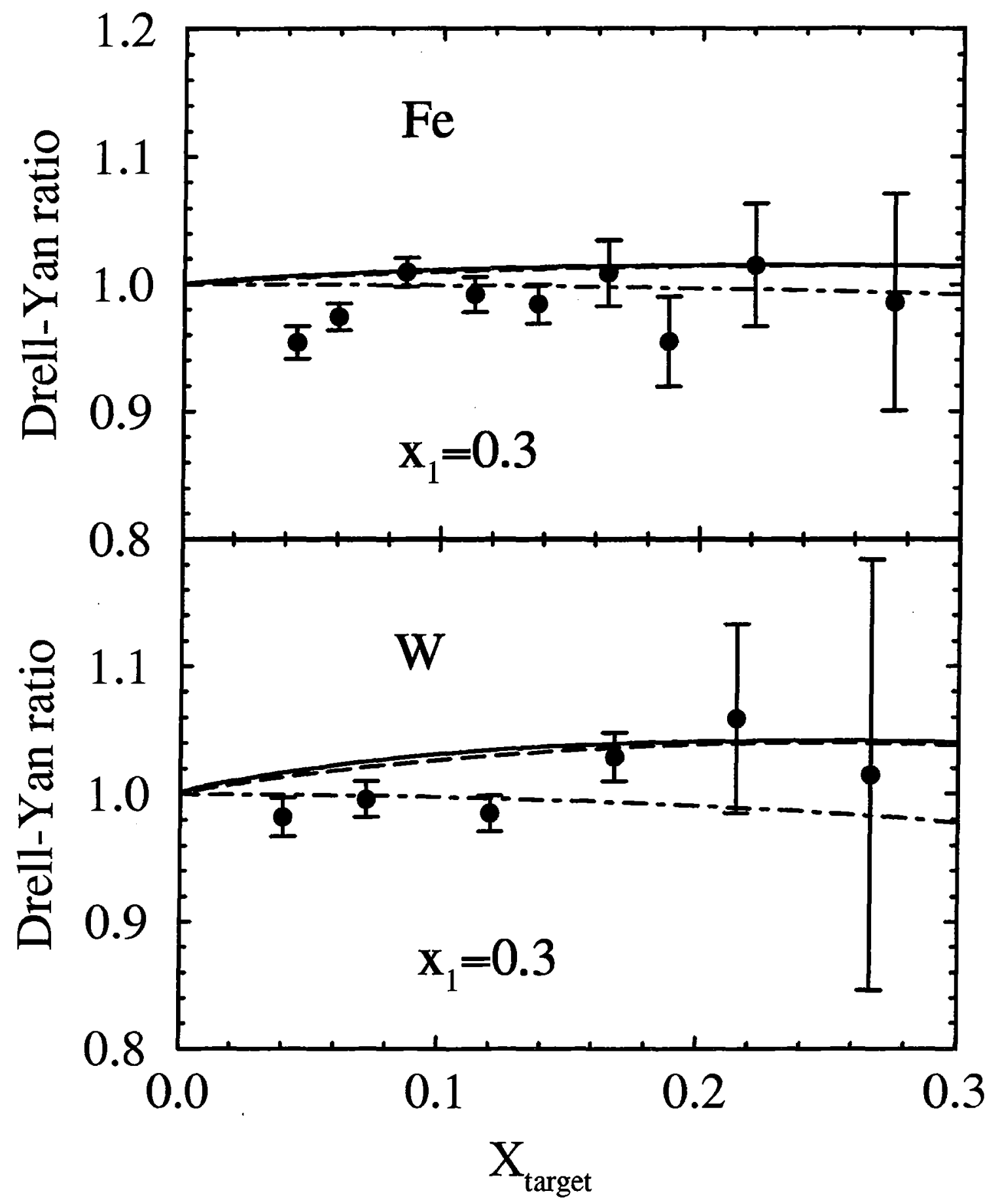

Fig. 4.10 


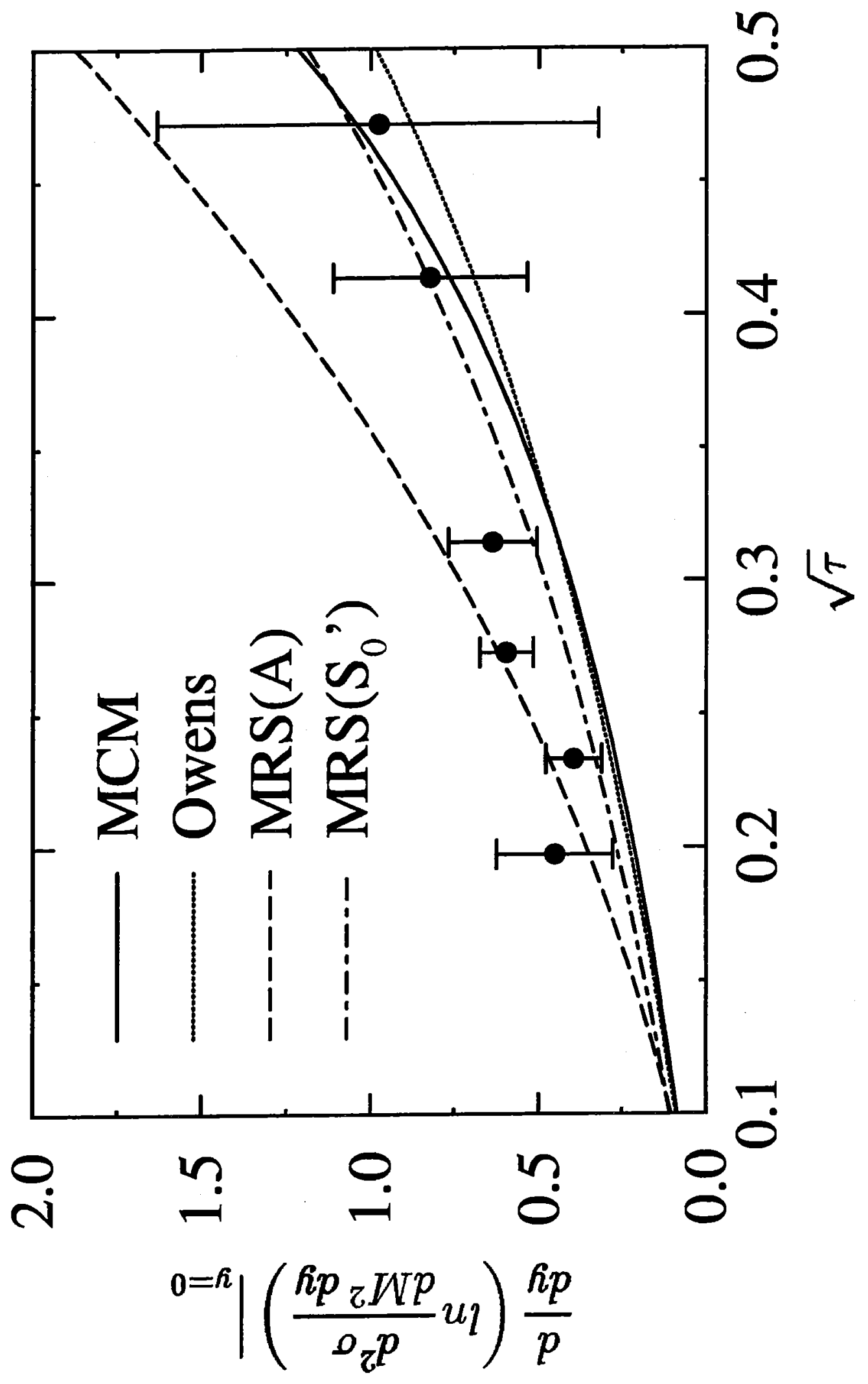

Fig. 4.11 

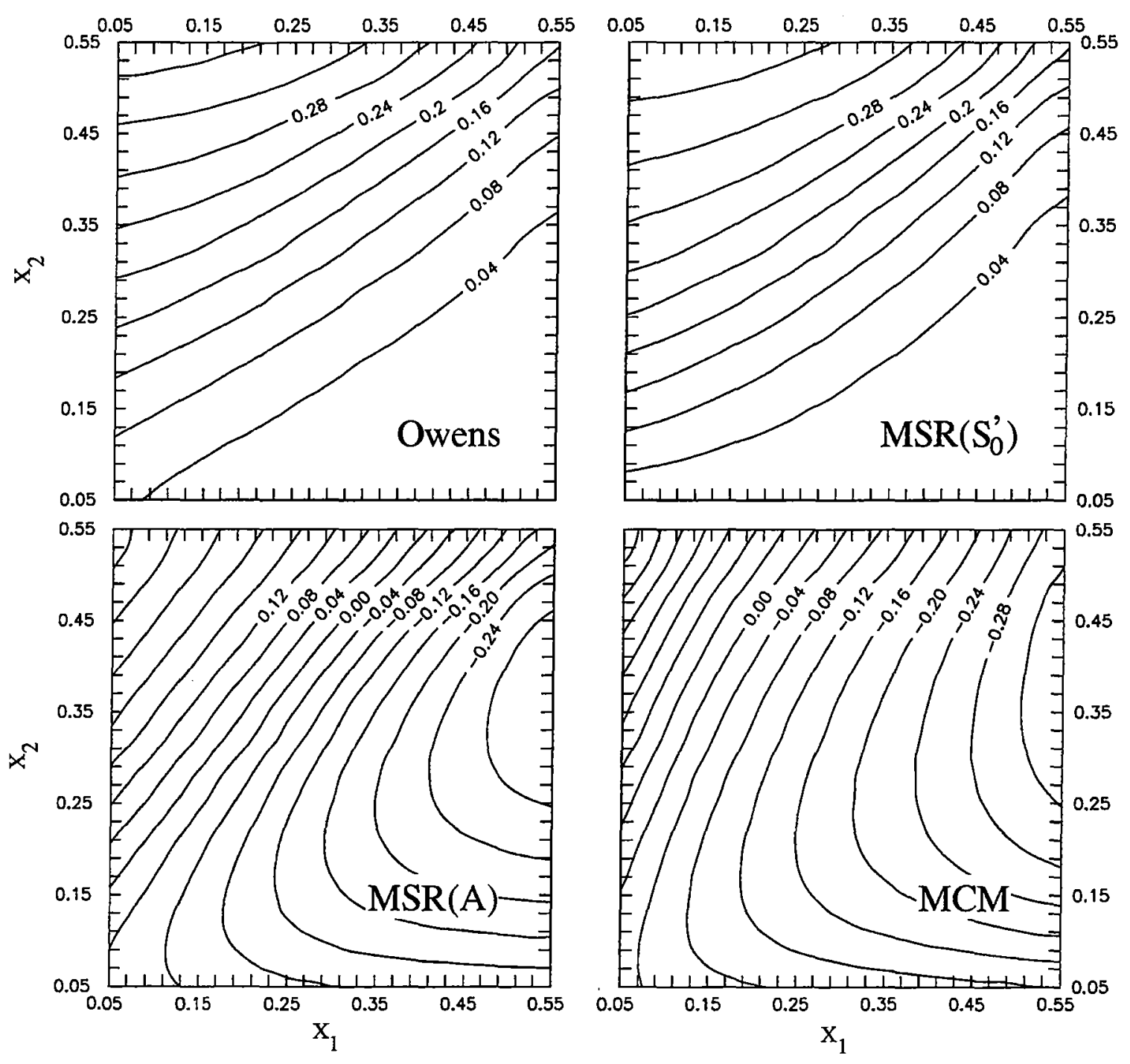

Fig. 4.12 


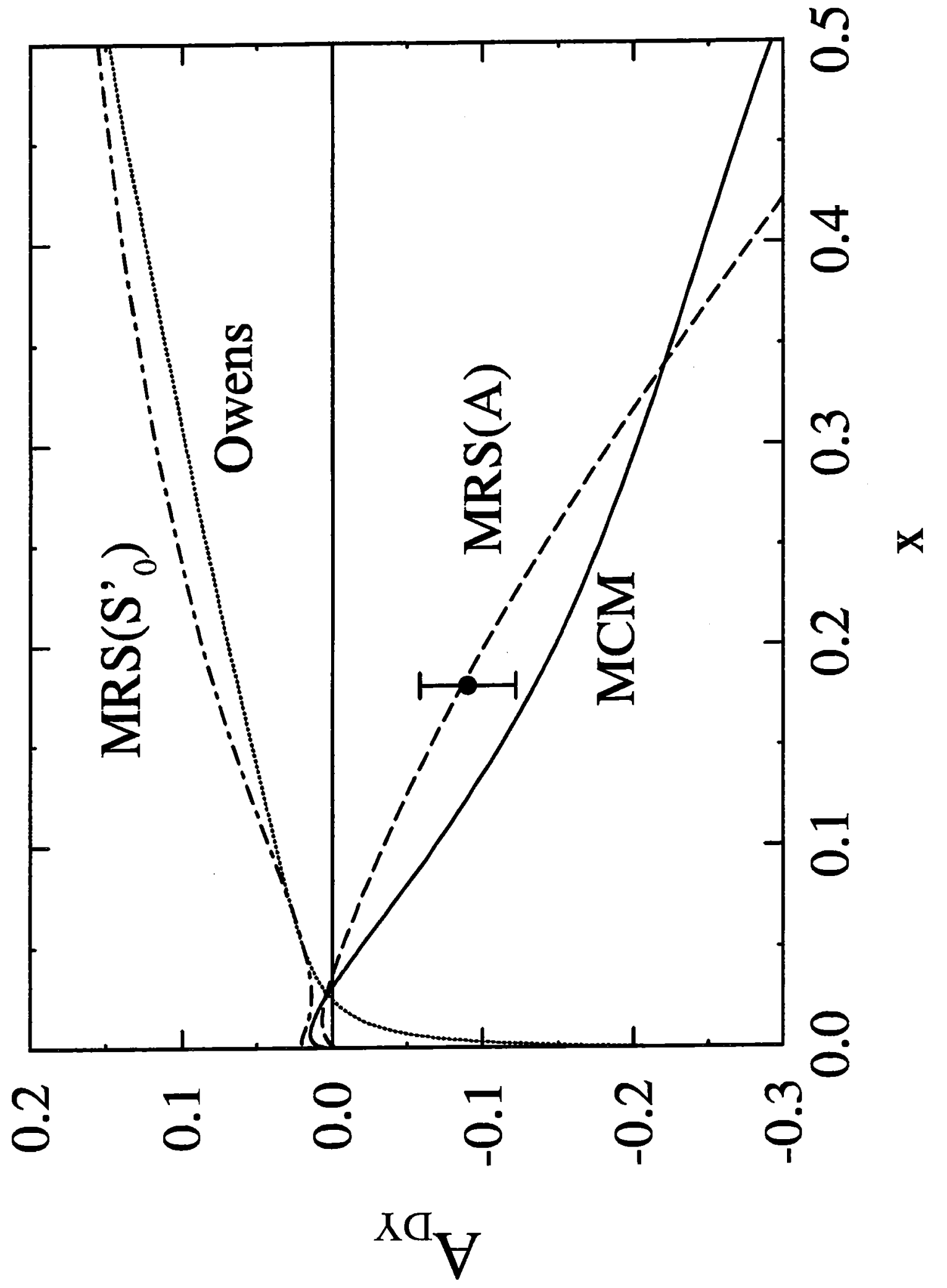

Fig. 4.13 


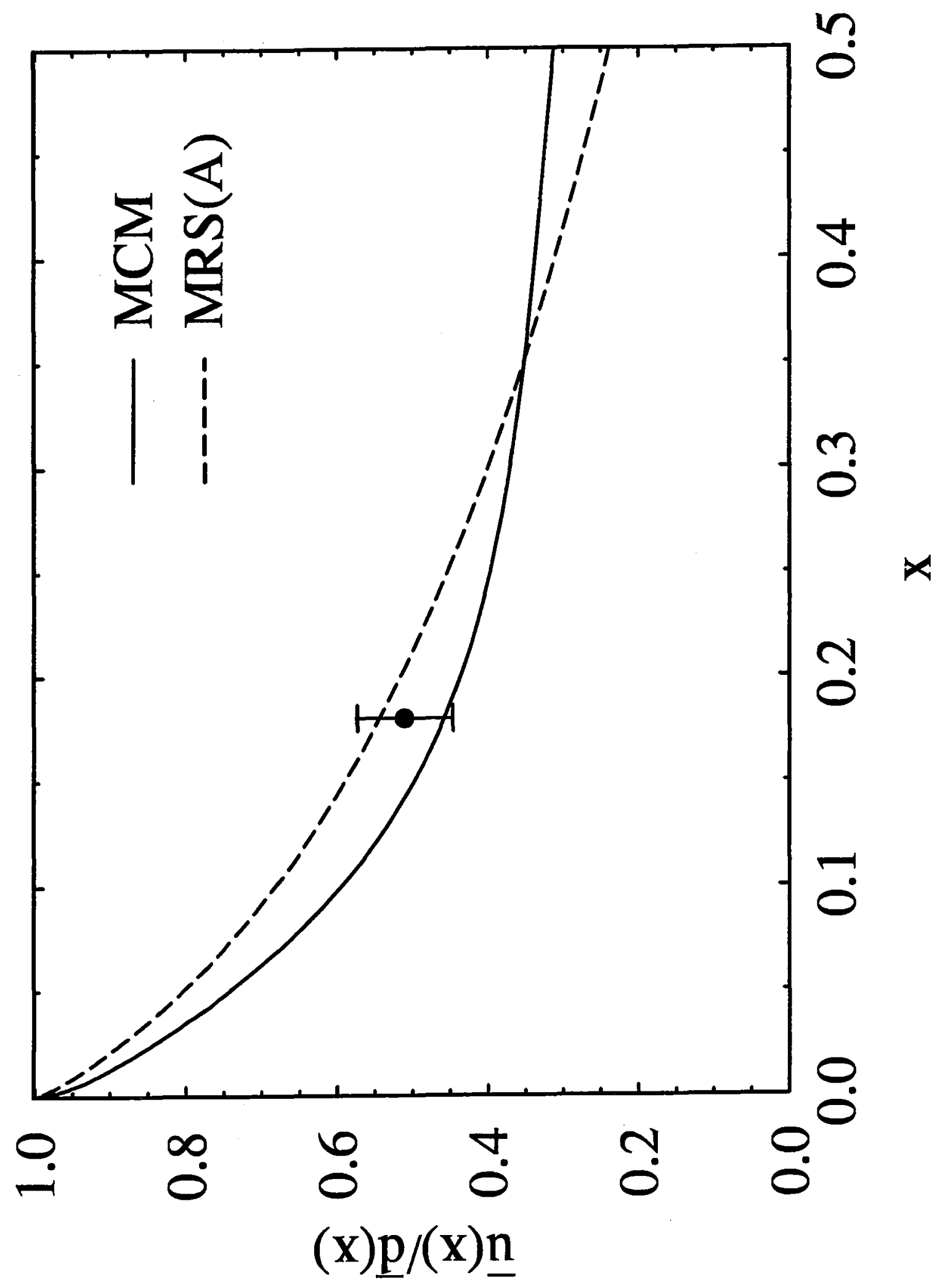

Fig. 4.14 


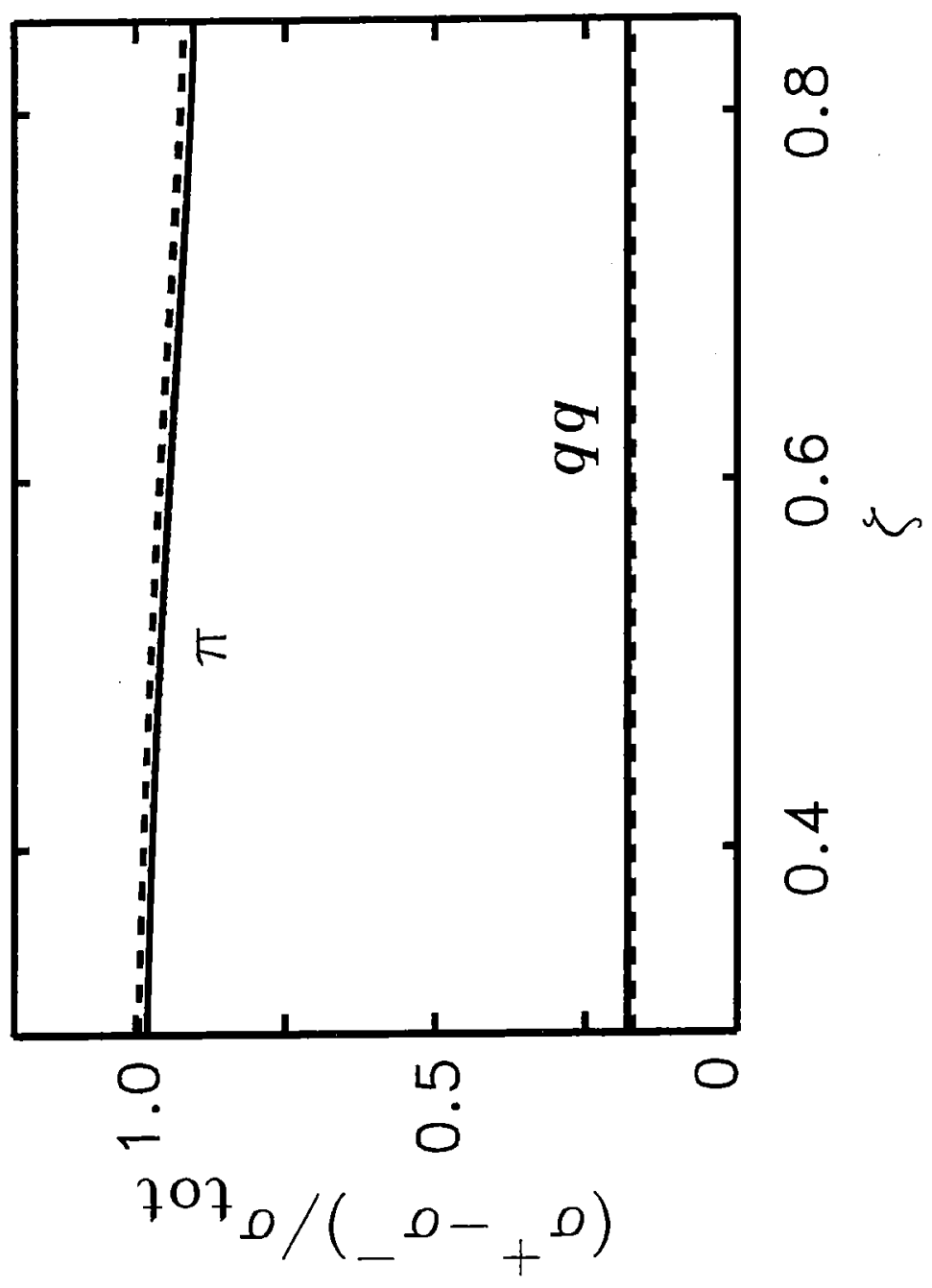

Fig. 4.15 


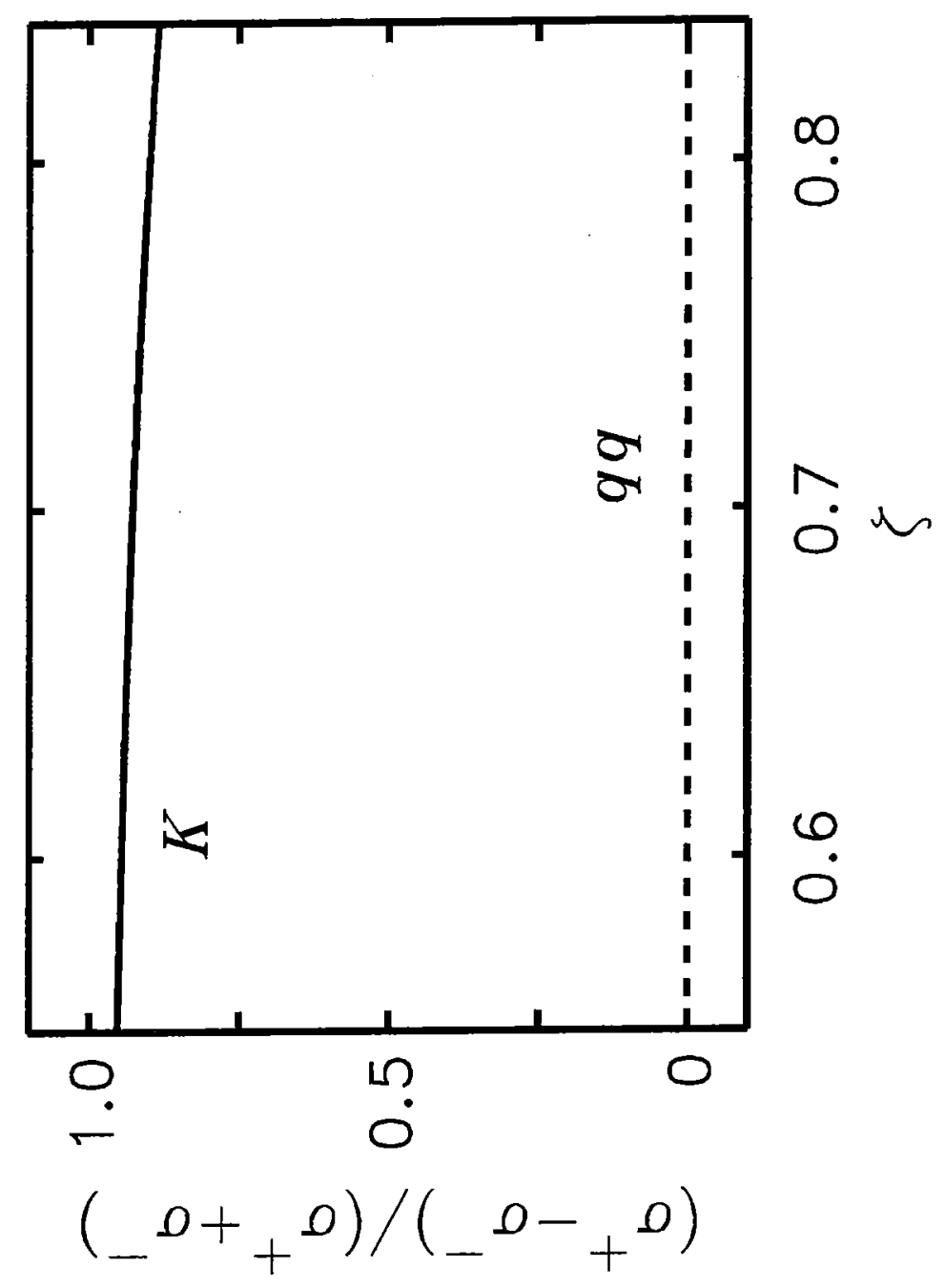

Fig. 4.16 




Fig. 4.17 

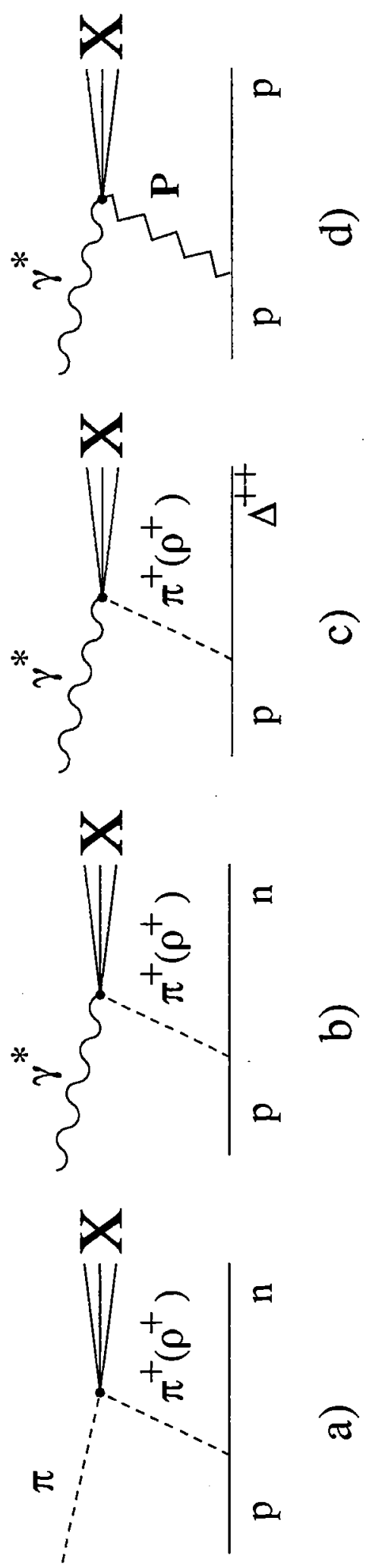

Fig. 5.1 


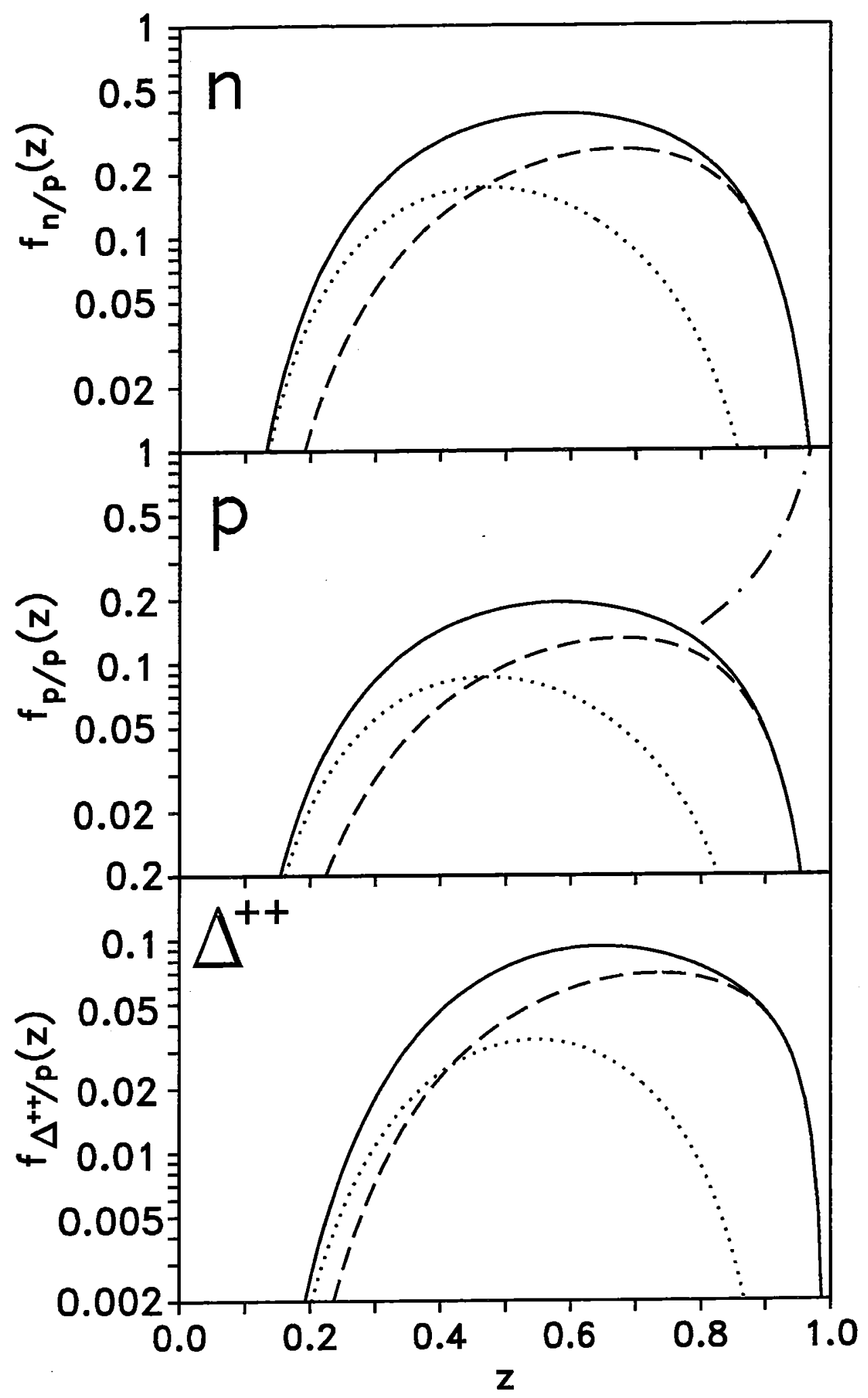

Fig. 5.2 


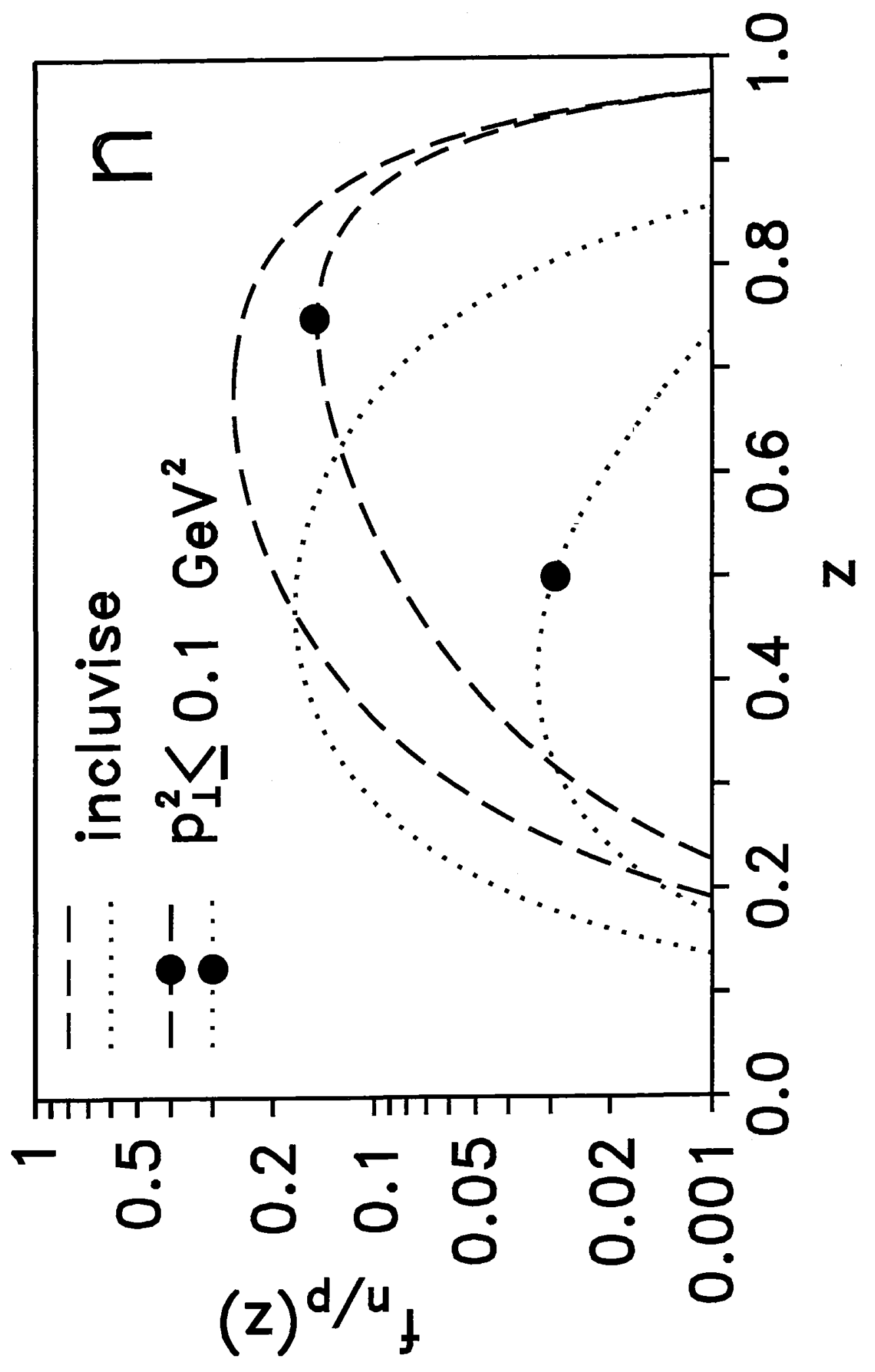

Fig. 5.3 
ISSN 0944-2952 